\title{
THE PRIMITIVE EQUATIONS OF THE ATMOSPHERE IN PRESENCE OF VAPOR SATURATION
}

\author{
MICHELE COTI ZELATI, AIMIN HUANG, IGOR KUKAVICA, \\ ROGER TEMAM, AND MOHAMMED ZIANE
}

\begin{abstract}
A modification of the classical primitive equations of the atmosphere is considered in order to take into account important phase transition phenomena due to air saturation and condensation. We provide a mathematical formulation of the problem that appears to be new in this setting, by making use of differential inclusions and variational inequalities, and which allows to develop a rather complete theory for the solutions to what turns out to be a nonlinearly coupled system of non-smooth partial differential equations. Specifically we prove global existence of quasi-strong and strong solutions, along with uniqueness results and maximum principles of physical interest.
\end{abstract}

\section{Contents}

1. Introduction

1.1. The introduction of moisture

e

1.2. A modified law of thermodynamics 4

1.3. Structure of the article 5

2. The primitive equations as differential inclusions 5

2.1. The basic model 5

2.2. The primitive equations with saturation 6

2.3. Nonlinear terms 7

2.4. Boundary and initial conditions 8

3. Mathematical setting 9

3.1. The potential temperature 9

3.2. New formulation 10

3.3. Function spaces 11

3.4. Bilinear and trilinear forms 12

3.5. Quasi-strong solutions 14

4. An approximated problem 15

4.1. Problem $\left(\mathbf{P}_{\varepsilon}\right) \quad 15$

4.2. The Galerkin approximation 16

4.3. A priori $L^{2}$-estimates (I) 17

4.4. Change of equations for $\boldsymbol{v} \quad 18$

4.5. A priori $H^{1}$-estimates for the velocity (II) 18

4.6. Local well-posedness of $\left(\mathbf{P}_{\varepsilon}\right) \quad 21$

5. Local existence of quasi-strong solutions 22

Date: April 22, 2022.

2010 Mathematics Subject Classification. 35Q35, 35B65, 35Q86, 34 A12.

Key words and phrases. Primitive equations, phase-change, saturation, global strong solutions. 
5.1. Passage to the limit as $\varepsilon \rightarrow 0$

5.2. The $(\boldsymbol{v}, T, q)$-system $\quad 25$

6. Global strong solutions 26

6.1. Strong solutions 26

6.2. Local existence of strong solutions $\quad 27$

6.3. Global existence of strong solutions 28

7. Uniqueness of strong and quasi-strong solutions 29

7.1. The moist static energy 30

7.2. Proof of Theorem 7.1 30

8. Maximum principles 35

8.1. Upper bounds for the temperature via De Giorgi iterations 36

Appendix A. Primitive equations of the atmosphere 40

$\begin{array}{ll}\text { Acknowledgments } & 47\end{array}$

References $\quad 47$

\section{INTRODUCTION}

The primitive equations (PEs) represent the classical model for the study of climate and weather prediction, describing the motion of the atmosphere when the hydrostatic assumption is enforced $[10,14,15,27,30]$. To the best of our knowledge, their mathematical study was initiated by Lions, Temam and Wang in [23-25]. This research field has quickly developed, now attracting a large number of researchers over the last two decades.

According to the classical theory of atmospheric dynamics $[10,14,15]$, for dry adiabatic motions a complete system of equations consists of the vector equation of motion

$$
\rho \frac{\mathrm{d} \mathbf{V}_{\mathbf{3}}}{\mathrm{d} t}+\rho \Omega \times \mathbf{V}_{\mathbf{3}}+\nabla_{3} p+\rho \mathbf{g}=D
$$

the equation of state

$$
p=R \rho T
$$

the equation of continuity

$$
\frac{\mathrm{d} \rho}{\mathrm{d} t}+\rho \operatorname{div}_{3} \mathbf{V}_{3}=0
$$

and the first law of thermodynamics

$$
\frac{\mathrm{d} T}{\mathrm{~d} t}-\frac{R T}{c_{p} p} \frac{\mathrm{d} p}{\mathrm{~d} t}=Q_{T}
$$

Above, the following quantities play an important role:

- $\mathbf{V}_{\mathbf{3}}=(\boldsymbol{v}, w)=$ velocity of the wind, where $\boldsymbol{v}=(u, v)$ is the horizontal velocity;

- $\rho=$ density, $p=$ pressure, $T=$ temperature;

- $\mathbf{g}=(0,0,-g)=$ the gravity, $0<c_{p}=$ specific heat, $0<R=$ specific gas constant;

- $\Omega=$ angular velocity of the earth;

- $D, Q_{T}=$ dissipation terms.

In their general three dimensional form, these equations are too complicated to be treatable both from the theoretical and the computational side. The most common physical simplification is due to the observation that the vertical dimension is usually much smaller than 
the horizontal one. Based on the hydrostatic approximation [1], this leads to the derivation of the primitive equations $[10,23-25,28,29]$. The hydrostatic assumption introduces the equation

$$
\frac{\partial p}{\partial z}=-\rho g
$$

which corresponds to the simplified form of the equation of conservation of momentum in the vertical direction. In its turn, (1.5) shows that $p$ is a decreasing function of $z$, which allows the use of $p$ as the vertical coordinate. In the $(x, y, p)$ system, the equation (1.3) now becomes

$$
\frac{\partial u}{\partial x}+\frac{\partial v}{\partial y}+\frac{\partial \omega}{\partial p}=0
$$

where $\omega$ (different from $w$ ) is now the vertical velocity of the wind in the $(x, y, p)$ system, defined by

$$
\omega=\frac{\mathrm{d} p}{\mathrm{~d} t},
$$

where the total derivative in the $(x, y, p)$-coordinates reads

$$
\frac{\mathrm{d}}{\mathrm{d} t}=\frac{\partial}{\partial t}+\boldsymbol{v} \cdot \nabla+\omega \frac{\partial}{\partial p} \text {. }
$$

The mathematical form of the equations (1.1), (1.4), and (1.6) in the $(x, y, p)$ system makes these equations very similar to the Navier-Stokes equations of incompressible fluids $[33,35,37]$ and make their mathematical theory feasible; see e.g. [23] and the review article [29] which contains a large list of mathematical references.

When moisture is included, an equation for the conservation of water must be added, which is the case in e.g. $[12,13,23,29]$. However, in these works, the equation of conservation of moisture, corresponding to the variable $q, 0 \leq q \leq 1$, is simply an equation of transport which does not account for the changes of phase, concentration/evaporation, and rain. In this work we will mainly be concerned with modifications coming from this change of model, with the aim of providing a rigorous mathematical framework for the study of such systems of equations. In earlier works $[5,6]$, two of the authors have studied the equations of the humid atmosphere with saturation by making the simplifying assumption that the velocity of the air $\boldsymbol{u}=(\boldsymbol{v}, \omega)$ is given. In this article we address the whole problem, by coupling the methods developed in $[5,6]$ with the tools developed to study the three dimensional primitive equations $[4,17-21]$.

1.1. The introduction of moisture. The equation of water vapor may be obtained in a similar manner to the derivation of the continuity equation. Calling $q$ the specific humidity, namely, the mass of water vapor in a unit mass of moist air, we can write its conservation equation $[14,15]$ as

$$
\frac{\mathrm{d} q}{\mathrm{~d} t}=\frac{S}{\rho}+D_{q}
$$

where $D_{q}$ is a suitable form of dissipation accounting for horizontal and vertical diffusion and $S$ are sources or sinks of water vapor per unit volume per unit time. This equation extends the classical conservation equation from e.g. [14, 15] by adding the dissipation 
term $D_{q}$. In general, a sink of water vapor arises from condensation or evaporation from saturated air, in which case

$$
\frac{S}{\rho}=\frac{\mathrm{d} q_{s}}{\mathrm{~d} t}
$$

where $q_{s}$ is the saturation humidity, otherwise called the saturation concentration. In general, $q_{s}$ will be either constant or a nonlinear function of the temperature $T$, and will satisfy

$$
\frac{\mathrm{d} q_{s}}{\mathrm{~d} t}=\frac{\omega}{p} F(T)
$$

for some function $F$ whose expression will be discussed in detail in Section 2.3. The common coordinate system to study the primitive equations of the atmosphere is, as we said, the $(x, y, p)$ coordinate system, where the pressure $p$ replaces the vertical coordinate $z$. Expanding equation (1.9), in view of (1.10)-(1.11) and (1.7), we obtain that $q$ undergoes the evolution equation

$$
\frac{\partial q}{\partial t}+\boldsymbol{v} \cdot \nabla q+\omega \frac{\partial q}{\partial p}=\delta \frac{\omega}{p} F(T)+D_{q}
$$

where $\delta$ is defined as

$$
\delta= \begin{cases}1, & \text { if } \omega<0 \text { and } q>q_{s} \\ 0, & \text { otherwise }\end{cases}
$$

Roughly speaking, the contribution by $F(T)$ is assumed to apply only during condensation $\left(q>q_{s}\right)$ and requires a negative $\omega$ (upward motion). Viewing $q_{s}$ as a threshold, this precisely describes the change of phase which the specific humidity $q$ obeys. The classical terminology is the following:

- $q<q_{s}$ : under-saturated regime;

- $q=q_{s}:$ saturation/condensation;

- $q>q_{s}$ : over-saturated regime.

From the point of view of partial differential equations, (1.12) introduces the mathematical difficulty of dealing with a nonlinear and discontinuous right hand side, making more challenging the proof of suitable well-posedness results.

1.2. A modified law of thermodynamics. The expression for $\mathrm{d} q_{s} / \mathrm{d} t$ in (1.11) may also be used in the thermodynamic equation

$$
-L \frac{\mathrm{d} q_{s}}{\mathrm{~d} t}=c_{p} \frac{\mathrm{d} T}{\mathrm{~d} t}-\frac{R T}{p} \frac{\mathrm{d} p}{\mathrm{~d} t},
$$

in order to properly modify the conservation equation (1.4). We then have

$$
\frac{\mathrm{d} T}{\mathrm{~d} t}-\frac{R T}{c_{p} p} \frac{\mathrm{d} p}{\mathrm{~d} t}=-\delta L \frac{\omega}{c_{p} p} F(T)+D_{T},
$$

where $\delta$ is the same as above. The first term in the right hand side of (1.13) corresponds to the energy effect of condensation or evaporation. Here, $D_{T}$ is another form of dissipation accounting, e.g. for conduction or turbulence. Written in the $(x, y, p)$ coordinate system, the above equation reads

$$
\frac{\partial T}{\partial t}+\boldsymbol{v} \cdot \nabla T+\omega \frac{\partial T}{\partial p}-\frac{R T}{c_{p} p} \omega=-\delta L \frac{\omega}{c_{p} p} F(T)+D_{T} .
$$


1.3. Structure of the article. In the next section we rephrase, following $[5,6]$, the system as a set of differential inclusions, for which we provide suitable definitions of quasistrong and strong solutions in Section 3. Section 4 is devoted to the construction of a regularized approximated problem which is useful to prove the local-in-time existence of quasi-strong solutions in Section 5. Local and global existence of strong solutions is proved in Section 6. The question of uniqueness of quasi-strong and strong solutions is addressed in Section 7, and it is very much related to the maximum principle results derived in Section 8. Finally, we conclude the article with the Appendix A, in which a modification of a result of $[20,21]$ is considered.

\section{The PRIMitive EqUATIONS AS DifFERENTIAL INCLUSIONS}

We start by defining our problem in a physical bounded domain, imposing boundary conditions and specifying the precise form of the dissipation terms appearing in (1.1), (1.12) and (1.14). The equations for temperature and specific humidity are rephrased as differential inclusions, in order to make the discontinuities in the right-hand-sides of (1.12) and (1.14) treatable from the mathematical viewpoint.

2.1. The basic model. Let $\mathcal{M}$ be a cylinder in $\mathbb{R}^{3}$ of the form

$$
\mathcal{M}=\left\{(x, y, p):(x, y) \in \mathcal{M}^{\prime}, p \in\left(p_{0}, p_{1}\right)\right\},
$$

where $\mathcal{M}^{\prime}$ is a smooth bounded domain in $\mathbb{R}^{2}$, and $p_{0}<p_{1}$ are positive constants. We denote by $\nabla, \Delta$ and div the two-dimensional gradient, Laplacian and divergence operators, respectively, that is

$$
\nabla=\left(\partial_{x}, \partial_{y}\right), \quad \Delta=\partial_{x}^{2}+\partial_{y}^{2}, \quad \operatorname{div}=\partial_{x}+\partial_{y}
$$

Analogously, the symbols $\nabla_{3}, \Delta_{3}$ and div $_{3}$ indicate their three-dimensional versions. The (viscous) primitive equations of the atmosphere read

$$
\begin{aligned}
& \frac{\partial \boldsymbol{v}}{\partial t}+(\boldsymbol{v} \cdot \nabla) \boldsymbol{v}+\omega \frac{\partial \boldsymbol{v}}{\partial p}+f \boldsymbol{k} \times \boldsymbol{v}+\nabla \Phi+\mathcal{A}_{\boldsymbol{v}} \boldsymbol{v}=\boldsymbol{S}_{\boldsymbol{v}} \\
& \frac{\partial \Phi}{\partial p}+\frac{R T}{p}=0 \\
& \operatorname{div} \boldsymbol{v}+\frac{\partial \omega}{\partial p}=0 \\
& \frac{\partial T}{\partial t}+\boldsymbol{v} \cdot \nabla T+\omega \frac{\partial T}{\partial p}-\frac{R T}{c_{p} p} \omega+\mathcal{A}_{T} T=S_{T} \\
& \frac{\partial q}{\partial t}+\boldsymbol{v} \cdot \nabla q+\omega \frac{\partial q}{\partial p}+\mathcal{A}_{q} q=S_{q} \\
& p=R \rho T .
\end{aligned}
$$

Here, $\boldsymbol{u}=(\boldsymbol{v}, \omega)$ is the three-dimensional velocity vector, $\rho, p, T$ are the density, pressure and the temperature distribution, and $q$ is the specific humidity, measuring the amount of vapor in the air. In (2.1), $f$ is the Coriolis force parameter and $\boldsymbol{k}$ is the unit vector in the direction of the poles (from south to north). As the equations we consider here are the viscous PEs of the atmosphere, the symbols $\mathcal{A}_{\boldsymbol{v}}, \mathcal{A}_{T}$ and $\mathcal{A}_{q}$ denote diffusion operators, 
with suitable eddy viscosity coefficients:

$$
\mathcal{A}_{\star}=-\mu_{\star} \Delta-\nu_{\star} \frac{\partial}{\partial p}\left(\left(\frac{g p}{R \bar{T}}\right)^{2} \frac{\partial}{\partial p}\right),
$$

where $\star$ can either be $\boldsymbol{v}, T$, or $q$, and $\bar{T}=\bar{T}(p)$ is a given average temperature over the isobar with pressure $p$, for which we assume the existence of two positive constants $\bar{T}_{*}$ and $\bar{T}^{*}$ such that

$$
\bar{T}_{*} \leq \bar{T}(p) \leq \bar{T}^{*}
$$

Concerning the right hand sides, $S_{T}$ corresponds to the sum of the heating of the sun and the heat added or removed by condensation or evaporation; $S_{q}$ represents the amount of water added or removed by condensation or evaporation. Finally, $\boldsymbol{S}_{\boldsymbol{v}}$, which vanishes in reality, is a forcing term usually added for mathematical generality and to possibly handle nonhomogenous boundary conditions.

2.2. The primitive equations with saturation. When studying the climate dynamics around the equator, the humidity equation, describing the ratio of vapor in the air, becomes very important and it is necessary to account for the possible saturation of vapor leading to condensation (clouds) and rain. In the recent works [5,6], the authors considered the coupling of the humidity equation and the temperature equation with a given velocity vector field $\boldsymbol{u}=(\boldsymbol{v}, \omega)$. These two differential inclusions replacing (2.4) and (2.5) read (see $[14,15])$ :

$$
\begin{aligned}
& \frac{\partial T}{\partial t}+\boldsymbol{v} \cdot \nabla T+\omega \frac{\partial T}{\partial p}-\frac{R}{c_{p} p} \omega T+\mathcal{A}_{T} T \in \frac{L}{c_{p} p} \omega^{-} H\left(q-q_{s}\right) F(T)+S_{T}, \\
& \frac{\partial q}{\partial t}+\boldsymbol{v} \cdot \nabla q+\omega \frac{\partial q}{\partial p}+\mathcal{A}_{q} q \in-\frac{1}{p} \omega^{-} H\left(q-q_{s}\right) F(T)+S_{q} .
\end{aligned}
$$

Here $\omega^{-}=\max \{-\omega, 0\}$ is the negative part of $\omega$, and $H\left(q-q_{s}\right)$ is the Heaviside multivalued function, i.e.,

$$
H(r)= \begin{cases}0, & r<0 \\ {[0,1],} & r=0 \\ 1, & r>0\end{cases}
$$

The papers $[5,6]$ provide the existence, uniqueness, and maximum principles of weak solutions to the equations (2.8)-(2.9), with the velocity $\boldsymbol{u}$ given in some suitable Sobolev spaces.

In this article, we consider the full nonlinear PEs in the presence of vapor saturation. Specifically, we replace equations (2.4)-(2.5) with the differential inclusions (2.8)-(2.9) in order to take the saturation phenomenon into account. More precisely, the full system 
under study reads now

$$
\begin{aligned}
& \frac{\partial \boldsymbol{v}}{\partial t}+(\boldsymbol{v} \cdot \nabla) \boldsymbol{v}+\omega \frac{\partial \boldsymbol{v}}{\partial p}+f \boldsymbol{k} \times \boldsymbol{v}+\nabla \Phi+\mathcal{A}_{\boldsymbol{v}} \boldsymbol{v}=\boldsymbol{S}_{\boldsymbol{v}}, \\
& \frac{\partial \Phi}{\partial p}+\frac{R T}{p}=0 \\
& \operatorname{div} \boldsymbol{v}+\frac{\partial \omega}{\partial p}=0, \\
& \frac{\partial T}{\partial t}+\boldsymbol{v} \cdot \nabla T+\omega \frac{\partial T}{\partial p}-\frac{R}{c_{p} p} \omega T+\mathcal{A}_{T} T \in \frac{L}{c_{p} p} \omega^{-} H\left(q-q_{s}\right) F(T)+S_{T}, \\
& \frac{\partial q}{\partial t}+\boldsymbol{v} \cdot \nabla q+\omega \frac{\partial q}{\partial p}+\mathcal{A}_{q} q \in-\frac{1}{p} \omega^{-} H\left(q-q_{s}\right) F(T)+S_{q}, \\
& p=R \rho T .
\end{aligned}
$$

Our aim here is to study the coupled system (2.11)-(2.16) and prove the existence and uniqueness of the global (quasi)-strong solutions defined in Sections 3.5 and 6.1, for a constant saturation concentration $q_{s} \in(0,1)$.

2.3. Nonlinear terms. An important difference between our system and the one considered in the classical references $[15,28]$ (see also [23,26]) are the nonlinear terms. Firstly, the temperature equation (2.14) involves the nonlinear (and possibly anti-dissipative) term

$$
-\frac{R}{c_{p} p} \omega T
$$

on the left hand side. This requires some care from the very beginning, as shown in [9].

Secondly, in (2.14) and (2.15), the nonlinearity $F: \mathbb{R} \rightarrow \mathbb{R}$ is obtained by setting [14,32]

$$
F(\xi)=q_{s} \xi\left(\frac{L R-c_{p} R_{v} \xi}{c_{p} R_{v} \xi^{2}+q_{s} L^{2}}\right)
$$

where $c_{p}, L$ and $R$ are the positive constant described above, and $R_{v}$ is equal to the gas constant for water vapor. By a direct calculation, we see that $F$ is a globally Lipschitz bounded function, namely

$$
\left|F\left(\xi_{1}\right)-F\left(\xi_{2}\right)\right| \leq c_{F}\left|\xi_{1}-\xi_{2}\right|, \quad \forall \xi_{1}, \xi_{2} \in \mathbb{R},
$$

and

$$
|F(\xi)| \leq C_{F}, \quad \forall \xi \in \mathbb{R} .
$$

Since $F(0)=0$, we also obtain from (2.18) that

$$
|F(\xi)| \leq c_{F}|\xi|, \quad \forall \xi \in \mathbb{R} .
$$

Moreover,

$$
F\left(\xi_{0}\right)=0 \quad \text { for } \quad \xi_{0}=\frac{L R}{c_{p} R_{v}}
$$

Therefore,

$$
F(\xi) \geq 0 \quad \Leftrightarrow \quad \xi \in\left[0, \xi_{0}\right] .
$$


2.4. Boundary and initial conditions. The boundary of $\mathcal{M}$ is partitioned into three parts as $\partial \mathcal{M}=\Gamma_{i} \cup \Gamma_{b} \cup \Gamma_{\ell}$, where

$$
\begin{aligned}
& \Gamma_{i}=\left\{(x, y, p) \in \overline{\mathcal{M}}: p=p_{1}\right\}, \\
& \Gamma_{u}=\left\{(x, y, p) \in \overline{\mathcal{M}}: p=p_{0}\right\}, \\
& \Gamma_{\ell}=\left\{(x, y, p) \in \overline{\mathcal{M}}:(x, y) \in \partial \mathcal{M}^{\prime}, p_{0} \leq p \leq p_{1}\right\} .
\end{aligned}
$$

We supplement system (2.11)-(2.16) with the following physically relevant (homogeneous) boundary conditions: wind-driven on the top surface and free-slip and non-heat flux on the side walls and the bottom (see e.g. $[4,6]$ ):

$$
\begin{aligned}
& \text { on } \Gamma_{i}: \quad \frac{\partial \boldsymbol{v}}{\partial p}=-\frac{\alpha_{\boldsymbol{v}}}{\nu_{\boldsymbol{v}}} \boldsymbol{v}, \quad \omega=0, \quad \frac{\partial T}{\partial p}=-\frac{\alpha_{T}}{\nu_{T}} T, \quad \frac{\partial q}{\partial p}=-\frac{\alpha_{q}}{\nu_{q}} q ; \\
& \text { on } \Gamma_{u}: \frac{\partial \boldsymbol{v}}{\partial p}=0, \quad \omega=0, \quad \frac{\partial T}{\partial p}=0, \quad \frac{\partial q}{\partial p}=0 \text {; } \\
& \text { on } \Gamma_{\ell}: \quad \boldsymbol{v} \cdot \boldsymbol{n}=0, \quad \frac{\partial(\boldsymbol{v} \cdot \boldsymbol{\tau})}{\partial \boldsymbol{n}}=0, \quad \frac{\partial T}{\partial \boldsymbol{n}}=0, \quad \frac{\partial q}{\partial \boldsymbol{n}}=0,
\end{aligned}
$$

where $\boldsymbol{n}$ and $\boldsymbol{\tau}$ are the unit normal and tangent vectors to $\Gamma_{\ell}$ respectively and $\alpha_{T}, \alpha_{q}>0$ are given positive constants. In addition, we supplement system (2.11)-(2.16) with the initial conditions

$$
\begin{aligned}
& \boldsymbol{v}(x, y, p, 0)=\boldsymbol{v}_{0}(x, y, p) \\
& T(x, y, p, 0)=T_{0}(x, y, p) \\
& q(x, y, p, 0)=q_{0}(x, y, p) .
\end{aligned}
$$

Remark 2.1. In limited area atmospheric models, the free-slip boundary conditions for the lateral boundary in (2.26) are more appropriate to avoid artificial boundary layer (see e.g. [39]). We could also consider the no-slip boundary conditions or periodic boundary conditions for the lateral boundary and the main results in this article still hold for these boundary conditions.

Remark 2.2. A relevant non-homogeneous version of the above boundary conditions (2.24) $-(2.26)$ can be be written as follows:

$$
\text { on } \Gamma_{i}: \frac{\partial \boldsymbol{v}}{\partial p}=\frac{\alpha_{\boldsymbol{v}}}{\nu_{\boldsymbol{v}}}\left(\left(p_{1}-p_{0}\right) \boldsymbol{v}_{*}-\boldsymbol{v}\right), \quad \omega=0, \quad \frac{\partial T}{\partial p}=\frac{\alpha_{T}}{\nu_{T}}\left(T_{*}-T\right), \quad \frac{\partial q}{\partial p}=\frac{\alpha_{q}}{\nu_{q}}\left(q_{*}-q\right) ;
$$

$$
\text { on } \Gamma_{u}: \frac{\partial \boldsymbol{v}}{\partial p}=0, \quad \omega=0, \quad \frac{\partial T}{\partial p}=0, \quad \frac{\partial q}{\partial p}=0 ;
$$

$$
\text { on } \Gamma_{\ell}: \boldsymbol{v} \cdot \boldsymbol{n}=0, \quad \frac{\partial(\boldsymbol{v} \cdot \boldsymbol{\tau})}{\partial \boldsymbol{n}}=0, \quad \frac{\partial T}{\partial \boldsymbol{n}}=0, \quad \frac{\partial q}{\partial \boldsymbol{n}}=0 .
$$

In this setting, $\boldsymbol{v}_{*}(x, y)$ is the wind stress on the ocean surface and $T_{*}(x, y)$ and $q_{*}(x, y)$ are typical temperature and specific humidity distributions at the bottom surface of the atmosphere, respectively. Due to the boundary conditions (2.28)-(2.30), it is natural to 
assume that $\boldsymbol{v}_{*}, T_{*}$ and $q_{*}$ satisfy the boundary compatibility conditions

$$
\begin{aligned}
& \boldsymbol{v}_{*} \cdot \boldsymbol{n}=0, \quad \frac{\partial\left(\boldsymbol{v}_{*} \cdot \boldsymbol{\tau}\right)}{\partial \boldsymbol{n}}=0, \quad \text { on } \partial \mathcal{M}^{\prime}, \\
& \frac{\partial T_{*}}{\partial \boldsymbol{n}}=0, \quad \frac{\partial q_{*}}{\partial \boldsymbol{n}}=0, \quad \text { on } \partial \mathcal{M}^{\prime} .
\end{aligned}
$$

As observed in [4, Remark 1], if we make the following variables change

$$
\left\{\begin{array}{l}
\tilde{\boldsymbol{v}}=\boldsymbol{v}-\left(p_{1}-p_{0}\right) \boldsymbol{v}_{*}, \\
\widetilde{T}=T-T_{*} \\
\tilde{q}=q-q_{*}
\end{array}\right.
$$

then $(\tilde{\boldsymbol{v}}, \widetilde{T}, \tilde{q})$ satisfies the homogeneous boundary conditions $(2.24)-(2.26)$ thanks to the compatibility boundary conditions $(2.31)$, and the extra terms involving $\left(\boldsymbol{v}_{*}, T_{*}, q_{*}\right)$ appearing in the new set of equations similar to those (2.11)-(2.16) are lower order terms and easy to handle. Hence for simplicity and without loss of generality we assume that $\boldsymbol{v}_{*}=T_{*}=q_{*}=0$, corresponding to our choice (2.24)-(2.26). Therefore, the results presented here are still valid for general $\left(\boldsymbol{v}_{*}, T_{*}, q_{*}\right)$ provided these quantities are smooth enough.

\section{Mathematical SETting}

The weak formulation of the system (2.11)-(2.16) along with its boundary condition requires the introduction of a rather large set of functional analytic tools, including a variational inequality to represent the Heaviside graph as the subdifferential of a convex functional. This section is therefore devoted to making more precise the mathematical formulation of the equations under study.

3.1. The potential temperature. In order to eliminate the demanding nonlinear term

$$
\frac{R}{p} \omega T
$$

in the temperature equation (2.14), we introduce the so-called potential temperature

$$
\theta=T\left(\frac{p_{0}}{p}\right)^{R / c_{p}}
$$

Using $\omega=\mathrm{d} p / \mathrm{d} t$ where $\mathrm{d} / \mathrm{d} t$ is defined in (1.8), a direct computation shows that

$$
\frac{\mathrm{d} T}{\mathrm{~d} t}-\frac{R \omega}{c_{p} p} T=\left(\frac{p}{p_{0}}\right)^{R / c_{p}} \frac{\mathrm{d} \theta}{\mathrm{d} t} .
$$

In this way, the equation (2.14) becomes

$$
\frac{\partial \theta}{\partial t}+\boldsymbol{v} \cdot \nabla \theta+\omega \frac{\partial \theta}{\partial p}+\mathcal{A}_{\theta} \theta \in \frac{L}{c_{p} p}\left(\frac{p_{0}}{p}\right)^{R / c_{p}} \omega^{-} H\left(q-q_{s}\right) \widetilde{F}(p, \theta)+S_{\theta},
$$

where the operator $\mathcal{A}_{T}$ is replaced by

$$
\mathcal{A}_{\theta}=-\mu_{T} \Delta-\nu_{T}\left(\frac{p_{0}}{p}\right)^{R / c_{p}} \frac{\partial}{\partial p}\left(\frac{g p}{R \bar{T}}\right)^{2} \frac{\partial}{\partial p}\left(\frac{p}{p_{0}}\right)^{R / c_{p}},
$$


and the nonlinear term $F$ takes the form

$$
\widetilde{F}(p, \theta)=F\left[\left(\frac{p}{p_{0}}\right)^{R / c_{p}} \theta\right]
$$

Note that, thanks to the properties (2.18)-(2.20) of $F$ and the fact that $p \in\left[p_{0}, p_{1}\right]$, we have

$$
|\widetilde{F}(p, \xi)| \leq C_{\widetilde{F}}, \quad\left|\widetilde{F}\left(p, \xi_{1}\right)-\widetilde{F}\left(p, \xi_{2}\right)\right| \leq c_{\widetilde{F}}\left|\xi_{1}-\xi_{2}\right|, \quad|\widetilde{F}(p, \xi)| \leq c_{\widetilde{F}}|\xi|,
$$

where the last inequality follows from the fact that $\widetilde{F}(p, 0)=F(0)=0$ for every $p$. In the same way, the boundary conditions become

$$
\left.\left(\nu_{T} \frac{\partial \theta}{\partial p}+\alpha_{\theta} \theta\right)\right|_{\Gamma_{i}}=0,\left.\quad\left(\frac{\partial \theta}{\partial p}+\frac{R}{c_{p} p_{0}} \theta\right)\right|_{\Gamma_{u}}=0,\left.\quad \frac{\partial \theta}{\partial \boldsymbol{n}}\right|_{\Gamma_{\ell}}=0,
$$

where

$$
\alpha_{\theta}=\alpha_{T}+\nu_{T} \frac{R}{c_{p} p_{1}}
$$

and the initial condition turns into

$$
\theta(x, y, p, 0)=\theta_{0}(x, y, p)=T_{0}(x, y, p)\left(\frac{p_{0}}{p}\right)^{R / c_{p}} .
$$

Remark 3.1. It is clear that, since $0<p_{0} \leq p \leq p_{1}$, the properties that we will derive for $\theta$ will be translated into analogous properties for the temperature $T$.

3.2. New formulation. Integrating (2.12) in the $p$-direction gives

$$
\begin{aligned}
\Phi(x, y, p, t) & =\Phi_{s}(x, y, t)+\int_{p}^{p_{1}} \frac{R}{p^{\prime}} T\left(x, y, p^{\prime}, t\right) \mathrm{d} p^{\prime} \\
& =\Phi_{s}(x, y, t)+\int_{p}^{p_{1}} \frac{R}{p^{\prime}}\left(\frac{p^{\prime}}{p_{0}}\right)^{R / c_{p}} \theta\left(x, y, p^{\prime}, t\right) \mathrm{d} p^{\prime},
\end{aligned}
$$

where $\Phi_{s}=\Phi_{s}(x, y, t)$ is the pressure at the bottom of the atmosphere when $p=p_{1}$. In the same manner, using the boundary conditions (2.25), we infer from (2.13) that $\omega=\omega(\boldsymbol{v})$ satisfies

$$
\omega(x, y, p, t)=\int_{p}^{p_{1}} \nabla \cdot \boldsymbol{v}\left(x, y, p^{\prime}, t\right) \mathrm{d} p^{\prime},
$$

and the following constraint must be satisfied:

$$
\int_{p_{0}}^{p_{1}} \nabla \cdot \boldsymbol{v}(x, y, p, t) \mathrm{d} p=\nabla \cdot \int_{p_{0}}^{p_{1}} \boldsymbol{v}(x, y, p, t) \mathrm{d} p=0 .
$$

We aim to write the fully nonlinear PEs in the prognostic variables, namely in $(\boldsymbol{v}, \theta, q)$. The other variables $T, \rho, \Phi, \omega$ can be determined by (2.16), (3.1), (3.7), and (3.8), and they are called the diagnostic variables. Defining

$$
D(\omega, \theta, q)=\frac{1}{p} \omega^{-} H\left(q-q_{s}\right) \widetilde{F}(p, \theta)
$$


the PEs in the prognostic variables $(\boldsymbol{v}, \theta, q)$ read

$$
\begin{aligned}
& \frac{\partial \boldsymbol{v}}{\partial t}+\boldsymbol{v} \cdot \nabla \boldsymbol{v}+\omega \frac{\partial \boldsymbol{v}}{\partial p}+f \boldsymbol{k} \times \boldsymbol{v}+\nabla \Phi_{s}+\nabla \int_{p}^{p_{1}} \frac{R}{p^{\prime}}\left(\frac{p^{\prime}}{p_{0}}\right)^{R / c_{p}} \theta \mathrm{d} p^{\prime}+\mathcal{A}_{\boldsymbol{v}} \boldsymbol{v}=\boldsymbol{S}_{\boldsymbol{v}} \\
& \frac{\partial \theta}{\partial t}+\boldsymbol{v} \cdot \nabla \theta+\omega \frac{\partial \theta}{\partial p}+\mathcal{A}_{\theta} \theta \in \frac{L}{c_{p}}\left(\frac{p_{0}}{p}\right)^{R / c_{p}} D(\omega, \theta, q)+S_{\theta} \\
& \frac{\partial q}{\partial t}+\boldsymbol{v} \cdot \nabla q+\omega \frac{\partial q}{\partial p}+\mathcal{A}_{q} q \in-D(\omega, \theta, q)+S_{q}
\end{aligned}
$$

with the boundary conditions

$$
\left.\left(\nu_{\boldsymbol{v}} \frac{\partial \boldsymbol{v}}{\partial p}+\alpha_{\boldsymbol{v}} \boldsymbol{v}\right)\right|_{\Gamma_{i}}=0,\left.\quad \frac{\partial \boldsymbol{v}}{\partial p}\right|_{\Gamma_{u}}=0,\left.\quad \boldsymbol{v} \cdot \boldsymbol{n}\right|_{\Gamma_{\ell}}=\left.\frac{\partial(\boldsymbol{v} \cdot \boldsymbol{\tau})}{\partial \boldsymbol{n}}\right|_{\Gamma_{\ell}}=0
$$

$$
\left.\left(\nu_{T} \frac{\partial \theta}{\partial p}+\alpha_{\theta} \theta\right)\right|_{\Gamma_{i}}=0,\left.\quad\left(\frac{\partial \theta}{\partial p}+\frac{R}{c_{p} p_{0}} \theta\right)\right|_{\Gamma_{u}}=0,\left.\quad \frac{\partial \theta}{\partial \boldsymbol{n}}\right|_{\Gamma_{\ell}}=0,
$$

$$
\left.\left(\nu_{q} \frac{\partial q}{\partial p}+\alpha_{q} q\right)\right|_{\Gamma_{i}}=0,\left.\quad \frac{\partial q}{\partial p}\right|_{\Gamma_{u}}=0,\left.\quad \frac{\partial q}{\partial \boldsymbol{n}}\right|_{\Gamma_{\ell}}=0,
$$

and the initial conditions

$$
\begin{aligned}
\boldsymbol{v}(x, y, p, 0) & =\boldsymbol{v}_{0}(x, y, p), \\
\theta(x, y, p, 0) & =\theta_{0}(x, y, p), \\
q(x, y, p, 0) & =q_{0}(x, y, p) .
\end{aligned}
$$

3.3. Function spaces. Here and throughout this article, we will not distinguish the notations for vector and scalar function spaces whenever they are self-evident from the context. Denote by $H^{s}=H^{s}(\mathcal{M})$ the classical Sobolev spaces of order $s$ on $\mathcal{M}$, and by $L^{p}=L^{p}(\mathcal{M})(1 \leq p \leq \infty)$ the classical $L^{p}$-Lebesgue space with norm $\|\cdot\|_{L^{p}}$. The only exceptions will be made for the space $L^{2}$, whose norm will be written with the single bar $|\cdot|$ and the scalar product as $(\cdot, \cdot)$, and for the space $H^{1}$, whose scalar product is defined as

$$
((\varphi, \tilde{\varphi}))=(\nabla \varphi, \nabla \tilde{\varphi})+\int_{\mathcal{M}}\left(\frac{g p}{R \bar{T}}\right)^{2} \partial_{p} \varphi \partial_{p} \tilde{\varphi} \mathrm{d} \mathcal{M}+\int_{\Gamma_{i}}\left(\frac{g p_{1}}{R \bar{T}}\right)^{2} \varphi \tilde{\varphi} \mathrm{d} \Gamma_{i} .
$$

and its norm denoted by

$$
\|\varphi\|=((\varphi, \varphi))^{1 / 2}
$$

Using the generalized Poincaré inequality (see e.g. [36, pp. 49-50]), the norm $\|\cdot\|$ is equivalent to the $H^{1}$-norm. Regarding the velocity field $\boldsymbol{v}$, we introduce the space

$$
\mathcal{V}=\left\{\boldsymbol{v} \in C^{\infty}\left(\mathcal{M} ; \mathbb{R}^{2}\right): \nabla \cdot \int_{p_{0}}^{p_{1}} \boldsymbol{v}\left(x, y, p^{\prime}\right) \mathrm{d} p^{\prime}=0, \boldsymbol{v} \text { satisfies }(3.14)\right\}
$$

along with the $L^{2}$ and $H^{1}$-like spaces

$$
\begin{aligned}
& \mathbf{H}=\text { The closure of } \mathcal{V} \text { with respect to the norm of }\left(L^{2}\right)^{2}, \\
& \mathbf{V}=\text { The closure of } \mathcal{V} \text { with respect to the norm of }\left(H^{1}\right)^{2} .
\end{aligned}
$$


Due to the boundary conditions we consider, the space $\mathbf{V}$ is endowed with the scalar product

$$
((\boldsymbol{v}, \tilde{\boldsymbol{v}}))=(\nabla \boldsymbol{v}, \nabla \tilde{\boldsymbol{v}})+\int_{\mathcal{M}}\left(\frac{g p}{R \bar{T}}\right)^{2} \partial_{p} \boldsymbol{v} \cdot \partial_{p} \tilde{\boldsymbol{v}} \mathrm{d} \mathcal{M}+\int_{\Gamma_{i}}\left(\frac{g p_{1}}{R \bar{T}}\right)^{2} \boldsymbol{v} \cdot \tilde{\boldsymbol{v}} \mathrm{d} \Gamma_{i} .
$$

Again, we will not differentiate the notation of norms and scalar products between spaces of vector-valued functions and spaces of scalar functions. Therefore, $((\cdot, \cdot))$ and $\|\cdot\|$ will denote the scalar product and the norm in $\mathbf{V}$ as well. The spaces of interest for the triplet $(\boldsymbol{v}, T, q)$ are therefore

$$
H=\mathbf{H} \times L^{2} \times L^{2} \quad \text { and } \quad V=\mathbf{V} \times H^{1} \times H^{1} .
$$

Also, we shall make use of the space

$$
W=V \cap\left(H^{2}\right)^{4},
$$

when dealing with regularity of solutions. We then have the following Gelfand-Lions inclusions

$$
W \subset V \subset H \subset V^{\prime} \subset W^{\prime}
$$

with continuous injections and each space being dense in the next one.

3.4. Bilinear and trilinear forms. Having in mind the boundary conditions (3.14)(3.16), we observe the following: if $(\boldsymbol{v}, \theta, q),(\tilde{\boldsymbol{v}}, \tilde{\theta}, \tilde{q}) \in V$, then integration by parts yields

$$
\left\langle\mathcal{A}_{\boldsymbol{v}} \boldsymbol{v}, \tilde{\boldsymbol{v}}\right\rangle=\mu_{\boldsymbol{v}}(\nabla \boldsymbol{v}, \nabla \tilde{\boldsymbol{v}})+\nu_{\boldsymbol{v}} \int_{\mathcal{M}}\left(\frac{g p}{R \bar{T}}\right)^{2} \partial_{p} \boldsymbol{v} \cdot \partial_{p} \tilde{\boldsymbol{v}} \mathrm{d} \mathcal{M}+\alpha_{\boldsymbol{v}} \int_{\Gamma_{i}}\left(\frac{g p_{1}}{R \bar{T}}\right)^{2} \boldsymbol{v} \cdot \tilde{\boldsymbol{v}} \mathrm{d} \Gamma_{i},
$$

and

$$
\int_{\mathcal{M}} \nabla \Phi_{s}(x, y) \cdot \boldsymbol{v} \mathrm{d} \mathcal{M}=0
$$

Similarly,

$$
\begin{aligned}
\left\langle\mathcal{A}_{\theta} \theta, \tilde{\theta}\right\rangle= & \int_{\mathcal{M}} \mu_{T} \nabla \theta \cdot \nabla \tilde{\theta}+\nu_{T}\left(\frac{g p}{R \bar{T}}\right)^{2} \partial_{p} \theta \partial_{p} \tilde{\theta} \mathrm{d} \mathcal{M}+\alpha_{T} \int_{\Gamma_{i}}\left(\frac{g p_{1}}{R \bar{T}}\right)^{2} \theta \tilde{\theta} \mathrm{d} \Gamma_{i} \\
& -\nu_{T} \int_{\mathcal{M}}\left(\frac{g}{c_{p} \bar{T}}\right)^{2} \theta \tilde{\theta} \mathrm{d} \mathcal{M}+\frac{\nu_{T}}{c_{p}} \int_{\mathcal{M}} \frac{g^{2} p}{R \bar{T}^{2}}\left(\theta \partial_{p} \tilde{\theta}-\tilde{\theta} \partial_{p} \theta\right) \mathrm{d} \mathcal{M}
\end{aligned}
$$

and

$$
\left\langle\mathcal{A}_{q} q, \tilde{q}\right\rangle=\mu_{q}(\nabla q, \nabla \tilde{q})+\nu_{q} \int_{\mathcal{M}}\left(\frac{g p}{R \bar{T}}\right)^{2} \partial_{p} q \partial_{p} \tilde{q} \mathrm{~d} \mathcal{M}+\alpha_{q} \int_{\Gamma_{i}}\left(\frac{g p_{1}}{R \bar{T}}\right)^{2} q \tilde{q} \mathrm{~d} \Gamma_{i}
$$


For $(\boldsymbol{v}, \theta, q),(\tilde{\boldsymbol{v}}, \tilde{\theta}, \tilde{q}),\left(\boldsymbol{v}^{\#}, \theta^{\#}, q^{\#}\right) \in V$, we define the bilinear and trilinear forms as follows:

$$
\begin{aligned}
& a_{\boldsymbol{v}}(\boldsymbol{v}, \tilde{\boldsymbol{v}})=\mu_{\boldsymbol{v}}(\nabla \boldsymbol{v}, \nabla \tilde{\boldsymbol{v}})+\nu_{\boldsymbol{v}} \int_{\mathcal{M}}\left(\frac{g p}{R \bar{T}}\right)^{2} \partial_{p} \boldsymbol{v} \cdot \partial_{p} \tilde{\boldsymbol{v}} \mathrm{d} \mathcal{M}+\alpha_{\boldsymbol{v}} \int_{\Gamma_{i}}\left(\frac{g p_{1}}{R \bar{T}}\right)^{2} \boldsymbol{v} \cdot \tilde{\boldsymbol{v}} \mathrm{d} \Gamma_{i}, \\
& a_{T}(\theta, \tilde{\theta})=\mu_{T}(\nabla \theta, \nabla \tilde{\theta})+\nu_{T} \int_{\mathcal{M}}\left(\frac{g p}{R \bar{T}}\right)^{2} \partial_{p} \theta \partial_{p} \tilde{\theta} \mathrm{d} \mathcal{M}+\alpha_{T} \int_{\Gamma_{i}}\left(\frac{g p_{1}}{R \bar{T}}\right)^{2} \theta \tilde{\theta} \mathrm{d} \Gamma_{i}, \\
& a_{q}(q, \tilde{q})=\mu_{q}(\nabla q, \nabla \tilde{q})+\nu_{q} \int_{\mathcal{M}}\left(\frac{g p}{R \bar{T}}\right)^{2} \partial_{p} q \partial_{p} \tilde{q} \mathrm{~d} \mathcal{M}+\alpha_{q} \int_{\Gamma_{i}}\left(\frac{g p_{1}}{R \bar{T}}\right)^{2} q \tilde{q} \mathrm{~d} \Gamma_{i}, \\
& m_{\theta}(\theta, \tilde{\theta})=-\nu_{T} \int_{\mathcal{M}}\left(\frac{g}{c_{p} \bar{T}}\right)^{2} \theta \tilde{\theta} \mathrm{d} \mathcal{M}+\frac{\nu_{T}}{c_{p}} \int_{\mathcal{M}} \frac{g^{2} p}{R \bar{T}^{2}}\left(\theta \partial_{p} \tilde{\theta}-\tilde{\theta} \partial_{p} \theta\right) \mathrm{d} \mathcal{M}, \\
& b\left(\boldsymbol{v}, \tilde{\boldsymbol{v}}, \boldsymbol{v}^{\#}\right)=\int_{\mathcal{M}}\left((\boldsymbol{v} \cdot \nabla) \tilde{\boldsymbol{v}}+\omega \frac{\partial \tilde{\boldsymbol{v}}}{\partial p}\right) \cdot \boldsymbol{v}^{\#} \mathrm{~d} \mathcal{M}, \\
& e_{p}(\theta, \tilde{\boldsymbol{v}})=\int_{\mathcal{M}} \nabla \int_{p}^{p_{1}} \frac{R}{p^{\prime}}\left(\frac{p^{\prime}}{p_{0}}\right)^{R / c_{p}} \theta \mathrm{d} p^{\prime} \cdot \tilde{\boldsymbol{v}} \mathrm{d} \mathcal{M}, \\
& e_{c}(\boldsymbol{v}, \tilde{\boldsymbol{v}})=\int_{\mathcal{M}}(f \boldsymbol{k} \times \boldsymbol{v}) \cdot \tilde{\boldsymbol{v}} \mathrm{d} \mathcal{M} .
\end{aligned}
$$

Again, in order to keep the notation as simple as possible, the trilinear form $b$ could also have scalar functions in the last two arguments, meaning, for example, that

$$
b\left(\boldsymbol{v}, \tilde{\theta}, \theta^{\#}\right)=\int_{\mathcal{M}}\left(\boldsymbol{v} \cdot \nabla \tilde{\theta}+\omega \frac{\partial \tilde{\theta}}{\partial p}\right) \theta^{\#} \mathrm{~d} \mathcal{M} .
$$

It is clear that the $a_{i}$ 's $(i=\boldsymbol{v}, \theta, q)$ are bilinear continuous symmetric forms on $H^{1}$ that satisfy

$$
\mid a_{\boldsymbol{v}}(\boldsymbol{v}, \tilde{\boldsymbol{v}}) \leq C\|\boldsymbol{v}\|\|\tilde{\boldsymbol{v}}\|, \quad a_{T}(\theta, \tilde{\theta}) \leq C\|\theta\|\|\tilde{\theta}\|, \quad a_{q}(q, \tilde{q}) \leq C\|q\|\|\tilde{q}\|, \quad(C>0),
$$

for every $(\boldsymbol{v}, \theta, q),(\tilde{\boldsymbol{v}}, \tilde{\theta}, \tilde{q}) \in V$. Furthermore, they are coercive, for every $(\boldsymbol{v}, \theta, q) \in V$ :

$$
a_{\boldsymbol{v}}(\boldsymbol{v}, \boldsymbol{v}) \geq \kappa_{\boldsymbol{v}}\|\boldsymbol{v}\|^{2}, \quad a_{T}(\theta, \theta) \geq \kappa_{T}\|\theta\|^{2}, \quad a_{q}(q, q) \geq \kappa_{q}\|q\|^{2},
$$

where $\kappa_{\star}$ for $\star=\boldsymbol{v}, T, q$ is defined by

$$
\kappa_{\star}=\min \left(\mu_{\star}, \nu_{\star}, \alpha_{\star}\right)>0 .
$$

We also have that $m_{\theta}$ is bilinear continuous on $H^{1}$, with

$$
m_{\theta}(\theta, \tilde{\theta}) \leq C\|\theta\|\|\tilde{\theta}\|, \quad(C>0),
$$

and satisfies

$$
m_{\theta}(\theta, \theta) \geq-C|\theta|^{2}, \quad(C>0) .
$$

Finally, $e_{p}$ is bilinear continuous on either $H^{1} \times \mathbf{H}$ or $L^{2} \times \mathbf{V}$, and $e_{c}$ is bilinear continuous on $L^{2} \times L^{2}$. Hence, we have, for some $C>0$,

$$
\left|e_{p}(\theta, \boldsymbol{v})\right| \leq C\|\theta\|\left\|\boldsymbol{v}|, \quad| e_{p}(\theta, \boldsymbol{v})|\leq C| \theta|\|\boldsymbol{v}\|, \quad| e_{c}(\boldsymbol{v}, \tilde{\boldsymbol{v}})|\leq C| \boldsymbol{v}\right\| \tilde{\boldsymbol{v}} \mid .
$$

Also, we have the following result concerning the trilinear form $b$ (see [29, Lemmas 2.1 and 3.1]). 
Lemma 3.2. The trilinear form $b$ is continuous on $\mathbf{V} \times \mathbf{V} \times\left(\mathbf{V} \cap \mathbf{H}^{2}\right)$, and

$$
\left|b\left(\boldsymbol{v}, \tilde{\boldsymbol{v}}, \boldsymbol{v}^{\#}\right)\right| \leq C\|\boldsymbol{v}\||\tilde{\boldsymbol{v}}|^{1 / 2}\|\tilde{\boldsymbol{v}}\|^{1 / 2}\left\|\boldsymbol{v}^{\#}\right\|_{H^{2}}
$$

for some constant $C>0$. Furthermore,

$$
\left|b\left(\boldsymbol{v}, \tilde{\boldsymbol{v}}, \boldsymbol{v}^{\#}\right)\right| \leq C\|\boldsymbol{v}\|^{1 / 2}\|\boldsymbol{v}\|_{H^{2}}^{1 / 2}\|\tilde{\boldsymbol{v}}\|^{1 / 2}\|\tilde{\boldsymbol{v}}\|_{H^{2}}^{1 / 2}\left|\boldsymbol{v}^{\#}\right|,
$$

and

$$
\left|b\left(\boldsymbol{v}, \tilde{\boldsymbol{v}}, \boldsymbol{v}^{\#}\right)\right| \leq\left. C\|\boldsymbol{v}\|^{1 / 2}\|\boldsymbol{v}\|_{H^{2}}^{1 / 2}\|\tilde{\boldsymbol{v}}\| \boldsymbol{v}^{\#}\right|^{1 / 2}\left\|\boldsymbol{v}^{\#}\right\|^{1 / 2} .
$$

Also, using the incompressibility condition (2.3) and the boundary conditions $\boldsymbol{u} \cdot \boldsymbol{n}=0$ on $\partial \mathcal{M}$, we have

$$
b(\boldsymbol{v}, \tilde{\boldsymbol{v}}, \tilde{\boldsymbol{v}})=0 .
$$

Using Lemma 3.2, we then define the linear and bilinear continuous operators as follows:

$$
\begin{array}{lll}
A_{\boldsymbol{v}}: \mathbf{V} \rightarrow \mathbf{V}^{\prime}, & \left\langle A_{\boldsymbol{v}} \boldsymbol{v}, \tilde{\boldsymbol{v}}\right\rangle=a_{\boldsymbol{v}}(\boldsymbol{v}, \tilde{\boldsymbol{v}}), & \forall \boldsymbol{v}, \tilde{\boldsymbol{v}} \in \mathbf{V}, \\
A_{T}: H^{1} \rightarrow\left(H^{1}\right)^{\prime}, & \left\langle A_{T} \theta, \tilde{\theta}\right\rangle=a_{T}(\theta, \tilde{\theta}), & \forall \theta, \tilde{\theta} \in H^{1}, \\
A_{q}: H^{1} \rightarrow\left(H^{1}\right)^{\prime}, & \left\langle A_{q} q, \tilde{q}\right\rangle=a_{q}(q, \tilde{q}), & \forall q, \tilde{q} \in H^{1}, \\
M_{\theta}: H^{1} \rightarrow\left(H^{1}\right)^{\prime}, & \left\langle M_{\theta} \theta, \tilde{\theta}\right\rangle=m_{\theta}(\theta, \tilde{\theta}), & \forall \theta, \tilde{\theta} \in H^{1}, \\
E_{p}: L^{2} \rightarrow \mathbf{V}^{\prime}, & \left\langle E_{p} \theta, \tilde{\boldsymbol{v}}\right\rangle=e_{p}(\theta, \tilde{\boldsymbol{v}}), & \forall \theta \in L^{2}, \tilde{\boldsymbol{v}} \in \mathbf{V}, \\
E_{c}: \mathbf{H} \rightarrow \mathbf{H}, & \left\langle E_{c} \boldsymbol{v}, \tilde{\boldsymbol{v}}\right\rangle=e_{c}(\boldsymbol{v}, \tilde{\boldsymbol{v}}), & \forall \boldsymbol{v}, \tilde{\boldsymbol{v}} \in \mathbf{H} .
\end{array}
$$

Also, from the properties of the trilinear form $b$, we can define a bilinear operator

$$
B: \mathbf{V} \times \mathbf{V} \rightarrow\left(\mathbf{V} \cap\left(H^{2}\right)^{2}\right)^{\prime}
$$

acting as

$$
\left\langle B(\boldsymbol{v}, \tilde{\boldsymbol{v}}), \boldsymbol{v}^{\#}\right\rangle=b\left(\boldsymbol{v}, \tilde{\boldsymbol{v}}, \boldsymbol{v}^{\#}\right), \quad \forall \boldsymbol{v}, \tilde{\boldsymbol{v}} \in \mathbf{V}, \boldsymbol{v}^{\#} \in \mathbf{V} \cap\left(H^{2}\right)^{2}
$$

3.5. Quasi-strong solutions. Let $\boldsymbol{v}_{0} \in \mathbf{V}$ and $\theta_{0}, q_{0} \in L^{2}$. Assume $\left(\boldsymbol{S}_{\boldsymbol{v}}, S_{\theta}, S_{q}\right) \in$ $L^{2}\left(0, t_{1} ; H\right), q_{s} \in(0,1)$ and $t_{1}>0$. A vector $(\boldsymbol{v}, \theta, q)$ is a quasi-strong solution to (3.11)(3.19) if

$$
\begin{array}{ll}
\boldsymbol{v} \in C\left(\left[0, t_{1}\right] ; \mathbf{V}\right) \cap L^{2}\left(0, t_{1} ; H^{2}\right), & \theta, q \in C\left(\left[0, t_{1}\right] ; L^{2}\right) \cap L^{2}\left(0, t_{1} ; H^{1}\right), \\
\partial_{t} \boldsymbol{v} \in L^{2}\left(0, t_{1} ; \mathbf{H}\right), & \partial_{t} \theta, \partial_{t} q \in L^{2}\left(0, t_{1} ;\left(H^{1}\right)^{\prime}\right),
\end{array}
$$

and, for almost every $t \in\left[0, t_{1}\right]$ and every $(\tilde{\boldsymbol{v}}, \tilde{\theta}, \tilde{q}) \in \mathbf{H} \times H^{1} \times H^{1}$,

$$
\begin{aligned}
& \left\langle\partial_{t} \boldsymbol{v}, \tilde{\boldsymbol{v}}\right\rangle+a_{\boldsymbol{v}}(\boldsymbol{v}, \tilde{\boldsymbol{v}})+b(\boldsymbol{v}, \boldsymbol{v}, \tilde{\boldsymbol{v}})+e_{p}(\theta, \tilde{\boldsymbol{v}})+e_{c}(\boldsymbol{v}, \tilde{\boldsymbol{v}})=\left(\boldsymbol{S}_{\boldsymbol{v}}, \tilde{\boldsymbol{v}}\right) \\
& \left\langle\partial_{t} \theta, \tilde{\theta}\right\rangle+a_{\theta}(\theta, \tilde{\theta})+b(\boldsymbol{v}, \theta, \tilde{\theta})+m_{\theta}(\theta, \tilde{\theta})=\left(\frac{L}{c_{p}}\left(\frac{p_{0}}{p}\right)^{\frac{R}{c_{p}}} D\left(\omega, \theta, h_{q}\right), \tilde{\theta}\right)+\left(S_{\theta}, \tilde{\theta}\right), \\
& \left\langle\partial_{t} q, \tilde{q}\right\rangle+a_{q}(q, \tilde{q})+b(\boldsymbol{v}, q, \tilde{q})=-\left(D\left(\omega, \theta, h_{q}\right), \tilde{q}\right)+\left(S_{q}, \tilde{q}\right)
\end{aligned}
$$

for some $h_{q} \in L^{\infty}\left(\mathcal{M} \times\left(0, t_{1}\right)\right)$ which satisfies the variational inequality

$$
\left(\left[\tilde{q}-q_{s}\right]^{+}, 1\right)-\left(\left[q-q_{s}\right]^{+}, 1\right) \geq\left(h_{q}, \tilde{q}-q\right), \quad \text { a.e. } t \in\left[0, t_{1}\right], \quad \forall \tilde{q} \in H^{1} .
$$

Some remarks are in order. 
Remark 3.3. With a little abuse of notation, we wrote

$$
D\left(\omega, \theta, h_{q}\right)=\frac{1}{p} \omega^{-} h_{q} \widetilde{F}(p, \theta) .
$$

Compared to (3.10), we now have that $D\left(\omega, \theta, h_{q}\right)$ is a single-valued map, denoting by $h_{q}$ an (arbitrary) element of the set $H\left(q-q_{s}\right)$.

Remark 3.4. The variational inequality (3.32) expresses the fact that $h_{q}$ is an element of the sub-differential of the positive part function $q \mapsto\left(\left[q-q_{s}\right]^{+}, 1\right)$. Since

$$
\partial\left(\left[q-q_{s}\right]^{+}, 1\right)=H\left(q-q_{s}\right)
$$

it is easy to see that if $h_{q} \in H\left(q-q_{s}\right)$, then

$$
h_{q}(x, y, p)= \begin{cases}1, & \text { if } q>q_{s} \\ 0, & \text { if } q<q_{s}\end{cases}
$$

while if $q(x, y, p)=q_{s}$, we have

$$
h_{q}(x, y, p) \in[0,1]
$$

\section{An APPRoximated PROBlem}

In this section, we construct a family of regularized problems which approximate Problem (3.11)-(3.19) in a suitable sense. In this way, the limit of such approximated solutions will be shown to be a solution to our problem, in the sense made precise in Section 3.5. The proofs are based on a priori estimates and compactness arguments, and the variational inequality (3.32) plays an essential role.

4.1. Problem $\left(\mathbf{P}_{\varepsilon}\right)$. In order to introduce the approximated problems, we first define the real functions $H_{\varepsilon}$ and $K_{\varepsilon}$ approximating $H$ and $r^{+}$(the positive part of $r$ ). Namely, for $\varepsilon \in(0,1]$, let

$$
H_{\varepsilon}(r)=\left\{\begin{array}{ll}
0, & r \leq 0, \\
r / \varepsilon, & r \in(0, \varepsilon], \\
1, & r>\varepsilon,
\end{array} \quad K_{\varepsilon}(r)= \begin{cases}0, & r \leq 0, \\
r^{2} / 2 \varepsilon, & r \in(0, \varepsilon], \\
r-\varepsilon / 2, & r>\varepsilon .\end{cases}\right.
$$

It is straightforward to check that $K_{\varepsilon}^{\prime}=H_{\varepsilon}$,

$$
\left|H_{\varepsilon}\left(r_{1}\right)\right| \leq 1, \quad\left|H_{\varepsilon}\left(r_{1}\right)-H_{\varepsilon}\left(r_{2}\right)\right| \leq \frac{1}{\varepsilon}\left|r_{1}-r_{2}\right|, \quad \forall r_{1}, r_{2} \in \mathbb{R},
$$

and

$$
\left|K_{\varepsilon}\left(r_{1}\right)-K_{\varepsilon}\left(r_{2}\right)\right| \leq\left|r_{1}-r_{2}\right|, \quad \forall r_{1}, r_{2} \in \mathbb{R} .
$$

Moreover,

$$
\left|K_{\varepsilon}(r)-r\right| \leq \frac{\varepsilon}{2}, \quad \forall r \geq 0
$$


We then consider the following family of problems, depending on the parameter $\varepsilon$, and for which we seek local quasi-strong solutions for every fixed $\varepsilon>0$ :

$$
\begin{aligned}
& \left\langle\partial_{t} \boldsymbol{v}, \tilde{\boldsymbol{v}}\right\rangle+a_{\boldsymbol{v}}(\boldsymbol{v}, \tilde{\boldsymbol{v}})+b(\boldsymbol{v}, \boldsymbol{v}, \tilde{\boldsymbol{v}})+e_{p}(\theta, \tilde{\boldsymbol{v}})+e_{c}(\boldsymbol{v}, \tilde{\boldsymbol{v}})=\left(\boldsymbol{S}_{\boldsymbol{v}}, \tilde{\boldsymbol{v}}\right), \\
& \left\langle\partial_{t} \theta, \tilde{\theta}\right\rangle+a_{T}(\theta, \tilde{\theta})+b(\boldsymbol{v}, \theta, \tilde{\theta})+m_{\theta}(\theta, \tilde{\theta})=\left(\frac{L}{c_{p}}\left(\frac{p_{0}}{p}\right)^{\frac{R}{c_{p}}} D_{\varepsilon}(\omega, \theta, q), \tilde{\theta}\right)+\left(S_{\theta}, \tilde{\theta}\right), \\
& \left\langle\partial_{t} q, \tilde{q}\right\rangle+a_{q}(q, \tilde{q})+b(\boldsymbol{v}, q, \tilde{q})=-\left(D_{\varepsilon}(\omega, \theta, q), \tilde{q}\right)+\left(S_{q}, \tilde{q}\right) .
\end{aligned}
$$

Here,

$$
D_{\varepsilon}(\omega, \theta, q)=\frac{1}{p} \omega^{-} H_{\varepsilon}\left(q-q_{s}\right) \widetilde{F}(p, \theta),
$$

is now a well-defined map.

Now, we state the main result of this section, with the proof presented in the subsequent paragraphs.

Theorem 4.1. Assume that $\boldsymbol{v}_{0} \in \mathbf{V}$ and $\theta_{0}, q_{0} \in L^{2}$ are given and the forcing term $\left(\boldsymbol{S}_{\boldsymbol{v}}, S_{\theta}, S_{q}\right) \in L^{2}\left(0, t_{1} ; H\right)$. Let $q_{s} \in(0,1), t_{1}>0$ and $\varepsilon>0$ be fixed. Then there exists $t_{*}>0\left(t_{*} \leq t_{1}\right.$, independent of $\left.\varepsilon\right)$ and a quasi-strong solution $\left(\boldsymbol{v}^{\varepsilon}, \theta^{\varepsilon}, q^{\varepsilon}\right)$ to $\left(\mathbf{P}_{\varepsilon}\right)$ such that

$$
\boldsymbol{v}^{\varepsilon} \in C\left(\left[0, t_{*}\right) ; \mathbf{V}\right) \cap L^{2}\left(0, t_{*} ; H^{2}\right), \quad \partial_{t} \boldsymbol{v}^{\varepsilon} \in L^{2}\left(0, t_{*} ; \mathbf{H}\right),
$$

and

$$
\theta^{\varepsilon}, q^{\varepsilon} \in C\left(\left[0, t_{*}\right) ; L^{2}\right) \cap L^{2}\left(0, t_{*} ; H^{1}\right), \quad \partial_{t} \theta^{\varepsilon}, \partial_{t} q^{\varepsilon} \in L^{2}\left(0, t_{*} ;\left(H^{1}\right)^{\prime}\right) .
$$

Moreover, the following estimates hold true:

$$
\left\{\begin{array}{c}
\left\|\boldsymbol{v}^{\varepsilon}\right\|_{L^{\infty}\left(0, t_{*} ; \mathbf{V}\right)}+\left\|\boldsymbol{v}^{\varepsilon}\right\|_{L^{2}\left(0, t_{*} ; H^{2}\right)}+\left\|\left(\theta^{\varepsilon}, q^{\varepsilon}\right)\right\|_{L^{\infty}\left(0, t_{*} ; L^{2} \times L^{2}\right)} \\
+\left\|\left(\theta^{\varepsilon}, q^{\varepsilon}\right)\right\|_{L^{2}\left(0, t_{*} ; H^{1} \times H^{1}\right)} \leq \mathcal{Q}, \\
\left\|\partial_{t} \boldsymbol{v}^{\varepsilon}\right\|_{L^{2}\left(0, t_{*} ; \mathbf{H}\right)}+\left\|\left(\partial_{t} \theta^{\varepsilon}, \partial_{t} q^{\varepsilon}\right)\right\|_{L^{2}\left(0, t_{*} ;\left(H^{1}\right)^{\prime} \times\left(H^{1}\right)^{\prime}\right)} \leq \mathcal{Q},
\end{array}\right.
$$

where $\mathcal{Q}$ is a positive function independent of $\varepsilon$ defined by

$$
\mathcal{Q}:=\mathcal{Q}\left(t_{*},\left\|\boldsymbol{v}_{0}\right\|,\left|\theta_{0}\right|,\left|q_{0}\right|,\left\|\left(\boldsymbol{S}_{\boldsymbol{v}}, S_{\theta}, S_{q}\right)\right\|_{L^{2}\left(0, t_{1} ; H\right)}\right),
$$

which is increasing in all its arguments.

4.2. The Galerkin approximation. In order to establish an existence result for this problem, we implement the Galerkin method using the eigenvectors $e_{j}$ of $A=A_{\boldsymbol{v}} \oplus A_{T} \oplus A_{q}$ :

$$
A e_{j}=\lambda_{j} e_{j}, \quad j \geq 1, \quad 0<\lambda_{1} \leq \lambda_{2} \leq \cdots .
$$

The results of $[29$, Section 4.1] guarantee the following result.

Lemma 4.2. The eigenvectors $e_{j}$ of $A$ belong to $\left(H^{2}\right)^{4}$.

We denote by $A_{\boldsymbol{v}}^{1 / 2}$ the square root of $A_{\boldsymbol{v}}$; in particular,

$$
\left(A_{\boldsymbol{v}}^{1 / 2} \boldsymbol{v}, A_{\boldsymbol{v}}^{1 / 2} \tilde{\boldsymbol{v}}\right)=a_{\boldsymbol{v}}(\boldsymbol{v}, \tilde{\boldsymbol{v}}), \quad \forall \boldsymbol{v}, \tilde{\boldsymbol{v}} \in \mathbf{V} .
$$

Also denote by $A_{T}^{1 / 2}$ and $A_{q}^{1 / 2}$ the square roots of $A_{T}$ and $A_{q}$.

Note that the norm $\left|A_{\boldsymbol{v}}^{1 / 2} \boldsymbol{v}\right|$ is equivalent to the norm $\|\boldsymbol{v}\|$ for $\boldsymbol{v} \in \mathbf{V}$; also the norm $\left|A_{\boldsymbol{v}} \boldsymbol{v}\right|$ is equivalent to the norm $\|\boldsymbol{v}\|_{H^{2}}$ for $\boldsymbol{v} \in \mathbf{V} \cap\left(H^{2}\right)^{2}$. Similar results also hold for the operators $A_{\star}^{1 / 2}$ and $A_{\star}$ for $\star=T, q$ (cf. [29, Section 4]). 
4.3. A priori $L^{2}$-estimates (I). Here we prove a basic estimate on a Galerkin solution to $\left(\mathbf{P}_{\varepsilon}\right)$, which is contained in the following lemma.

Lemma 4.3. Fix $\varepsilon>0$ and let $\left(\boldsymbol{v}^{\varepsilon}, \theta^{\varepsilon}, q^{\varepsilon}\right)$ be a solution to $\left(\mathbf{P}_{\varepsilon}\right)$ with initial datum $\left(\boldsymbol{v}_{0}, \theta_{0}, q_{0}\right)$. Then

$$
\begin{aligned}
\sup _{t \in\left[0, t_{1}\right]}\left|\left(\boldsymbol{v}^{\varepsilon}, \theta^{\varepsilon}, q^{\varepsilon}\right)(t)\right|^{2}+\int_{0}^{t_{1}}\left\|\left(\boldsymbol{v}^{\varepsilon}, \theta^{\varepsilon}, q^{\varepsilon}\right)(t)\right\|^{2} \mathrm{~d} t \leq & C \mathrm{e}^{C t_{1}}\left(\left|\left(\boldsymbol{v}_{0}, \theta_{0}, q_{0}\right)\right|^{2}\right. \\
& \left.+\left\|\left(\boldsymbol{S}_{\boldsymbol{v}}, S_{T}, S_{q}\right)\right\|_{L^{2}\left(0, t_{1} ; H\right)}^{2}\right) .
\end{aligned}
$$

Proof. In what follows, $C$ is an absolute constant independent of $\varepsilon$ and also independent of the initial data $\left(\boldsymbol{v}_{0}, \theta_{0}, q_{0}\right)$ and the forcing terms $\left(\boldsymbol{S}_{\boldsymbol{v}}, S_{\theta}, S_{q}\right)$. We start from the estimate on the velocity. Taking the $L^{2}$-scalar of the $\boldsymbol{v}$-equation in $\left(\mathbf{P}_{\varepsilon}\right)$ with $\boldsymbol{v}^{\varepsilon}$, we obtain the energy equation

$$
\frac{1}{2} \frac{\mathrm{d}}{\mathrm{d} t}\left|\boldsymbol{v}^{\varepsilon}\right|^{2}+a_{\boldsymbol{v}}\left(\boldsymbol{v}^{\varepsilon}, \boldsymbol{v}^{\varepsilon}\right)=-e_{p}\left(\theta^{\varepsilon}, \boldsymbol{v}^{\varepsilon}\right)-e_{c}\left(\boldsymbol{v}^{\varepsilon}, \boldsymbol{v}^{\varepsilon}\right)+\left(\boldsymbol{S}_{\boldsymbol{v}}, \boldsymbol{v}^{\varepsilon}\right)
$$

Each term of the right hand side above can be respectively estimated as

$$
\begin{aligned}
& \left|e_{p}\left(\theta^{\varepsilon}, \boldsymbol{v}^{\varepsilon}\right)\right| \leq C\left\|\theta^{\varepsilon}\right\|\left|\boldsymbol{v}^{\varepsilon}\right| \leq \frac{\kappa_{T}}{2}\left\|\theta^{\varepsilon}\right\|^{2}+C\left|\boldsymbol{v}^{\varepsilon}\right|^{2}, \\
& e_{c}\left(\boldsymbol{v}^{\varepsilon}, \boldsymbol{v}^{\varepsilon}\right)=0, \\
& \left|\left(\boldsymbol{S}_{\boldsymbol{v}}, \boldsymbol{v}^{\varepsilon}\right)\right| \leq\left|\boldsymbol{S}_{\boldsymbol{v}} \| \boldsymbol{v}^{\varepsilon}\right| \leq C\left|\boldsymbol{S}_{\boldsymbol{v}}\right|^{2}+\frac{\kappa_{\boldsymbol{v}}}{2}\left|\boldsymbol{v}^{\varepsilon}\right|^{2} .
\end{aligned}
$$

Therefore, we find

$$
\frac{\mathrm{d}}{\mathrm{d} t}\left|\boldsymbol{v}^{\varepsilon}\right|^{2}+\kappa_{\boldsymbol{v}}\left\|\boldsymbol{v}^{\varepsilon}\right\|^{2} \leq C\left|\boldsymbol{v}^{\varepsilon}\right|^{2}+\kappa_{T}\left\|\theta^{\varepsilon}\right\|^{2}+C\left|\boldsymbol{S}_{\boldsymbol{v}}\right|^{2}
$$

Turning to the $\theta$-equation and applying the same reasoning, we find that

$$
\frac{1}{2} \frac{\mathrm{d}}{\mathrm{d} t}\left|\theta^{\varepsilon}\right|^{2}+a_{T}\left(\theta^{\varepsilon}, \theta^{\varepsilon}\right)+m_{\theta}\left(\theta^{\varepsilon}, \theta^{\varepsilon}\right)=\left(\frac{L}{c_{p}}\left(\frac{p_{0}}{p}\right)^{\frac{R}{c_{p}}} D_{\varepsilon}\left(\omega^{\varepsilon}, \theta^{\varepsilon}, q^{\varepsilon}\right), \theta^{\varepsilon}\right)+\left(S_{\theta}, \theta^{\varepsilon}\right),
$$

As noted in (3.23), we have $m_{\theta}\left(\theta^{\varepsilon}, \theta^{\varepsilon}\right) \geq-C\left|\theta^{\varepsilon}\right|^{2}$. Moreover,

$$
\begin{aligned}
\left(\frac{L}{c_{p}}\left(\frac{p_{0}}{p}\right)^{\frac{R}{c_{p}}} D_{\varepsilon}\left(\omega^{\varepsilon}, \theta^{\varepsilon}, q^{\varepsilon}\right), \theta^{\varepsilon}\right) & \leq\left|D_{\varepsilon}\left(\omega^{\varepsilon}, \theta^{\varepsilon}, q^{\varepsilon}\right)\left\|\theta^{\varepsilon}|\leq C| \omega^{\varepsilon}\right\| \theta^{\varepsilon}\right| \\
& \leq C\left\|\boldsymbol{v}^{\varepsilon}\right\|\left|\theta^{\varepsilon}\right| \leq \frac{\kappa_{\boldsymbol{v}}}{8}\left\|\boldsymbol{v}^{\varepsilon}\right\|^{2}+C\left|\theta^{\varepsilon}\right|^{2} .
\end{aligned}
$$

From the trivial estimate

$$
\left|\left(S_{\theta}, \theta^{\varepsilon}\right)\right| \leq C\left|S_{\theta}\right|^{2}+\frac{\kappa_{T}}{2}\left|\theta^{\varepsilon}\right|^{2},
$$

we learn that

$$
\frac{\mathrm{d}}{\mathrm{d} t}\left|\theta^{\varepsilon}\right|^{2}+\kappa_{T}\left\|\theta^{\varepsilon}\right\|^{2} \leq \frac{\kappa_{\boldsymbol{v}}}{4}\left\|\boldsymbol{v}^{\varepsilon}\right\|^{2}+C\left|\theta^{\varepsilon}\right|^{2}+C\left|S_{\theta}\right|^{2} .
$$

Finally, a similar estimate can be deduced for $q^{\varepsilon}$. Indeed, from $\left(\mathbf{P}_{\varepsilon}\right)$ we find

As before,

$$
\frac{1}{2} \frac{\mathrm{d}}{\mathrm{d} t}\left|q^{\varepsilon}\right|^{2}+a_{q}\left(q^{\varepsilon}, q^{\varepsilon}\right)=-\left(D_{\varepsilon}\left(\omega^{\varepsilon}, \theta^{\varepsilon}, q^{\varepsilon}\right), q^{\varepsilon}\right)+\left(S_{q}, q^{\varepsilon}\right) .
$$

$$
\left|\left(D_{\varepsilon}\left(\omega^{\varepsilon}, \theta^{\varepsilon}, q^{\varepsilon}\right), q^{\varepsilon}\right)\right| \leq \frac{\kappa_{\boldsymbol{v}}}{8}\left\|\boldsymbol{v}^{\varepsilon}\right\|^{2}+C\left|q^{\varepsilon}\right|^{2}
$$


and

so that

$$
\left|\left(S_{q}, q^{\varepsilon}\right)\right| \leq C\left|S_{q}\right|^{2}+\frac{\kappa_{q}}{2}\left|q^{\varepsilon}\right|^{2},
$$

Adding together (4.9), (4.10), and (4.11) we obtain

$$
\begin{aligned}
\frac{\mathrm{d}}{\mathrm{d} t}\left[\left|\boldsymbol{v}^{\varepsilon}\right|^{2}+\left|\theta^{\varepsilon}\right|^{2}+\left|q^{\varepsilon}\right|^{2}\right] & +\kappa\left(\left\|\boldsymbol{v}^{\varepsilon}\right\|^{2}+\left\|\theta^{\varepsilon}\right\|^{2}+\left\|q^{\varepsilon}\right\|^{2}\right) \\
& \leq C\left(\left|\boldsymbol{v}^{\varepsilon}\right|^{2}+\left|\theta^{\varepsilon}\right|^{2}+\left|q^{\varepsilon}\right|^{2}\right)+C\left(\left|\boldsymbol{S}_{\boldsymbol{v}}\right|^{2}+\left|S_{\theta}\right|^{2}+\left|S_{q}\right|^{2}\right),
\end{aligned}
$$

with $\kappa=\min \left\{\kappa_{\boldsymbol{v}} / 2, \kappa_{T}, \kappa_{q}\right\}>0$. The conclusion (4.7) follows from a standard application of the Gronwall lemma.

4.4. Change of equations for $\boldsymbol{v}$. In order to prove the $H^{1}$-regularity on the velocity, we first study the linear problem for the velocity in $\left(\mathbf{P}_{\varepsilon}\right)_{1}$ and show that the solution of the linear problem enjoys the $H^{1}$-regularity; then we prove the $H^{1}$ regularity of the solution for the nonlinear problem of $\left(\mathbf{P}_{\varepsilon}\right)_{1}$ in a short time. We state the problems in this subsection and establish the desired a priori estimates in the next subsection.

We write the equation $\left(\mathbf{P}_{\varepsilon}\right)_{1}$ in the functional form:

$$
\left\{\begin{array}{l}
\frac{\mathrm{d} \boldsymbol{v}}{\mathrm{d} t}+A_{\boldsymbol{v}} \boldsymbol{v}+B_{\boldsymbol{v}}(\boldsymbol{v}, \boldsymbol{v})+E_{c} \boldsymbol{v}=\boldsymbol{S}_{\boldsymbol{v}}-E_{p} \theta, \\
\boldsymbol{v}(0)=\boldsymbol{v}_{0}
\end{array}\right.
$$

where the first equation is understood in $\left(\mathbf{V} \cap\left(H^{2}\right)^{2}\right)^{\prime}$, the dual space of the $H^{2}$-like space for the velocity field, and $\theta$ is given in the space $L^{2}\left(0, t_{1} ; H^{1}\right)$ and hence $E_{p} \theta$ belongs to $L^{2}\left(0, t_{1} ; L^{2}\right)$. The linear equation that we consider reads

$$
\left\{\begin{array}{l}
\frac{\mathrm{d} \boldsymbol{v}^{*}}{\mathrm{~d} t}+A_{\boldsymbol{v}} \boldsymbol{v}^{*}+E_{c} \boldsymbol{v}^{*}=\boldsymbol{S}_{\boldsymbol{v}}-E_{p} \theta \\
\boldsymbol{v}^{*}(0)=\boldsymbol{v}_{0}
\end{array}\right.
$$

We then set $\boldsymbol{v}^{\prime}=\boldsymbol{v}-\boldsymbol{v}^{*}$, and by subtracting (4.14) from (4.13), we see that $\boldsymbol{v}^{\prime}$ satisfies

$$
\left\{\begin{array}{l}
\frac{\mathrm{d} \boldsymbol{v}^{\prime}}{\mathrm{d} t}+A_{\boldsymbol{v}} \boldsymbol{v}^{\prime}+B_{\boldsymbol{v}}\left(\boldsymbol{v}^{\prime}, \boldsymbol{v}^{\prime}\right)+B_{\boldsymbol{v}}\left(\boldsymbol{v}^{\prime}, \boldsymbol{v}^{*}\right)+B_{\boldsymbol{v}}\left(\boldsymbol{v}^{*}, \boldsymbol{v}^{\prime}\right)+E_{c} \boldsymbol{v}^{\prime}=-B_{\boldsymbol{v}}\left(\boldsymbol{v}^{*}, \boldsymbol{v}^{*}\right), \\
\boldsymbol{v}^{\prime}(0)=0
\end{array}\right.
$$

Our goal in the following subsection is to prove the a priori estimates for $\boldsymbol{v}^{*}$ in (4.14) and $\boldsymbol{v}^{\prime}$ in (4.15), and the existence and uniqueness of solutions $\boldsymbol{v}$ for (4.13).

4.5. A priori $H^{1}$-estimates for the velocity (II). In this subsection, we are aiming to derive the $L^{\infty}\left(H^{1}\right)$ a priori estimates for the equations (4.13)-(4.15). We start with the a priori estimate for $\boldsymbol{v}^{*}$ in (4.14).

Lemma 4.4. Assume that $\boldsymbol{v}_{0} \in \mathbf{V}, \theta \in L^{2}\left(0, t_{1} ; H^{1}\right)$, and $\boldsymbol{S}_{\boldsymbol{v}} \in L^{2}\left(0, t_{1} ; L^{2}\right)$, and let $\boldsymbol{v}^{*}$ be the solution to (4.14). Then $\boldsymbol{v}^{*}$ belongs to $L^{\infty}\left(0, t_{1} ; \mathbf{V}\right) \cap L^{2}\left(0, t_{1} ; H^{2}\right)$ and satisfies

$$
\sup _{t \in\left[0, t_{1}\right]}\left|A_{\boldsymbol{v}}^{1 / 2} \boldsymbol{v}^{*}(t)\right|^{2}+\int_{0}^{t_{1}}\left|A_{\boldsymbol{v}} \boldsymbol{v}^{*}(t)\right|^{2} \mathrm{~d} t \leq C \mathrm{e}^{C t_{1}}\left(\left\|\boldsymbol{v}_{0}\right\|^{2}+\int_{0}^{t_{1}}\left(\left|\boldsymbol{S}_{\boldsymbol{v}}(t)\right|^{2}+\|\theta(t)\|^{2}\right) \mathrm{d} t\right) .
$$


Proof. Taking the $L^{2}$-scalar of $(4.14)_{1}$ with $\boldsymbol{v}^{*}$ and using the estimates in (4.8), we obtain

$$
\frac{\mathrm{d}}{\mathrm{d} t}\left|\boldsymbol{v}^{*}\right|^{2}+\kappa_{\boldsymbol{v}}\left\|\boldsymbol{v}^{*}\right\|^{2} \leq C\left(\left|\boldsymbol{v}^{*}\right|^{2}+\|\theta\|^{2}\right)+\left|\boldsymbol{S}_{\boldsymbol{v}}\right|^{2}
$$

which, by the Gronwall lemma, implies that

$$
\sup _{t \in\left[0, t_{1}\right]}\left|\boldsymbol{v}^{*}(t)\right|^{2}+\kappa_{\boldsymbol{v}} \int_{0}^{t_{1}}\left\|\boldsymbol{v}^{*}(t)\right\|^{2} \mathrm{~d} t \leq C \mathrm{e}^{C t_{1}}\left(\left|\boldsymbol{v}_{0}\right|^{2}+\int_{0}^{t_{1}}\left(\left|\boldsymbol{S}_{\boldsymbol{v}}(t)\right|^{2}+\|\theta(t)\|^{2}\right) \mathrm{d} t\right) .
$$

We now multiply $(4.14)_{1}$ by $A_{\boldsymbol{v}} \boldsymbol{v}^{*}$ in $L^{2}$, and we find

$$
\begin{aligned}
\frac{1}{2} \frac{\mathrm{d}}{\mathrm{d} t}\left|A_{\boldsymbol{v}}^{1 / 2} \boldsymbol{v}^{*}\right|^{2}+\left|A_{\boldsymbol{v}} \boldsymbol{v}^{*}\right|^{2} & =-e_{p}\left(\theta, A_{\boldsymbol{v}} \boldsymbol{v}^{*}\right)-e_{c}\left(\boldsymbol{v}^{*}, A_{\boldsymbol{v}} \boldsymbol{v}^{*}\right)-\left(\boldsymbol{S}_{\boldsymbol{v}}, A_{\boldsymbol{v}} \boldsymbol{v}^{*}\right) \\
& \leq C\|\theta\|\left|A_{\boldsymbol{v}} \boldsymbol{v}^{*}\right|+C\left|\boldsymbol{v}^{*}\right|\left|A_{\boldsymbol{v}} \boldsymbol{v}^{*}\right|+\left|\boldsymbol{S}_{\boldsymbol{v}} \| A_{\boldsymbol{v}} \boldsymbol{v}^{*}\right|
\end{aligned}
$$

Using Young's inequality for the right-hand side of (4.18), we arrive at

$$
\frac{\mathrm{d}}{\mathrm{d} t}\left|A_{\boldsymbol{v}}^{1 / 2} \boldsymbol{v}^{*}\right|^{2}+\left|A_{\boldsymbol{v}} \boldsymbol{v}^{*}\right|^{2} \leq C\left(\|\theta\|^{2}+\left|\boldsymbol{v}^{*}\right|^{2}+\left|\boldsymbol{S}_{\boldsymbol{v}}\right|^{2}\right),
$$

which, by the Gronwall lemma again, shows that

$$
\begin{aligned}
\sup _{t \in\left[0, t_{1}\right]}\left|A_{\boldsymbol{v}}^{1 / 2} \boldsymbol{v}^{*}(t)\right|^{2}+\int_{0}^{t_{1}}\left|A_{\boldsymbol{v}} \boldsymbol{v}^{*}(t)\right|^{2} \mathrm{~d} t \leq & C \mathrm{e}^{C t_{1}}\left\|\boldsymbol{v}_{0}\right\|^{2} \\
& +C \mathrm{e}^{C t_{1}}\left(\int_{0}^{t_{1}}\left(\left|\boldsymbol{S}_{\boldsymbol{v}}(t)\right|^{2}+\left|\boldsymbol{v}^{*}(t)\right|^{2}+\|\theta(t)\|^{2}\right) \mathrm{d} t\right) .
\end{aligned}
$$

The conclusion (4.16) then follows from (4.17).

We now turn to the a priori estimate for $\boldsymbol{v}^{\prime}$ in (4.15).

Lemma 4.5. Assume that $\boldsymbol{v}^{*}$ belongs to $L^{\infty}\left(0, t_{1} ; \mathbf{V}\right) \cap L^{2}\left(0, t_{1} ; H^{2}\right)$, and $\boldsymbol{v}^{\prime}$ is a solution to (4.15). Then there exists $t_{*}>0\left(t_{*} \leq t_{1}\right)$ such that $\boldsymbol{v}^{\prime}$ belongs to $L^{\infty}\left(0, t_{*} ; \mathbf{V}\right) \cap L^{2}\left(0, t_{*} ; H^{2}\right)$ and satisfies

$$
\sup _{t \in\left[0, t_{*}\right)}\left|A_{\boldsymbol{v}}^{1 / 2} \boldsymbol{v}^{\prime}(t)\right|^{2}+\int_{0}^{t_{*}}\left|A_{\boldsymbol{v}} \boldsymbol{v}^{\prime}(t)\right|^{2} \mathrm{~d} t \leq \mathcal{Q}_{1}\left(\left\|\boldsymbol{v}^{*}\right\|_{L^{\infty}\left(0, t_{1} ; \mathbf{V}\right)},\left\|\boldsymbol{v}^{*}\right\|_{L^{2}\left(0, t_{1} ; H^{2}\right)}\right),
$$

where $\mathcal{Q}_{1}$ is a positive function.

Proof. Multiplying $(4.15)_{1}$ by $A_{\boldsymbol{v}} \boldsymbol{v}^{\prime}$ in $L^{2}$, we obtain that

$$
\begin{aligned}
\frac{1}{2} \frac{\mathrm{d}}{\mathrm{d} t}\left|A_{\boldsymbol{v}}^{1 / 2} \boldsymbol{v}^{\prime}\right|^{2}+\left|A_{\boldsymbol{v}} \boldsymbol{v}^{\prime}\right|^{2}= & -e_{c}\left(\boldsymbol{v}^{\prime}, A_{\boldsymbol{v}} \boldsymbol{v}^{\prime}\right)-b\left(\boldsymbol{v}^{\prime}, \boldsymbol{v}^{*}, A_{\boldsymbol{v}} \boldsymbol{v}^{\prime}\right)-b\left(\boldsymbol{v}^{*}, \boldsymbol{v}^{\prime}, A_{\boldsymbol{v}} \boldsymbol{v}^{\prime}\right) \\
& -b\left(\boldsymbol{v}^{*}, \boldsymbol{v}^{*}, A_{\boldsymbol{v}} \boldsymbol{v}^{\prime}\right)-b\left(\boldsymbol{v}^{\prime}, \boldsymbol{v}^{\prime}, A_{\boldsymbol{v}} \boldsymbol{v}^{\prime}\right) .
\end{aligned}
$$

Using Young's inequality and (3.26) in Lemma 3.2 for the $b$-terms, we bound each term in the right-hand side of (4.21) as follows: using the fact that the norm $\left|A_{\boldsymbol{v}} \boldsymbol{v}^{\prime}\right|$ is equivalent 
to the norm $\left\|\boldsymbol{v}^{\prime}\right\|_{H^{2}}$, and that the norm $\left|A_{\boldsymbol{v}}^{1 / 2} \boldsymbol{v}^{\prime}\right|$ is equivalent to the norm $\left\|\boldsymbol{v}^{\prime}\right\|$, we obtain

$$
\begin{aligned}
& \left|e_{c}\left(\boldsymbol{v}^{\prime}, A_{\boldsymbol{v}} \boldsymbol{v}^{\prime}\right)\right| \leq \frac{1}{12}\left|A_{\boldsymbol{v}} \boldsymbol{v}^{\prime}\right|^{2}+C\left|\boldsymbol{v}^{\prime}\right|^{2} \\
& \left|b\left(\boldsymbol{v}^{\prime}, \boldsymbol{v}^{*}, A_{\boldsymbol{v}} \boldsymbol{v}^{\prime}\right)\right| \leq\left. C\left\|\boldsymbol{v}^{\prime}\right\|\right|^{1 / 2}\left\|\boldsymbol{v}^{*}\right\|^{1 / 2}\left\|\boldsymbol{v}^{*}\right\|_{H^{2}}^{1 / 2}\left|A_{\boldsymbol{v}} \boldsymbol{v}^{\prime}\right|^{3 / 2} \leq \frac{1}{12}\left|A_{\boldsymbol{v}} \boldsymbol{v}^{\prime}\right|^{2}+C\left\|\boldsymbol{v}^{\prime}\right\|^{2}\left\|\boldsymbol{v}^{*}\right\|^{2}\left\|\boldsymbol{v}^{*}\right\|_{H^{2}}^{2}, \\
& \left|b\left(\boldsymbol{v}^{*}, \boldsymbol{v}^{\prime}, A_{\boldsymbol{v}} \boldsymbol{v}^{\prime}\right)\right| \leq \frac{1}{12}\left|A_{\boldsymbol{v}} \boldsymbol{v}^{\prime}\right|^{2}+C\left\|\boldsymbol{v}^{\prime}\right\|^{2}\left\|\boldsymbol{v}^{*}\right\|^{2}\left\|\boldsymbol{v}^{*}\right\|_{H^{2}}^{2}, \\
& \left|b\left(\boldsymbol{v}^{*}, \boldsymbol{v}^{*}, A_{\boldsymbol{v}} \boldsymbol{v}^{\prime}\right)\right| \leq C\left\|\boldsymbol{v}^{*}\right\|\left\|\boldsymbol{v}^{*}\right\|_{H^{2}}\left|A_{\boldsymbol{v}} \boldsymbol{v}^{\prime}\right| \leq \frac{1}{12}\left|A_{\boldsymbol{v}} \boldsymbol{v}^{\prime}\right|^{2}+C\left\|\boldsymbol{v}^{*}\right\|^{2}\left\|\boldsymbol{v}^{*}\right\|_{H^{2}}^{2}, \\
& \left|b\left(\boldsymbol{v}^{\prime}, \boldsymbol{v}^{\prime}, A_{\boldsymbol{v}} \boldsymbol{v}^{\prime}\right)\right| \leq c_{\star}\left\|\boldsymbol{v}^{\prime}\right\|\left|A_{\boldsymbol{v}} \boldsymbol{v}^{\prime}\right|^{2}
\end{aligned}
$$

Taking all these bounds into account, we infer from (4.21) that

$$
\frac{\mathrm{d}}{\mathrm{d} t}\left|A_{\boldsymbol{v}}^{1 / 2} \boldsymbol{v}^{\prime}\right|^{2}+\left(1-c_{\star}\left|A_{\boldsymbol{v}}^{1 / 2} \boldsymbol{v}^{\prime}\right|\right)\left|A_{\boldsymbol{v}} \boldsymbol{v}^{\prime}\right|^{2} \leq \eta_{1}(t)\left|A_{\boldsymbol{v}}^{1 / 2} \boldsymbol{v}^{\prime}\right|^{2}+\eta_{2}(t),
$$

with

$$
\eta_{1}(t)=c+\eta_{2}(t), \quad \eta_{2}(t)=c\left\|\boldsymbol{v}^{*}\right\|^{2}\left\|\boldsymbol{v}^{*}\right\|_{H^{2}}^{2} .
$$

As long as $1-c_{\star}\left|A_{\boldsymbol{v}}^{1 / 2} \boldsymbol{v}^{\prime}\right| \geq 1 / 2$, that is

$$
\left|A_{\boldsymbol{v}}^{1 / 2} \boldsymbol{v}^{\prime}\right| \leq \frac{1}{2 c_{\star}}
$$

we then have, by Gronwall lemma and since $\boldsymbol{v}^{\prime}(0)=0$, on some interval of time $\left(0, t_{*}\right)$ :

$$
\left|A_{\boldsymbol{v}}^{1 / 2} \boldsymbol{v}^{\prime}\right|^{2} \leq \int_{0}^{t} \eta_{2}(t) \mathrm{e}^{\int_{\tau}^{t} \eta_{1}(s) \mathrm{d} s} \mathrm{~d} \tau, \quad \forall t \in\left(0, t_{*}\right) .
$$

In fact, $t_{*}>0$ can be chosen as the minimum between $t_{1}$ and $t_{2}$, where $t_{2}$ is either $+\infty$ or the first time at which

$$
\int_{0}^{t_{2}} \eta_{2}(s) \mathrm{d} s=\frac{1}{4 c_{\star}^{2}} \mathrm{e}^{-\int_{0}^{t_{1}} \eta_{1}(s) \mathrm{d} s} .
$$

In this way, we will then have

$$
\left|A_{\boldsymbol{v}}^{1 / 2} \boldsymbol{v}^{\prime}(t)\right|^{2} \leq \frac{1}{4 c_{\star}^{2}}, \quad \forall t \in\left(0, t_{*}\right),
$$

and returning to (4.23) we find the estimate (4.20).

We now study the nonlinear equations (4.13) for $\boldsymbol{v}$ and we have the following.

Lemma 4.6. Assume that $\boldsymbol{v}_{0} \in \mathbf{V}, \theta \in L^{2}\left(0, t_{1} ; H^{1}\right)$ and $\boldsymbol{S}_{\boldsymbol{v}} \in L^{2}\left(0, t_{1} ; \mathbf{H}\right)$. Then there exist $t_{*}>0\left(t_{*} \leq t_{1}\right)$ and a unique solution $\boldsymbol{v}$ to $(4.13)$ which belongs to $L^{\infty}\left(0, t_{*} ; \mathbf{V}\right) \cap$ $L^{2}\left(0, t_{*} ; H^{2}\right)$ and satisfies

$$
\sup _{t \in\left[0, t_{*}\right)}\left|A_{\boldsymbol{v}}^{1 / 2} \boldsymbol{v}(t)\right|^{2}+\int_{0}^{t_{*}}\left|A_{\boldsymbol{v}} \boldsymbol{v}(t)\right|^{2} \mathrm{~d} t \leq \mathcal{Q}_{2}\left(t_{*},\left\|\boldsymbol{v}_{0}\right\|,\|\theta\|_{L^{2}\left(0, t_{1} ; H^{1}\right)},\left\|\boldsymbol{S}_{\boldsymbol{v}}\right\|_{L^{2}\left(0, t_{1} ; L^{2}\right)}\right),
$$

where $\mathcal{Q}_{2}$ is a positive function, increasing in all arguments. 
Proof. The existence of $\boldsymbol{v}$ of (4.13) follows from the existence of $\boldsymbol{v}^{*}$, solution to (4.14), and $\boldsymbol{v}^{\prime}$ solution to (4.15), based on the standard Galerkin approximation procedure. Moreover estimate (4.24) follows from the estimates (4.16) and (4.20). We are left to prove the uniqueness.

Consider two solutions $\boldsymbol{v}_{1}, \boldsymbol{v}_{2}$ of (4.13) belonging to $L^{\infty}\left(0, t_{*} ; \mathbf{V}\right) \cap L^{2}\left(0, t_{*} ; H^{2}\right)$, and let $\tilde{\boldsymbol{v}}=\boldsymbol{v}_{1}-\boldsymbol{v}_{2}$. Then $\tilde{\boldsymbol{v}}$ satisfies

$$
\left\{\begin{array}{l}
\frac{\mathrm{d} \tilde{\boldsymbol{v}}}{\mathrm{d} t}+A_{\boldsymbol{v}} \tilde{\boldsymbol{v}}+B_{\boldsymbol{v}}\left(\tilde{\boldsymbol{v}}, \boldsymbol{v}_{1}\right)+B\left(\boldsymbol{v}_{2}, \tilde{\boldsymbol{v}}\right)+E_{c}(\tilde{\boldsymbol{v}})=0 \\
\tilde{\boldsymbol{v}}(0)=0
\end{array}\right.
$$

Taking the $L^{2}$-scalar product between $(4.25)_{1}$ and $A_{\boldsymbol{v}} \tilde{\boldsymbol{v}}$ and using similar estimates in (4.22) for the $b, e_{c}$ terms, we obtain

$$
\frac{\mathrm{d}}{\mathrm{d} t}\left|A_{\boldsymbol{v}}^{1 / 2} \tilde{\boldsymbol{v}}\right|^{2}+\left|A_{\boldsymbol{v}} \tilde{\boldsymbol{v}}\right|^{2} \leq \eta(t)\left|A_{\boldsymbol{v}}^{1 / 2} \tilde{\boldsymbol{v}}\right|^{2}
$$

where

$$
\eta(t)=c\left(1+\left\|\boldsymbol{v}_{1}\right\|^{2}\left\|\boldsymbol{v}_{1}\right\|_{H^{2}}^{2}+\left\|\boldsymbol{v}_{2}\right\|^{2}\left\|\boldsymbol{v}_{2}\right\|_{H^{2}}^{2}\right) \in L^{1}\left(0, t_{*}\right)
$$

for some $c>0$. The uniqueness follows by the Gronwall lemma.

Remark 4.7. We remark that the equations (4.13)-(4.15) are independent of $\varepsilon$, and thus the estimates (4.16), (4.20), and (4.24) are independent of $\varepsilon$ and so is the choice of $t_{*}$ in Lemmas 4.5-4.6.

4.6. Local well-posedness of $\left(\mathbf{P}_{\varepsilon}\right)$. With the a priori estimates in Lemmas 4.3-4.6 at hand, we are now ready to prove Theorem 4.1.

Proof of Theorem 4.1. Instead of solving $\left(\mathbf{P}_{\varepsilon}\right)$, we solve $\left(\mathbf{P}_{\varepsilon}\right)_{2,3}$ coupled (through $\theta$ ) with (4.14)-(4.15). We apply the standard Galerkin approximation procedure for the unknowns $\left(\boldsymbol{v}^{*}, \boldsymbol{v}^{\prime}, \theta, q\right)$ using the eigenvectors of $A$ introduced in (4.6). The a prori estimates (4.7), (4.16), and (4.20) show that we have uniform bounds independent of $\varepsilon$. It is then straightforward to pass to the limit, and we obtain the existence of $\left(\boldsymbol{v}^{\varepsilon *}, \boldsymbol{v}^{\varepsilon \prime}, \theta^{\varepsilon}, q^{\varepsilon}\right)$ for (4.14)(4.15), $\left(\mathbf{P}_{\varepsilon}\right)_{2,3}$ and hence the existence of $\left(\boldsymbol{v}^{\varepsilon}, \theta^{\varepsilon}, q^{\varepsilon}\right)$ for $\left(\mathbf{P}_{\varepsilon}\right)$ by letting $\boldsymbol{v}^{\varepsilon}=\boldsymbol{v}^{\varepsilon *}+\boldsymbol{v}^{\varepsilon}$.

The first estimate $(4.5)_{1}$ then follows from the a priori estimates (4.7) and (4.24). We are left to prove the second estimate $(4.5)_{2}$ on the time-derivates. Given $\tilde{\boldsymbol{v}} \in L^{2}\left(0, t_{*} ; \mathbf{H}\right)$ with $\|\tilde{\boldsymbol{v}}\|_{L^{2}\left(0, t_{*} ; \mathbf{H}\right)} \leq 1$, from $\left(\mathbf{P}_{\varepsilon}\right)_{1}$ we infer that

$$
\left|\left(\partial_{t} \boldsymbol{v}^{\varepsilon}, \tilde{\boldsymbol{v}}\right)\right| \leq C\left[\left\|\boldsymbol{v}^{\varepsilon}\right\|_{H^{2}}|\tilde{\boldsymbol{v}}|+\left\|\boldsymbol{v}^{\varepsilon}\right\|\left\|\boldsymbol{v}^{\varepsilon}\right\|_{H^{2}}|\tilde{\boldsymbol{v}}|+\left\|\theta^{\varepsilon}\right\||\tilde{\boldsymbol{v}}|+\left|\boldsymbol{v}^{\varepsilon}\left\|\tilde{\boldsymbol{v}}|+| \boldsymbol{S}_{\boldsymbol{v}}\right\| \tilde{\boldsymbol{v}}\right|\right] .
$$

Integrating in time on $\left(0, t_{*}\right)$ and using Young's inequality then gives

$$
\partial_{t} \boldsymbol{v}^{\varepsilon} \in L^{2}\left(0, t_{*} ; \mathbf{H}\right),
$$

thanks to the estimate $(4.5)_{1}$. Regarding $\partial_{t} \theta^{\varepsilon}$, we take a test function $\tilde{\theta} \in L^{2}\left(0, t_{*} ; H^{1}\right)$ with norm at most 1 . Thanks to (3.27) and (3.28), we obtain from $\left(\mathbf{P}_{\varepsilon}\right)_{2}$ that

$$
\begin{aligned}
\left|\left\langle\partial_{t} \theta^{\varepsilon}, \tilde{\theta}\right\rangle\right| & \leq C\left[\left\|\theta^{\varepsilon}\right\|\|\tilde{\theta}\|+\left\|\boldsymbol{v}^{\varepsilon}\right\|^{1 / 2}\left\|\boldsymbol{v}^{\varepsilon}\right\|_{H^{2}}^{1 / 2}\left|\theta^{\varepsilon}\right|^{1 / 2}\left\|\theta^{\varepsilon}\right\|^{1 / 2}\|\tilde{\theta}\|+\left\|\boldsymbol{v}^{\varepsilon}\right\||\tilde{\theta}|+\left|S_{\theta} \| \tilde{\theta}\right|\right] \\
& \leq C\left[\left\|\theta^{\varepsilon}\right\|^{2}\left(1+\left|\theta^{\varepsilon}\right|^{2}\right)+\left\|\boldsymbol{v}^{\varepsilon}\right\|^{2}\left(1+\left\|\boldsymbol{v}^{\varepsilon}\right\|_{H^{2}}^{2}\right)+\left|S_{\theta}\right|^{2}+\|\tilde{\theta}\|^{2}\right] .
\end{aligned}
$$

Similarly as for $\partial_{t} \boldsymbol{v}^{\varepsilon}$, we integrate in time on $\left(0, t_{*}\right)$ and use Young's inequality; we arrive at

$$
\partial_{t} \theta^{\varepsilon} \in L^{2}\left(0, t_{*} ;\left(H^{1}\right)^{\prime}\right) .
$$


The argument for $\partial_{t} q^{\varepsilon}$ can be repeated word for word, allowing us to conclude the estimates $(4.5)_{2}$. As we have already observed, all these estimates are actually implemented using a Galerkin method based on the eigenvectors (4.6) of $A$, the proof of Theorem 4.1 is thus concluded.

\section{LOCAL EXISTENCE OF QUASI-STRONG SOLUTIONS}

We provide here a proof of the local existence of quasi-strong solutions of the $(\boldsymbol{v}, \theta, q)$ system by passing to the limit in the approximated problem $\left(\mathbf{P}_{\varepsilon}\right)$ as $\varepsilon \rightarrow 0$. The treatment of the potential temperature and the specific humidity equations resembles that of [6]. As a straightforward consequence, we deduce a local existence result for the $(\boldsymbol{v}, T, q)$ system as well.

5.1. Passage to the limit as $\varepsilon \rightarrow 0$. Thanks to the fact that the estimates (4.5) in Theorem 4.1 are uniform in $\varepsilon$ (the bounds are independent of $\varepsilon$ ), we infer the existence of a triplet $(\boldsymbol{v}, \theta, q)$ such that

$$
\boldsymbol{v} \in C\left(\left[0, t_{*}\right) ; \mathbf{V}\right) \cap L^{2}\left(0, t_{*} ; H^{2}\right), \quad \partial_{t} \boldsymbol{v} \in L^{2}\left(0, t_{*} ; \mathbf{H}\right),
$$

and

$$
\theta, q \in C\left(\left[0, t_{*}\right) ; L^{2}\right) \cap L^{2}\left(0, t_{*} ; H^{1}\right), \quad \partial_{t} \theta, \partial_{t} q \in L^{2}\left(0, t_{*} ;\left(H^{1}\right)^{\prime}\right),
$$

for which the following convergences up to not relabeled subsequences are true. As customary, $\rightarrow, \rightarrow$, and $*$ indicate strong, weak, and weak-* convergence as $\varepsilon \rightarrow 0$, respectively:

- $\boldsymbol{v}^{\varepsilon} \rightarrow \boldsymbol{v}$ in $L^{2}\left(0, t_{*} ; H^{2}\right)$ and $\partial_{t} \boldsymbol{v}^{\varepsilon} \rightarrow \partial_{t} \boldsymbol{v}$ in $L^{2}\left(0, t_{*} ; \mathbf{H}\right)$. As a consequence (see $[22]), \boldsymbol{v}^{\varepsilon} \rightarrow \boldsymbol{v}$ in $L^{2}\left(0, t_{*} ; H^{3 / 2}\right)$.

- $\omega^{\varepsilon} \rightarrow \omega$ in $L^{2}\left(0, t_{*} ; H^{1 / 2}\right)$, which follows from the expression (3.8) for $\omega$.

- $\left(\theta^{\varepsilon}, q^{\varepsilon}\right) \rightarrow(\theta, q)$ in $L^{2}\left(0, t_{*} ; H^{1} \times H^{1}\right)$ and $\partial_{t}\left(\theta^{\varepsilon}, q^{\varepsilon}\right) \rightarrow \partial_{t}(\theta, q)$ in $L^{2}\left(0, t_{*} ;\left(H^{1}\right)^{\prime} \times\right.$ $\left.\left(H^{1}\right)^{\prime}\right)$. Therefore, $\left(\theta^{\varepsilon}, q^{\varepsilon}\right) \rightarrow(\theta, q)$ in $L^{2}\left(0, t_{*} ; L^{2} \times L^{2}\right)$.

- $H_{\varepsilon}\left(q^{\varepsilon}-q_{s}\right) \stackrel{*}{\rightarrow} h_{q}$ in $L^{\infty}\left(\mathcal{M} \times\left[0, t_{*}\right]\right)$.

By interpolation, we also have $\boldsymbol{v} \in C\left(\left[0, t_{*}\right) ; \mathbf{V}\right)$. We now consider test functions of the form $(\tilde{\boldsymbol{v}}, \tilde{\theta}, \tilde{q}) \varphi(t)$, where $(\tilde{\boldsymbol{v}}, \tilde{\theta}, \tilde{q}) \in \mathbf{H} \times H^{1} \times H^{1}$ and $\varphi$ in $C^{1}\left(\left[0, t_{*}\right]\right)$ is a scalar function such that $\varphi\left(t_{*}\right)=0$. We take the $L^{2}$-scalar product for $\left(\mathbf{P}_{\varepsilon}\right)$ with $(\tilde{\boldsymbol{v}}, \tilde{\theta}, \tilde{q}) \varphi(t)$, integrate in time from 0 to $t_{*}$ and integrate by parts for the first term, to arrive at

$$
\begin{aligned}
\int_{0}^{t_{*}} & -\left\langle\boldsymbol{v}^{\varepsilon}, \tilde{\boldsymbol{v}}\right\rangle \partial_{t} \varphi+\left(a_{\boldsymbol{v}}\left(\boldsymbol{v}^{\varepsilon}, \tilde{\boldsymbol{v}}\right)+b\left(\boldsymbol{v}^{\varepsilon}, \boldsymbol{v}^{\varepsilon}, \tilde{\boldsymbol{v}}\right)+e_{p}\left(\theta^{\varepsilon}, \tilde{\boldsymbol{v}}\right)+e_{c}\left(\boldsymbol{v}^{\varepsilon}, \tilde{\boldsymbol{v}}\right)\right) \varphi \mathrm{d} t \\
& =\left\langle\boldsymbol{v}_{0}, \tilde{\boldsymbol{v}}\right\rangle \varphi(0)+\int_{0}^{t_{*}}\left(\boldsymbol{S}_{\boldsymbol{v}}, \tilde{\boldsymbol{v}}\right) \varphi \mathrm{d} t \\
\int_{0}^{t_{*}} & -\left\langle\theta^{\varepsilon}, \tilde{\theta}\right\rangle \partial_{t} \varphi+\left(a_{\theta}\left(\theta^{\varepsilon}, \tilde{\theta}\right)+b\left(\boldsymbol{v}^{\varepsilon}, \theta^{\varepsilon}, \tilde{\theta}\right)+m_{\theta}\left(\theta^{\varepsilon}, \tilde{\theta}\right)\right) \varphi \mathrm{d} t \\
& =\left\langle\theta_{0}, \tilde{\theta}\right\rangle \varphi(0)+\int_{0}^{t_{*}}\left(\left(\frac{L}{c_{p}}\left(\frac{p_{0}}{p}\right)^{\frac{R}{c_{p}}} D_{\varepsilon}\left(\omega^{\varepsilon}, \theta^{\varepsilon}, q^{\varepsilon}\right), \tilde{\theta}\right)+\left(S_{\theta}, \tilde{\theta}\right)\right) \varphi \mathrm{d} t \\
\int_{0}^{t_{*}}-\left\langle q^{\varepsilon}, \tilde{q}\right\rangle \partial_{t} \varphi+\left(a_{q}\left(q^{\varepsilon}, \tilde{q}\right)+b\left(\boldsymbol{v}^{\varepsilon}, q^{\varepsilon}, \tilde{q}\right)\right) \varphi \mathrm{d} t & \\
& =\left\langle q_{0}, \tilde{q}\right\rangle \varphi(0)+\int_{0}^{t_{*}}\left(-\left(D_{\varepsilon}\left(\omega^{\varepsilon}, \theta^{\varepsilon}, q^{\varepsilon}\right), \tilde{q}\right)+\left(S_{q}, \tilde{q}\right)\right) \varphi \mathrm{d} t
\end{aligned}
$$


The only problematic terms are the nonlinear ones, as the linear terms converge to their corresponding limits in a straightforward manner due to the above convergences. We start with the term

We have

$$
\int_{0}^{t_{*}}\left(\frac{L}{c_{p}}\left(\frac{p_{0}}{p}\right)^{\frac{R}{c_{p}}} D_{\varepsilon}\left(\omega^{\varepsilon}, \theta^{\varepsilon}, q^{\varepsilon}\right), \tilde{\theta}\right) \varphi \mathrm{d} t
$$

$$
\begin{aligned}
\int_{0}^{t_{*}} & \left(\frac{L}{c_{p}}\left(\frac{p_{0}}{p}\right)^{\frac{R}{c_{p}}} D_{\varepsilon}\left(\omega^{\varepsilon}, \theta^{\varepsilon}, q^{\varepsilon}\right), \tilde{\theta}\right) \varphi \mathrm{d} t-\int_{0}^{t_{*}}\left(\frac{L}{c_{p}}\left(\frac{p_{0}}{p}\right)^{\frac{R}{c_{p}}} D\left(\omega, \theta, h_{q}\right), \tilde{\theta}\right) \varphi \mathrm{d} t \\
= & \int_{0}^{t_{*}}\left(\frac{L}{c_{p}}\left(\frac{p_{0}}{p}\right)^{\frac{R}{c_{p}}} \omega^{\varepsilon-} H_{\varepsilon}\left(q^{\varepsilon}-q_{s}\right)\left(\widetilde{F}\left(p, \theta^{\varepsilon}\right)-\widetilde{F}(p, \theta)\right), \tilde{\theta}\right) \varphi \mathrm{d} t \\
& +\int_{0}^{t_{*}}\left(\frac{L}{c_{p}}\left(\frac{p_{0}}{p}\right)^{\frac{R}{c_{p}}}\left(\omega^{\varepsilon-}-\omega^{-}\right) H_{\varepsilon}\left(q^{\varepsilon}-q_{s}\right) \widetilde{F}(p, \theta), \tilde{\theta}\right) \varphi \mathrm{d} t \\
& +\int_{0}^{t_{*}}\left(\frac{L}{c_{p}}\left(\frac{p_{0}}{p}\right)^{\frac{R}{c_{p}}} \omega^{-}\left(H_{\varepsilon}\left(q^{\varepsilon}-q_{s}\right)-h_{q}\right) \widetilde{F}(p, \theta), \tilde{\theta}\right) \varphi \mathrm{d} t \\
& =J_{1}+J_{2}+J_{3} .
\end{aligned}
$$

Using the boundedness of $H_{\varepsilon}$ (see (4.2)) and the Lipschitz condition on $\widetilde{F}(p, \cdot)$ (see (3.4)), we bound the term $J_{1}$ as

$$
\begin{aligned}
\left|J_{1}\right| & \leq c \int_{0}^{t_{*}} \int_{\mathcal{M}}\left|\omega^{\varepsilon}\left\|\theta^{\varepsilon}-\theta\right\| \tilde{\theta}\right| \mathrm{d} \mathcal{M} \mathrm{d} t \\
& \leq c \int_{0}^{t_{*}}\left\|\omega^{\varepsilon}(t)\right\|_{L^{3}}\left|\theta^{\varepsilon}(t)-\theta(t)\right|\|\tilde{\theta}\|_{L^{6}} \mathrm{~d} t \\
& \leq c\|\tilde{\theta}\| \int_{0}^{t_{*}}\left\|\boldsymbol{v}^{\varepsilon}\right\|_{H^{2}}\left|\theta^{\varepsilon}(t)-\theta(t)\right| \mathrm{d} t \\
& \leq c\left\|\boldsymbol{v}^{\varepsilon}\right\|_{L^{2}\left(0, t_{*} ; H^{2}\right)}\left\|\theta^{\varepsilon}-\theta\right\|_{L^{2}\left(0, t_{*} ; L^{2}\right)}\|\tilde{\theta}\|,
\end{aligned}
$$

which converges to 0 as $\varepsilon \rightarrow 0$ thanks to the boundedness of $\boldsymbol{v}^{\varepsilon}$ and the strong convergence of $\theta^{\varepsilon}$. As a preliminary, using the Sobolev embedding $H^{1 / 2} \subset L^{3}$, we obtain

$$
\omega^{\varepsilon} \rightarrow \omega \text {, strongly in } L^{2}\left(0, t_{*} ; L^{3}\right),
$$

and thus also in $L^{2}\left(0, t_{*} ; L^{2}\right)$. Now, for the second term $J_{2}$, using the boundedness of $H_{\varepsilon}$ and $\widetilde{F}$ (see (4.2) and (3.4)), we obtain

$$
\left|J_{2}\right| \leq c \int_{0}^{t_{*}} \int_{\mathcal{M}}\left|\omega^{\varepsilon-}-\omega^{-}\right||\tilde{\theta}| \mathrm{d} \mathcal{M} \mathrm{d} t \leq c|| \omega^{\varepsilon-}-\omega^{-} \|_{L^{2}\left(0, t_{*} ; H\right)}|\tilde{\theta}|
$$

which converges to 0 as $\varepsilon \rightarrow 0$ by using (5.2). For the last term $J_{3}$, we observe that $\omega^{-} \widetilde{F}(p, \theta) \tilde{\theta} \varphi$ belongs to $L^{1}\left(\mathcal{M} \times\left(0, t_{*}\right)\right)$. Hence, the weak-* convergence of $H_{\varepsilon}\left(q_{\varepsilon}-q_{s}\right)$ is enough to pass to the limit. Therefore, as $\varepsilon \rightarrow 0$,

$$
\int_{0}^{t_{*}}\left(\frac{L}{c_{p}}\left(\frac{p_{0}}{p}\right)^{\frac{R}{c_{p}}} D_{\varepsilon}\left(\omega^{\varepsilon}, \theta^{\varepsilon}, q^{\varepsilon}\right), \tilde{\theta}\right) \varphi \mathrm{d} t \rightarrow \int_{0}^{t_{*}}\left(\frac{L}{c_{p}}\left(\frac{p_{0}}{p}\right)^{\frac{R}{c_{p}}} D\left(\omega, \theta, h_{q}\right), \tilde{\theta}\right) \varphi \mathrm{d} t .
$$

The analogous term in the $q$-equation converges in the same exact way. 
We now turn to the trilinear term $b$. Considering the typical most problematic term, we have that, as $\varepsilon \rightarrow 0$,

$$
\int_{0}^{t_{*}} \int_{\mathcal{M}} \omega^{\varepsilon} \partial_{p} \boldsymbol{v}^{\varepsilon} \tilde{\boldsymbol{v}} \varphi \mathrm{d} \mathcal{M} \mathrm{d} t \rightarrow \int_{0}^{t_{*}} \int_{\mathcal{M}} \omega \partial_{p} \boldsymbol{v} \tilde{\boldsymbol{v}} \varphi \mathrm{d} \mathcal{M} \mathrm{d} t
$$

where we used (5.2) and that $\partial_{p} \boldsymbol{v}^{\varepsilon}$ converges weakly to $\partial_{p} \boldsymbol{v}$ in $L^{2}\left(0, t_{*} ; L^{6}\right)$ by the Sobolev embedding $H^{1} \subset L^{6}$. The other terms in $b$ are similar or simpler. Therefore, we conclude that, as $\varepsilon \rightarrow 0$,

$$
\int_{0}^{t_{*}} b\left(\boldsymbol{v}^{\varepsilon}, \boldsymbol{v}^{\varepsilon}, \tilde{\boldsymbol{v}}\right) \varphi \mathrm{d} t \rightarrow \int_{0}^{t_{*}} b(\boldsymbol{v}, \boldsymbol{v}, \tilde{\boldsymbol{v}}) \varphi \mathrm{d} t
$$

We are left with the trilinear form $b$ involving $\theta$ (the one involving $q$ is exactly the same). Similarly, we consider the typical most problematic term, and hence, it is enough to show that

We have

$$
\int_{0}^{t_{*}} \int_{\mathcal{M}} \omega^{\varepsilon} \partial_{p} \theta^{\varepsilon} \tilde{\theta} \varphi \mathrm{d} \mathcal{M} \mathrm{d} t \rightarrow \int_{0}^{t_{*}} \int_{\mathcal{M}} \omega \partial_{p} \theta \tilde{\theta} \varphi \mathrm{d} \mathcal{M} \mathrm{d} t
$$

$$
\begin{aligned}
\int_{0}^{t_{*}} & \int_{\mathcal{M}}\left(\omega^{\varepsilon} \partial_{p} \theta^{\varepsilon}-\omega \partial_{p} \theta\right) \tilde{\theta} \varphi \mathrm{d} \mathcal{M} \mathrm{d} t \\
\quad= & \int_{0}^{t_{*}} \int_{\mathcal{M}}\left(\omega^{\varepsilon}-\omega\right) \partial_{p} \theta^{\varepsilon} \tilde{\theta} \varphi \mathrm{d} \mathcal{M} \mathrm{d} t+\int_{0}^{t_{*}} \int_{\mathcal{M}} \omega\left(\partial_{p} \theta^{\varepsilon}-\partial_{p} \theta\right) \tilde{\theta} \varphi \mathrm{d} \mathcal{M} \mathrm{d} t
\end{aligned}
$$

with

$$
\begin{aligned}
\left|\int_{0}^{t_{*}} \int_{\mathcal{M}}\left(\omega^{\varepsilon}-\omega\right) \partial_{p} \theta^{\varepsilon} \tilde{\theta} \varphi \mathrm{d} \mathcal{M} \mathrm{d} t\right| & \leq c \int_{0}^{t_{*}}\left\|\omega^{\varepsilon}-\omega\right\|_{L^{3}}\left|\partial_{p} \theta^{\varepsilon}\right|\|\tilde{\theta}\| \mathrm{d} t \\
& \leq c\left\|\omega^{\varepsilon}-\omega\right\|_{L^{2}\left(0, t_{*} ; L^{3}\right)}\left\|\theta^{\varepsilon}\right\|_{L^{2}\left(0, t_{*} ; H^{1}\right)}\|\tilde{\theta}\| \rightarrow 0 \quad(\varepsilon \rightarrow 0),
\end{aligned}
$$

and

$$
\left|\int_{0}^{t_{*}} \int_{\mathcal{M}} \omega\left(\partial_{p} \theta^{\varepsilon}-\partial_{p} \theta\right) \tilde{\theta} \varphi \mathrm{d} \mathcal{M} \mathrm{d} t\right| \rightarrow 0 \quad(\varepsilon \rightarrow 0)
$$

as $\partial_{p} \theta^{\varepsilon} \rightarrow \partial_{p} \theta$ in $L^{2}\left(0, t_{*} ; L^{2}\right)$ and $\omega \tilde{\theta} \varphi \in L^{2}\left(0, t_{*} ; L^{2}\right)$. Indeed, $\omega \in L^{2}\left(0, t_{*} ; L^{3}\right)$ and $\tilde{\theta} \varphi \in L^{\infty}\left(0, t_{*} ; L^{6}\right)$.

It remains to show that $h_{q}$ belongs to $H\left(q-q_{s}\right)$ in the weak sense specified by the variational inequality (3.32). This has been already proved in [6], but we sketch the argument here for the sake of completeness. For every $\varepsilon>0$, we have an approximate variational inequality

$$
\int_{0}^{t_{*}}\left(K_{\varepsilon}\left(\tilde{q}-q_{s}\right), 1\right) \mathrm{d} t-\int_{0}^{t_{*}}\left(K_{\varepsilon}\left(q_{\varepsilon}-q_{s}\right), 1\right) \mathrm{d} t \geq \int_{0}^{t_{*}}\left\langle H_{\varepsilon}\left(q_{\varepsilon}-q_{s}\right), \tilde{q}-q_{\varepsilon}\right\rangle \mathrm{d} t
$$

for each $\tilde{q} \in L^{2}\left(0, t_{*} ; H^{1}\right)$, since $H_{\varepsilon}\left(q_{\varepsilon}-q_{s}\right)$ is the Gâteaux derivative of the convex function

$$
\int_{0}^{t_{*}}\left(K_{\varepsilon}(\cdot), 1\right) \mathrm{d} t: L^{2}\left(0, t_{*} ; V\right) \rightarrow \mathbb{R}
$$

at the point $q_{\varepsilon}-q_{s}$ (see (4.1)). From the weak-* convergence $H_{\varepsilon}\left(q_{\varepsilon}-q_{s}\right) \stackrel{*}{\rightarrow} h_{q}$ in $L^{\infty}\left(\mathcal{M} \times\left[0, t_{*}\right]\right)$ and the strong convergence $q_{\varepsilon} \rightarrow q$ in $L^{2}\left(0, t_{*} ; H\right)$ we find that

$$
\int_{0}^{t_{*}}\left\langle H_{\varepsilon}\left(q_{\varepsilon}-q_{s}\right), q_{\varepsilon}-\tilde{q}\right\rangle \mathrm{d} t \rightarrow \int_{0}^{t_{*}}\left\langle h_{q}, q-\tilde{q}\right\rangle \mathrm{d} t, \quad \forall \tilde{q} \in L^{2}\left(0, t_{*} ; V\right)
$$


as $\varepsilon \rightarrow 0$. Moreover, owing to (4.3) and (4.4), we observe that

$$
\begin{aligned}
& \left|\int_{0}^{t_{*}}\left(K_{\varepsilon}\left(q_{\varepsilon}-q_{s}\right), 1\right) \mathrm{d} t-\int_{0}^{t_{*}}\left(\left[q-q_{s}\right]^{+}, 1\right) \mathrm{d} t\right| \\
& \quad \leq \int_{0}^{t_{*}}\left(\left|K_{\varepsilon}\left(q_{\varepsilon}-q_{s}\right)-K_{\varepsilon}\left(q-q_{s}\right)\right|, 1\right) \mathrm{d} t+\int_{0}^{t_{*}}\left(\left|K_{\varepsilon}\left(q-q_{s}\right)-\left[q-q_{s}\right]^{+}\right|, 1\right) \mathrm{d} t \\
& \quad \leq|\mathcal{M}|^{1 / 2} t_{*}^{1 / 2}\left\|q_{\varepsilon}-q\right\|_{L^{2}\left(0, t_{*} ; H\right)}+\frac{\varepsilon}{2}|\mathcal{M}| t_{*} .
\end{aligned}
$$

Therefore,

$$
\lim _{\varepsilon \rightarrow 0} \int_{0}^{t_{*}}\left(K_{\varepsilon}\left(q_{\varepsilon}-q_{s}\right), 1\right) \mathrm{d} t=\int_{0}^{t_{*}}\left(\left[q-q_{s}\right]^{+}, 1\right) \mathrm{d} t .
$$

From the calculation above, it is also clear that

$$
\lim _{\varepsilon \rightarrow 0} \int_{0}^{t_{*}}\left(K_{\varepsilon}\left(\tilde{q}-q_{s}\right), 1\right) \mathrm{d} t=\int_{0}^{t_{*}}\left(\left[\tilde{q}-q_{s}\right]^{+}, 1\right) \mathrm{d} t, \quad \tilde{q} \in L^{2}\left(0, t_{*} ; V\right) .
$$

Consequently, we can pass to the limit as $\varepsilon \rightarrow 0$ in (5.4), concluding that

$$
\left.\int_{0}^{t_{*}}\left\langle\left[\tilde{q}-q_{s}\right)\right]^{+}, 1\right\rangle \mathrm{d} t-\int_{0}^{t_{*}}\left\langle\left[q-q_{s}\right]^{+}, 1\right\rangle \mathrm{d} t \geq \int_{0}^{t_{*}}\left\langle h_{q}, \tilde{q}-q\right\rangle \mathrm{d} t, \quad \forall \tilde{q} \in L^{2}\left(0, t_{*} ; V\right) .
$$

Again, this implies in particular that

$$
\left(\left[\tilde{q}-q_{s}\right]^{+}, 1\right)-\left(\left[q-q_{s}\right]^{+}, 1\right) \geq\left\langle h_{q}, \tilde{q}-q\right\rangle,
$$

for every $\tilde{q} \in V$ and a.e. $t \in\left(0, t_{*}\right]$, as desired. We have proved the following statement.

Theorem 5.1. Let $\boldsymbol{v}_{0} \in \mathbf{V}$ and $\theta_{0}, q_{0} \in L^{2}$. Assume $\left(\boldsymbol{S}_{\boldsymbol{v}}, S_{\theta}, S_{q}\right) \in L^{2}\left(0, t_{1} ; H\right), q_{s} \in(0,1)$ and $t_{1}>0$. There exists $t_{*}>0\left(t_{*} \leq t_{1}\right)$ and a quasi-strong solution to (3.11)-(3.19) such that

$$
\begin{array}{ll}
\boldsymbol{v} \in C\left(\left[0, t_{*}\right) ; \mathbf{V}\right) \cap L^{2}\left(0, t_{*} ; H^{2}\right), & \theta, q \in C\left(\left[0, t_{*}\right) ; L^{2}\right) \cap L^{2}\left(0, t_{*} ; H^{1}\right), \\
\partial_{t} \boldsymbol{v} \in L^{2}\left(0, t_{*} ; \mathbf{H}\right), & \partial_{t} \theta, \partial_{t} q \in L^{2}\left(0, t_{*} ;\left(H^{1}\right)^{\prime}\right) .
\end{array}
$$

Remark 5.2. By Lemma 4.3, the quasi-strong solution $(\boldsymbol{v}, \theta, q)$ to (3.11)-(3.13) satisfies the estimate

$$
\|(\boldsymbol{v}, \theta, q)\|_{L^{\infty}\left(0, t_{*} ; H\right)}^{2}+\|(\boldsymbol{v}, \theta, q)\|_{L^{2}\left(0, t_{*} ; V\right)}^{2} \leq C e^{C t_{1}}\left(\left|\left(\boldsymbol{v}_{0}, \theta_{0}, q_{0}\right)\right|+\left\|\left(\boldsymbol{S}_{\boldsymbol{v}}, S_{\theta}, S_{q}\right)\right\|_{L^{2}\left(0, t_{1} ; H\right)}^{2}\right),
$$

which shows that we have a uniform bound for $\|(\boldsymbol{v}, \theta, q)\|_{H}$. This estimate will be very useful for obtaining the global strong solutions.

5.2. The $(\boldsymbol{v}, T, q)$-system. We now revert back to our original $(\boldsymbol{v}, T, q)$-system. The weak formulation reads

$$
\begin{aligned}
& \left\langle\partial_{t} \boldsymbol{v}, \tilde{\boldsymbol{v}}\right\rangle+a_{\boldsymbol{v}}(\boldsymbol{v}, \tilde{\boldsymbol{v}})+b(\boldsymbol{v}, \boldsymbol{v}, \tilde{\boldsymbol{v}})+e_{p}(T, \tilde{\boldsymbol{v}})+e_{c}(\boldsymbol{v}, \tilde{\boldsymbol{v}})=\left(\boldsymbol{S}_{\boldsymbol{v}}, \tilde{\boldsymbol{v}}\right), \\
& \left\langle\partial_{t} T, \widetilde{T}\right\rangle+a_{T}(T, \widetilde{T})+b(\boldsymbol{v}, T, \widetilde{T})=m_{T}(\omega, T, \widetilde{T})+\frac{L}{c_{p}}\left(D\left(\omega, T, h_{q}\right), \widetilde{T}\right)+\left(S_{T}, \widetilde{T}\right), \\
& \left\langle\partial_{t} q, \tilde{q}\right\rangle+a_{q}(q, \tilde{q})+b(\boldsymbol{v}, q, \tilde{q})=-\left(D\left(\omega, \theta, h_{q}\right), \tilde{q}\right)+\left(S_{q}, \tilde{q}\right),
\end{aligned}
$$

where

$$
m_{T}(\omega, T, \widetilde{T})=\int_{\mathcal{M}} \frac{R c_{p}}{p} \omega T \widetilde{T} \mathrm{~d} \mathcal{M}
$$


In order not to complicate the notation, we redefine

$$
e_{p}(T, \tilde{\boldsymbol{v}})=\int_{\mathcal{M}} \nabla \int_{p}^{p_{1}} \frac{R}{p^{\prime}} T \mathrm{~d} p^{\prime} \cdot \tilde{\boldsymbol{v}} d \mathcal{M}
$$

and

$$
D\left(\omega, T, h_{q}\right)=\frac{1}{p} \omega^{-} h_{q} F(T) .
$$

Using Theorem 5.1 and Remark 5.2 with the relation (3.1) between $T$ and $\theta$, we obtain the following.

Corollary 5.3. Let there be given $\boldsymbol{v}_{0} \in \mathbf{V}$ and $T_{0}, q_{0} \in L^{2}$. Assume $\left(\boldsymbol{S}_{\boldsymbol{v}}, S_{T}, S_{q}\right) \in$ $L^{2}\left(0, t_{1} ; H\right), q_{s} \in(0,1)$ and $t_{1}>0$. There exists $t_{*}>0\left(t_{*} \leq t_{1}\right)$ and a quasi-strong solution to (5.5)-(5.7) such that

$$
\begin{array}{ll}
\boldsymbol{v} \in C\left(\left[0, t_{*}\right) ; \mathbf{V}\right) \cap L^{2}\left(0, t_{1} ; H^{2}\right), & T, q \in C\left(\left[0, t_{*}\right) ; L^{2}\right) \cap L^{2}\left(0, t_{*} ; H^{1}\right), \\
\partial_{t} \boldsymbol{v} \in L^{2}\left(0, t_{*} ; \mathbf{H}\right), & \partial_{t} T, \partial_{t} q \in L^{2}\left(0, t_{*} ;\left(H^{1}\right)^{\prime}\right),
\end{array}
$$

and the following $L^{2}$-estimate

$\|(\boldsymbol{v}, T, q)\|_{L^{\infty}\left(0, t_{*} ; H\right)}^{2}+\|(\boldsymbol{v}, T, q)\|_{L^{2}\left(0, t_{*} ; V\right)}^{2} \leq C e^{C t_{1}}\left(\left|\left(\boldsymbol{v}_{0}, T_{0}, q_{0}\right)\right|+\left\|\left(\boldsymbol{S}_{\boldsymbol{v}}, S_{T}, S_{q}\right)\right\|_{L^{2}\left(0, t_{1} ; H\right)}^{2}\right)$,

holds for some constant $C>0$ independent of initial data and the time $t_{1}$ and $t_{*}$.

\section{Global Strong solutions}

The notion of quasi-strong solutions was introduced in the previous section in order to deal with the lesser regularity of the vertical component $\omega$ of the velocity field with respect to $\boldsymbol{v}$. Moreover, the use of the potential temperature $\theta$ turned out to be convenient to obtain the basic $L^{2}-H^{1}$ estimates, circumventing the difficulty of dealing with the anti-dissipative term

$$
-\frac{R}{c_{p} p} \omega T
$$

present in the equation for the temperature $T$. From here on, we only consider the $(\boldsymbol{v}, T, q)$ system of equations, for which existence of quasi-strong solutions has been established in Corollary 5.3.

The first goal of this section is to prove that, for more regular initial data, the local solutions derived in the previous section are in fact strong. The second goal aims to show the existence of global strong solutions by using Theorem A.1 (see also [20]).

6.1. Strong solutions. We begin by defining the concept of strong solutions.

Definition 6.1. Let $\left(\boldsymbol{v}_{0}, T_{0}, q_{0}\right)$ be given in $V$. Assume $\left(\boldsymbol{S}_{\boldsymbol{v}}, S_{T}, S_{q}\right)$ are given in $L^{2}\left(0, t_{1} ; H\right)$, $q_{s} \in(0,1)$ and $t_{1}>0$. A vector $(\boldsymbol{v}, T, q)$ is a strong solution to (3.11)-(3.19) if

$$
\begin{aligned}
& (\boldsymbol{v}, T, q) \in C\left(\left[0, t_{1}\right] ; V\right) \cap L^{2}\left(0, t_{1} ; W\right), \\
& \partial_{t}(\boldsymbol{v}, T, q) \in L^{2}\left(0, t_{1} ; H\right),
\end{aligned}
$$


and, for almost every $t \in\left[0, t_{1}\right]$ and every $(\tilde{\boldsymbol{v}}, \widetilde{T}, \tilde{q}) \in V$,

$$
\begin{aligned}
& \left\langle\partial_{t} \boldsymbol{v}, \tilde{\boldsymbol{v}}\right\rangle+a_{\boldsymbol{v}}(\boldsymbol{v}, \tilde{\boldsymbol{v}})+b(\boldsymbol{v}, \boldsymbol{v}, \tilde{\boldsymbol{v}})+e_{p}(T, \tilde{\boldsymbol{v}})+e_{c}(\boldsymbol{v}, \tilde{\boldsymbol{v}})=\left(\boldsymbol{S}_{\boldsymbol{v}}, \tilde{\boldsymbol{v}}\right), \\
& \left\langle\partial_{t} T, \widetilde{T}\right\rangle+a_{T}(T, \widetilde{T})+b(\boldsymbol{v}, T, \widetilde{T})=m_{T}(\omega, T, \widetilde{T})+\frac{L}{c_{p}}\left(D\left(\omega, T, h_{q}\right), \widetilde{T}\right)+\left(S_{T}, \widetilde{T}\right), \\
& \left\langle\partial_{t} q, \tilde{q}\right\rangle+a_{q}(q, \tilde{q})+b(\boldsymbol{v}, q, \tilde{q})=-\left(D\left(\omega, T, h_{q}\right), \tilde{q}\right)+\left(S_{q}, \tilde{q}\right),
\end{aligned}
$$

for some $h_{q} \in L^{\infty}\left(\mathcal{M} \times\left(0, t_{1}\right)\right)$ which satisfies the variational inequality

$$
\left(\left[\tilde{q}-q_{s}\right]^{+}, 1\right)-\left(\left[q-q_{s}\right]^{+}, 1\right) \geq\left(h_{q}, \tilde{q}-q\right), \quad \text { a.e. } t \in\left[0, t_{1}\right], \quad \forall \tilde{q} \in H^{1} .
$$

The only difference between the definitions of quasi-strong and strong solutions lies in the regularity required for the triplet $(\boldsymbol{v}, T, q)$.

6.2. Local existence of strong solutions. From Corollary 5.3, given initial data $\boldsymbol{v}_{0} \in$ $\mathbf{V}, T_{0} \in H^{1}$, and $q_{0} \in H^{1}$, we deduce that a quasi-strong solution exists, at least locally in time. The velocity field $\boldsymbol{v}$ already has the regularity required to be a strong solution. Our aim is now to improve the regularity on $T$ and $q$ on the same small time-interval of our local quasi-strong solution. We now prove the following theorem.

Theorem 6.2. Let there be given $\boldsymbol{v}_{0} \in \mathbf{V}$ and $T_{0}, q_{0} \in H^{1}$. Assume $\left(\boldsymbol{S}_{\boldsymbol{v}}, S_{T}, S_{q}\right) \in$ $L^{2}\left(0, t_{1} ; H\right), q_{s} \in(0,1)$, and $t_{1}>0$. Then there exists $t_{*}>0\left(t_{*} \leq t_{1}\right)$ and a strong solution to (5.5)-(5.7) such that

$$
\begin{aligned}
& (\boldsymbol{v}, T, q) \in C\left(\left[0, t_{*}\right) ; V\right) \cap L^{2}\left(0, t_{*} ; W\right), \\
& \partial_{t}(\boldsymbol{v}, T, q) \in L^{2}\left(0, t_{*} ; H\right) .
\end{aligned}
$$

Proof. Let $t_{*}>0$ be the time of existence of a local quasi-strong solution. We start with improving the regularity on $T$, showing that

$$
T \in L^{\infty}\left(0, t_{*} ; H^{1}\right) \cap L^{2}\left(0, t_{*} ; H^{2}\right) .
$$

Testing formally equation (5.6) with $A_{T} T$, assuming that $A_{T} T \in L^{2}\left(0, t_{1} ;\left(H^{1}\right)^{\prime}\right)$, we obtain the differential equation

$$
\begin{aligned}
\frac{1}{2} \frac{\mathrm{d}}{\mathrm{d} t}\left|A_{T}^{1 / 2} T\right|^{2}+\left|A_{T} T\right|^{2}= & -b\left(\boldsymbol{v}, T, A_{T} T\right)+m_{T}\left(\omega, T, A_{T} T\right) \\
& +\frac{L}{c_{p}}\left(D\left(\omega, T, h_{q}\right), A_{T} T\right)+\left(S_{T}, A_{T} T\right) .
\end{aligned}
$$

We now estimate the terms on the right. Thanks to (3.26) written only for $b$, we have

$$
\begin{aligned}
\left|b\left(\boldsymbol{v}, T, A_{T} T\right)\right| & \leq c\|\boldsymbol{v}\|^{1 / 2}\|\boldsymbol{v}\|_{H^{2}}^{1 / 2}\|T\|^{1 / 2}\|T\|_{H^{2}}^{1 / 2}\left|A_{T} T\right| \\
& \leq c\|\boldsymbol{v}\|^{1 / 2}\|\boldsymbol{v}\|_{H^{2}}^{1 / 2}\|T\|^{1 / 2}\left|A_{T} T\right|^{3 / 2} \\
& \leq \frac{1}{8}\left|A_{T} T\right|^{2}+c\|\boldsymbol{v}\|^{2}\|\boldsymbol{v}\|_{H^{2}}^{2}\|T\|^{2}
\end{aligned}
$$

Moreover,

$$
\begin{aligned}
m_{T}\left(\omega, T, A_{T} T\right) & \leq c\|\omega\|_{L^{4}}\|T\|_{L^{4}}\left|A_{T} T\right| \\
& \leq c\|\omega\|\|T\|\left|A_{T} T\right| \\
& \leq \frac{1}{8}\left|A_{T} T\right|^{2}+c\|\boldsymbol{v}\|_{H^{2}}^{2}\|T\|^{2} .
\end{aligned}
$$


The third term is estimated as

$$
\begin{aligned}
\frac{L}{c_{p}}\left(D\left(\omega, T, h_{q}\right), A_{T} T\right) & \leq c\left|\omega^{-} \| A_{T} T\right| \\
& \leq \frac{1}{8}\left|A_{T} T\right|^{2}+c\|\boldsymbol{v}\|^{2},
\end{aligned}
$$

and, lastly,

$$
\left|\left(S_{T}, A_{T} T\right)\right| \leq \frac{1}{8}\left|A_{T} T\right|^{2}+c\left|S_{T}\right|^{2}
$$

Therefore, by equivalence of the norms $\left|A_{T}^{1 / 2} T\right|$ and $\|T\|$, we obtain

$$
\frac{\mathrm{d}}{\mathrm{d} t}\left|A_{T}^{1 / 2} T\right|^{2}+\left|A_{T} T\right|^{2} \leq c\left(\|\boldsymbol{v}\|^{2}\|\boldsymbol{v}\|_{H^{2}}^{2}+\|\boldsymbol{v}\|_{H^{2}}^{2}\right)\left|A_{T}^{1 / 2} T\right|^{2}+c\left(\|\boldsymbol{v}\|^{2}+\left|S_{T}\right|^{2}\right) .
$$

Note that since

$$
\boldsymbol{v} \in C\left(\left[0, t_{*}\right) ; \mathbf{V}\right) \cap L^{2}\left(0, t_{*} ; H^{2}\right)
$$

and

$$
S_{T} \in L^{2}\left(0, t_{*} ; L^{2}\right)
$$

we see that

$$
\|\boldsymbol{v}\|^{2}\|\boldsymbol{v}\|_{H^{2}}^{2}+\|\boldsymbol{v}\|_{H^{2}}^{2} \in L^{1}\left(0, t_{*}\right), \quad\|\boldsymbol{v}\|^{2}+\left|S_{T}\right|^{2} \in L^{1}\left(0, t_{*}\right) .
$$

Therefore, the claim that

$$
T \in L^{\infty}\left(0, t_{*} ; H^{1}\right) \cap L^{2}\left(0, t_{*} ; H^{2}\right)
$$

may be deduced from an application of the Gronwall lemma and the implementation of a Galerkin method. Once this is settled, the fact that

$$
\partial_{t} T \in L^{2}\left(0, t_{*} ; L^{2}\right)
$$

is deduced directly from equation (5.6). This implies, in particular, that

$$
T \in C\left(\left[0, t_{*}\right) ; H^{1}\right) \cap L^{2}\left(0, t_{*} ; H^{2}\right),
$$

concluding the proof. The regularity of $q$ can be established in the same way, as the $q$-equation involves the same terms except for the trilinear form $m_{T}$. Theorem 6.2 is then proven.

6.3. Global existence of strong solutions. The existence of global strong solutions for the full primitive equations with saturation (2.11)-(2.16) with initial and boundary conditions (2.24)-(2.27) (or the reformulated version (5.5)-(5.7)) is a direct consequence of Theorem A.1 from the Appendix and Theorem 6.2.

Theorem 6.3. Let there be given $\boldsymbol{v}_{0} \in \mathbf{V}$ and $T_{0}, q_{0} \in H^{1}$. Assume $\left(\boldsymbol{S}_{\boldsymbol{v}}, S_{T}, S_{q}\right) \in$ $L^{2}\left(0, t_{1} ; H\right), q_{s} \in(0,1)$ and $t_{1}>0$. Then there exists a global strong solution to (5.5)(5.7) such that

$$
\begin{aligned}
& (\boldsymbol{v}, T, q) \in C\left(\left[0, t_{1}\right] ; \mathbf{V}\right) \cap L^{2}\left(0, t_{1} ; W\right), \\
& \partial_{t}(\boldsymbol{v}, T, q) \in L^{2}\left(0, t_{1} ; H\right) .
\end{aligned}
$$


Proof. From Theorem 6.2, we already know the existence of a local strong solutions in some maximum time interval $\left[0, t_{*}\right)\left(t_{*} \leq t_{1}\right)$. Therefore, in order to find the global strong solution, it is enough to show that the uniform bound $\|(\boldsymbol{v}, T, q)(\cdot, t)\| \leq \widetilde{M}$ independent of $t \in\left[0, t_{*}\right)$, which implies that no blow-up can occur at the time $t=t_{*}$.

Let us first rewrite the original velocity equation (3.11) as

$$
\frac{\partial \boldsymbol{v}}{\partial t}+\boldsymbol{v} \cdot \nabla \boldsymbol{v}+\omega \frac{\partial \boldsymbol{v}}{\partial p}+\nabla \Phi_{s}+\mathcal{A}_{\boldsymbol{v}} \boldsymbol{v}=\boldsymbol{S}_{\boldsymbol{v}}-f \boldsymbol{k} \times \boldsymbol{v}-\nabla \int_{p}^{p_{1}} \frac{R}{p^{\prime}} T \mathrm{~d} p^{\prime}=: \overline{\boldsymbol{S}}_{\boldsymbol{v}}
$$

Then by the $L^{2}$-uniform estimate (5.8) in Corollary 5.3, we deduce

$$
\left\|\overline{\boldsymbol{S}}_{\boldsymbol{v}}\right\|_{L^{2}\left(0, t_{*} ; L^{2}\right)} \leq \widetilde{M}
$$

for some $\widetilde{M}>0$ independent of $t_{*}$. Therefore, applying Theorem A.1 to (6.5) with $\boldsymbol{S}_{\boldsymbol{v}}=\overline{\boldsymbol{S}}_{\boldsymbol{v}}$, we obtain

$$
\|\boldsymbol{v}(t)\|+\|\boldsymbol{v}\|_{L^{2}\left(0, t_{*} ; H^{2}\right)} \leq \widetilde{M}, \quad \text { independent of } t_{*} .
$$

With the uniform estimate (6.6) for $\boldsymbol{v}$ at hand, proceeding exactly as in the proof of Theorem 6.2 to seek the uniform $H^{1}$-estimate for $T$ and $q$, we are able to obtain the uniform bound for $\|(T, q)(\cdot, t)\|_{V}$. In conclusion, we have

$$
\sup _{t \in\left[0, t_{*}\right)}\|(\boldsymbol{v}, T, q)(t)\|+\|(\boldsymbol{v}, T, q)\|_{L^{2}\left(0, t_{*} ; H^{2}\right)} \leq \widetilde{M},
$$

for some $\widetilde{M}>0$ independent of $t_{*}$. We thus completed the proof of the existence of global strong solutions to (5.5)-(5.7), that is, the proof of Theorem 6.3.

\section{UNIQUENESS OF STRONG AND QUASI-STRONG SOLUTIONS}

Here we prove uniqueness and continuous dependence results for the quasi-strong solutions to (5.5)-(5.7). As a straightforward consequence, strong solutions turn out to be unique as well. The proof combines many ideas. The velocity equations are treated as in $[4,11,38]$, while the temperature equation has to be substituted by the moist static energy equation in order to exploit a certain cancellation property, as in [5]. Finally, tools from monotone operator theory and variational inequalities $[2,8,16,31]$ turn out to be useful to handle the specific humidity equation, again following along the lines in [5]. The following is the main result of this section.

Theorem 7.1. Assume that $\left(\boldsymbol{v}_{1}, T_{1}, q_{1}\right)$ and $\left(\boldsymbol{v}_{2}, T_{2}, q_{2}\right)$ are two (strong or) quasi-strong solutions to $(5.5)-(5.7)$ on $\left[0, t_{1}\right]$, with the nonlinear function $F$ replaced by its positive part $F^{+}$, and with initial data $\boldsymbol{v}_{i}^{0} \in \mathbf{V}$, an $T_{i}^{0}, q_{i}^{0} \in L^{2}$, for $i=1,2$. Then, there exists positive constants $c$ and $c_{0}=c_{0}\left(\left\|\boldsymbol{v}_{i}^{0}\right\|,\left|T_{i}^{0}\right|,\left|q_{i}^{0}\right|\right)$ such that

$$
\sup _{t \in\left[0, t_{1}\right]}\left\|\left(\boldsymbol{v}_{1}, T_{1}, q_{1}\right)-\left(\boldsymbol{v}_{2}, T_{2}, q_{2}\right)\right\|_{\mathbf{V} \times L^{2} \times L^{2}}^{2}(t) \leq c \mathrm{e}^{c_{0} t_{1}}\left\|\left(\boldsymbol{v}_{1}^{0}, T_{1}^{0}, q_{1}^{0}\right)-\left(\boldsymbol{v}_{2}^{0}, T_{2}^{0}, q_{2}^{0}\right)\right\|_{\mathbf{V} \times L^{2} \times L^{2}}^{2} .
$$

In particular, there exists a unique strong solution to (5.5)-(5.7).

Remark 7.2. The replacement of $F$ with its positive part $F^{+}$plays here an essential role. It is linked with positivity and $L^{\infty}$ bounds on the temperature, which will be discussed in 
the subsequent Section 8. Note that Theorem 7.1 applies as well when we keep $F$ instead of replacing it by $F^{+}$, as long as the temperature remains positive and below the bound

$$
T \leq \frac{L R}{c_{p} R_{v}} \simeq 1548 K
$$

which is far higher than any temperature on earth.

7.1. The moist static energy. In order to prove the uniqueness of solutions in Theorem 7.1, we introduce as in [5] the so-called moist static energy function

$$
e=c_{p} T+L q
$$

which is easily seen to satisfy the equation

$$
\frac{\partial e}{\partial t}+\boldsymbol{v} \cdot \nabla e+\omega \frac{\partial e}{\partial p}+\mathcal{A}_{T} e=L\left(\mathcal{A}_{T}-\mathcal{A}_{q}\right) q+\frac{R}{c_{p} p} \omega(e-L q)
$$

along with the boundary conditions

$$
\begin{aligned}
& \text { on } \Gamma_{i}: \frac{\partial e}{\partial p}=-\frac{\alpha_{T}}{\nu_{T}} e+L\left(\frac{\alpha_{T}}{\nu_{T}}-\frac{\alpha_{q}}{\nu_{q}}\right) q, \\
& \text { on } \Gamma_{u}: \frac{\partial e}{\partial p}=0, \\
& \text { on } \Gamma_{\ell}: \frac{\partial e}{\partial \boldsymbol{n}}=0 .
\end{aligned}
$$

Also, the initial condition now reads

$$
e(x, y, p, 0)=e_{0}(x, y, p)
$$

where $e_{0}=c_{p} T_{0}+L q_{0}$. It is clear that proving uniqueness of a quasi-strong solution $(\boldsymbol{v}, T, q)$ is equivalent to showing uniqueness of a quasi-strong solution $(\boldsymbol{v}, e, q)$. Although consideration of the $(\boldsymbol{v}, e, q)$-system introduces some coupling in the linear part of (7.1) and in the boundary conditions on $\Gamma_{i}$, these terms can be handled and we gain the advantage of eliminating the non-Lipschitz term induced by the multivalued function $H\left(q-q_{s}\right)$.

7.2. Proof of Theorem 7.1. In what follows, the letter $c$ will refer to a generic positive constant, which may be calculated in terms of the physical parameters of the problem. Also, $\delta \in(0,1)$ will be a sufficiently small fixed constant. Let $\left(\boldsymbol{v}_{1}, e_{1}, q_{1}\right)$ and $\left(\boldsymbol{v}_{2}, e_{2}, q_{2}\right)$ be two global quasi-strong solutions with initial data $\left(\boldsymbol{v}_{1}^{0}, e_{1}^{0}, q_{1}^{0}\right)$ and $\left(\boldsymbol{v}_{2}^{0}, e_{2}^{0}, q_{2}^{0}\right)$, respectively. The differences

$$
\hat{\boldsymbol{v}}=\boldsymbol{v}_{1}-\boldsymbol{v}_{2}, \quad \hat{e}=e_{1}-e_{2}, \quad \hat{q}=q_{1}-q_{2},
$$


satisfy

$$
\begin{aligned}
\partial_{t} \hat{\boldsymbol{v}}+ & \left(\boldsymbol{v}_{1} \cdot \nabla\right) \hat{\boldsymbol{v}}+(\hat{\boldsymbol{v}} \cdot \nabla) \boldsymbol{v}_{2}+\omega_{1} \partial_{p} \hat{\boldsymbol{v}}+\hat{\omega} \partial_{p} \boldsymbol{v}_{2}+f \boldsymbol{k} \times \hat{\boldsymbol{v}}+\nabla \widehat{\Phi}_{s} \\
& +\nabla \int_{p}^{p_{1}} \frac{R}{c_{p} p^{\prime}}[\hat{e}-L \hat{q}] \mathrm{d} p^{\prime}+\mathcal{A}_{\boldsymbol{v}} \hat{\boldsymbol{v}}=0 \\
\partial_{t} \hat{e}+ & \boldsymbol{v}_{1} \cdot \nabla \hat{e}+\hat{\boldsymbol{v}} \cdot \nabla e_{2}+\omega_{1} \partial_{p} \hat{e}+\hat{\omega} \partial_{p} e_{2}+\mathcal{A}_{T} \hat{e}=L\left(\mathcal{A}_{T}-\mathcal{A}_{q}\right) \hat{q} \\
& +\frac{R}{c_{p} p} \omega_{1}(\hat{e}-L \hat{q})+\frac{R}{c_{p} p} \hat{\omega}\left(e_{2}-L q_{2}\right), \\
\partial_{t} \hat{q}+ & \boldsymbol{v}_{1} \cdot \nabla \hat{q}+\hat{\boldsymbol{v}} \cdot \nabla q_{2}+\omega_{1} \partial_{p} \hat{q}+\hat{\omega} \partial_{p} q_{2}+\mathcal{A}_{q} \hat{q}+\frac{1}{p} \omega_{1}^{-} F^{+}\left(T_{2}\right)\left(h_{q_{1}}-h_{q_{2}}\right) \\
& =-\frac{1}{p} \omega_{1}^{-} h_{q_{1}}\left(F^{+}\left(T_{1}\right)-F^{+}\left(T_{2}\right)\right)-\frac{1}{p} h_{q_{2}} F^{+}\left(T_{2}\right)\left(\omega_{1}^{-}-\omega_{2}^{-}\right) .
\end{aligned}
$$

We now proceed to test each equation in $L^{2}$ by $A_{\boldsymbol{v}} \hat{\boldsymbol{v}}, \hat{e}$ and $\hat{q}$, respectively, taking into account the weak formulation of the equations given by (5.5)-(5.7), appropriately reformulated for $e$ instead of $T$. For $\hat{\boldsymbol{v}}$, we obtain the energy equation

$$
\begin{aligned}
\frac{1}{2} \frac{\mathrm{d}}{\mathrm{d} t}\left|A_{\boldsymbol{v}}^{1 / 2} \hat{\boldsymbol{v}}\right|^{2}+\left|A_{\boldsymbol{v}} \hat{\boldsymbol{v}}\right|^{2}= & -\left(\nabla \int_{p}^{p_{1}} \frac{R}{c_{p} p^{\prime}}[\hat{e}-L \hat{q}] \mathrm{d} p^{\prime}, A_{\boldsymbol{v}} \hat{\boldsymbol{v}}\right)-b\left(\hat{\boldsymbol{v}}, \boldsymbol{v}_{2}, A_{\boldsymbol{v}} \hat{\boldsymbol{v}}\right) \\
& -b\left(\boldsymbol{v}_{1}, \hat{\boldsymbol{v}}, A_{\boldsymbol{v}} \hat{\boldsymbol{v}}\right)-\left(f \boldsymbol{k} \times \hat{\boldsymbol{v}}, A_{\boldsymbol{v}} \hat{\boldsymbol{v}}\right) .
\end{aligned}
$$

We estimate the right-hand side term by term. For the last term, we use the Poincaré inequality for the estimate

$$
\left|\left(f \boldsymbol{k} \times \hat{\boldsymbol{v}}, A_{\boldsymbol{v}} \hat{\boldsymbol{v}}\right)\right| \leq c\|\hat{\boldsymbol{v}}\|^{2}+\frac{1}{24}\left|A_{\boldsymbol{v}} \hat{\boldsymbol{v}}\right|^{2} .
$$

For the first one, as in (3.24) we obtain

$$
\left|\left(\nabla \int_{p}^{p_{1}} \frac{R}{c_{p} p^{\prime}}[\hat{e}-L \hat{q}] \mathrm{d} p^{\prime}, A_{\boldsymbol{v}} \hat{\boldsymbol{v}}\right)\right| \leq c(\|\hat{e}\|+\|\hat{q}\|)\left|A_{\boldsymbol{v}} \hat{\boldsymbol{v}}\right| \leq \frac{1}{24}\left|A_{\boldsymbol{v}} \hat{\boldsymbol{v}}\right|^{2}+\frac{\kappa_{T}}{4 \delta}\|\hat{e}\|^{2}+\frac{\kappa_{q}}{4 \delta^{2}}\|\hat{q}\|^{2} .
$$

Now, the trilinear terms are more difficult and require some care. Note that

$$
b\left(\hat{\boldsymbol{v}}, \boldsymbol{v}_{2}, A_{\boldsymbol{v}} \hat{\boldsymbol{v}}\right)=\left((\hat{\boldsymbol{v}} \cdot \nabla) \boldsymbol{v}_{2}, A_{\boldsymbol{v}} \hat{\boldsymbol{v}}\right)+\left(\hat{\omega} \partial_{p} \boldsymbol{v}_{2}, A_{\boldsymbol{v}} \hat{\boldsymbol{v}}\right),
$$

so that the first part can be estimated in a fairly classical way as

$\left|\left((\hat{\boldsymbol{v}} \cdot \nabla) \boldsymbol{v}_{2}, A_{\boldsymbol{v}} \hat{\boldsymbol{v}}\right)\right| \leq\|\hat{\boldsymbol{v}}\|_{L^{6}}\left\|\nabla \boldsymbol{v}_{2}\right\|_{L^{3}}\left\|\left.A_{\boldsymbol{v}} \hat{\boldsymbol{v}}\left|\leq c\|\hat{\boldsymbol{v}}\|\left\|\boldsymbol{v}_{2}\right\|_{H^{2}}\right| A_{\boldsymbol{v}} \hat{\boldsymbol{v}}\left|\leq \frac{1}{24}\right| A_{\boldsymbol{v}} \hat{\boldsymbol{v}}\right|^{2}+c\right\| \boldsymbol{v}_{2}\left\|_{H^{2}}^{2}\right\| \hat{\boldsymbol{v}} \|^{2}$.

For the second part, we use an anisotropic estimate. We obtain

$$
\left|\left(\hat{\omega} \partial_{p} \boldsymbol{v}_{2}, A_{\boldsymbol{v}} \hat{\boldsymbol{v}}\right)\right| \leq c \int_{\mathcal{M}^{\prime}}\|\hat{\omega}\|_{L_{p}^{\infty}}\left\|\boldsymbol{v}_{2}\right\|_{L_{p}^{2}}\|\| A_{\boldsymbol{v}} \hat{\boldsymbol{v}} \|_{L_{p}^{2}} \mathrm{~d} \mathcal{M}^{\prime}
$$

where the subscript $p$ in the above norms indicates that we have only integrated in the $p$-direction. From the definition of $\hat{\omega}$, we have that

$$
\|\hat{\omega}\|_{L_{p}^{\infty}} \leq c\|\nabla \cdot \hat{\boldsymbol{v}}\|_{L_{p}^{2}}
$$


so that a further use of the Hölder inequality in the $x, y$ direction entails

$$
\begin{aligned}
\left|\left(\hat{\omega} \partial_{p} \boldsymbol{v}_{2}, A_{\boldsymbol{v}} \hat{\boldsymbol{v}}\right)\right| & \leq c\|\hat{\omega}\|_{L_{x, y}^{4} L_{p}^{\infty}}\left\|\partial_{p} \boldsymbol{v}_{2}\right\|_{L_{x, y}^{4} L_{p}^{2}}\|\| A_{\boldsymbol{v}} \hat{\boldsymbol{v}} \|_{L_{x, y}^{2} L_{p}^{2}} \\
& \leq c\|\nabla \cdot \hat{\boldsymbol{v}}\|_{L_{x, y}^{4} L_{p}^{2}}\left\|\partial_{p} \boldsymbol{v}_{2}\right\|_{L_{x, y}^{4} L_{p}^{2}}\|\| A_{\boldsymbol{v}} \hat{\boldsymbol{v}} \|_{L_{x, y}^{2} L_{p}^{2}} \\
& \leq c\|\hat{\boldsymbol{v}}\|^{1 / 2}\left\|\boldsymbol{v}_{2}\right\|^{1 / 2}\left\|\boldsymbol{v}_{2}\right\|_{H^{2}}^{1 / 2}\left|A_{\boldsymbol{v}} \hat{\boldsymbol{v}}\right|^{3 / 2} \\
& \leq \frac{1}{24}\left|A_{\boldsymbol{v}} \hat{\boldsymbol{v}}\right|^{2}+c\left\|\boldsymbol{v}_{2}\right\|^{2}\left\|\boldsymbol{v}_{2}\right\|_{H^{2}}^{2}\|\hat{\boldsymbol{v}}\|^{2}
\end{aligned}
$$

where we used that for $\varphi \in H^{1}$ there holds

$$
\|\varphi\|_{L_{x, y}^{4} L_{p}^{2}} \leq c\|\varphi\|_{L^{2}}^{1 / 2}\|\varphi\|_{H^{1}}^{1 / 2}
$$

In conclusion, we find that

$$
\left|b\left(\hat{\boldsymbol{v}}, \boldsymbol{v}_{2}, A_{\boldsymbol{v}} \hat{\boldsymbol{v}}\right)\right| \leq \frac{1}{12}\left|A_{\boldsymbol{v}} \hat{\boldsymbol{v}}\right|^{2}+c\left(1+\left\|\boldsymbol{v}_{2}\right\|^{2}\right)\left\|\boldsymbol{v}_{2}\right\|_{H^{2}}^{2}\|\hat{\boldsymbol{v}}\|^{2} .
$$

We argue similarly for the third term. Since

$$
b\left(\boldsymbol{v}_{1}, \hat{\boldsymbol{v}}, A_{\boldsymbol{v}} \hat{\boldsymbol{v}}\right)=\left(\left(\boldsymbol{v}_{1} \cdot \nabla\right) \hat{\boldsymbol{v}}, A_{\boldsymbol{v}} \hat{\boldsymbol{v}}\right)+\left(\omega_{1} \partial_{p} \hat{\boldsymbol{v}}, A_{\boldsymbol{v}} \hat{\boldsymbol{v}}\right)
$$

we deduce that

$\left|\left(\left(\boldsymbol{v}_{1} \cdot \nabla\right) \hat{\boldsymbol{v}}, A_{\boldsymbol{v}} \hat{\boldsymbol{v}}\right)\right| \leq\left\|\boldsymbol{v}_{1}\right\|_{L^{6}}\|\nabla \hat{\boldsymbol{v}}\|_{L^{3}}\left|A_{\boldsymbol{v}} \hat{\boldsymbol{v}}\right| \leq c\left\|\boldsymbol{v}_{1}\right\|\|\hat{\boldsymbol{v}}\|^{1 / 2}\left|A_{\boldsymbol{v}} \hat{\boldsymbol{v}}\right|^{3 / 2} \leq \frac{1}{24}\left|A_{\boldsymbol{v}} \hat{\boldsymbol{v}}\right|^{2}+c\left\|\boldsymbol{v}_{1}\right\|^{4}\|\hat{\boldsymbol{v}}\|^{2}$, and

$$
\begin{aligned}
\left|\left(\omega_{1} \partial_{p} \hat{\boldsymbol{v}}, A_{\boldsymbol{v}} \hat{\boldsymbol{v}}\right)\right| & \leq c\left\|\omega_{1}\right\|_{L_{x, y}^{4} L_{p}^{\infty}}\left\|\partial_{p} \hat{\boldsymbol{v}}\right\|_{L_{x, y}^{4} L_{p}^{2}}\left|A_{\boldsymbol{v}} \hat{\boldsymbol{v}}\right| \\
& \leq c\left\|\nabla \cdot \boldsymbol{v}_{1}\right\|_{L_{x, y}^{4} L_{p}^{2}}\left\|\partial_{p} \hat{\boldsymbol{v}}\right\|_{L_{x, y}^{4} L_{p}^{2}}\left|A_{\boldsymbol{v}} \hat{\boldsymbol{v}}\right| \\
& \leq c\left\|\boldsymbol{v}_{1}\right\|^{1 / 2}\left\|\boldsymbol{v}_{1}\right\|_{H^{2}}^{1 / 2}\|\hat{\boldsymbol{v}}\|^{1 / 2}\left|A_{\boldsymbol{v}} \hat{\boldsymbol{v}}\right|^{3 / 2} \\
& \leq \frac{1}{24}\left|A_{\boldsymbol{v}} \hat{\boldsymbol{v}}\right|^{2}+c\left\|\boldsymbol{v}_{1}\right\|^{2}\left\|\boldsymbol{v}_{1}\right\|_{H^{2}}^{2}\|\hat{\boldsymbol{v}}\|^{2}
\end{aligned}
$$

implying

$$
\left|b\left(\boldsymbol{v}_{1}, \hat{\boldsymbol{v}}, A_{\boldsymbol{v}} \hat{\boldsymbol{v}}\right)\right| \leq \frac{1}{12}\left|A_{\boldsymbol{v}} \hat{\boldsymbol{v}}\right|^{2}+c\left(\left\|\boldsymbol{v}_{1}\right\|^{2}+\left\|\boldsymbol{v}_{1}\right\|_{H^{2}}^{2}\right)\left\|\boldsymbol{v}_{1}\right\|^{2}\|\hat{\boldsymbol{v}}\|^{2}
$$

Hence, in light of (7.5)-(7.8), we derive from (7.4) the differential inequality

$$
\begin{aligned}
\frac{\mathrm{d}}{\mathrm{d} t}\|\hat{\boldsymbol{v}}\|^{2}+\left|A_{\boldsymbol{v}} \hat{\boldsymbol{v}}\right|^{2} \leq & \frac{\kappa_{T}}{2 \delta}\|\hat{e}\|^{2}+\frac{\kappa_{q}}{2 \delta^{2}}\|\hat{q}\|^{2} \\
& +c\left[\left\|\boldsymbol{v}_{1}\right\|^{4}+\left\|\boldsymbol{v}_{1}\right\|^{2}\left\|\boldsymbol{v}_{1}\right\|_{H^{2}}^{2}+\left\|\boldsymbol{v}_{2}\right\|_{H^{2}}^{2}+\left\|\boldsymbol{v}_{2}\right\|^{2}\left\|\boldsymbol{v}_{2}\right\|_{H^{2}}^{2}\right]\|\hat{\boldsymbol{v}}\|^{2} .
\end{aligned}
$$

We now turn our attention to the moist static energy equation. Applying the same technique, we have

$$
\begin{aligned}
\frac{1}{2} \frac{\mathrm{d}}{\mathrm{d} t}|\hat{e}|^{2}+a_{T}(\hat{e}, \hat{e})= & -b\left(\hat{\boldsymbol{v}}, e_{2}, \hat{e}\right)+\frac{R}{c_{p}}\left(\frac{1}{p} \omega_{1}(\hat{e}-L \hat{q}), \hat{e}\right) \\
& +\frac{R}{c_{p}}\left(\frac{1}{p} \hat{\omega}\left(e_{2}-L q_{2}\right), \hat{e}\right)+L\left(a_{T}(\hat{q}, \hat{e})-a_{q}(\hat{q}, \hat{e})\right) .
\end{aligned}
$$

Thanks to the orthogonality property of the trilinear form, we have

$$
-b\left(\hat{\boldsymbol{v}}, e_{2}, \hat{e}\right)=b\left(\hat{\boldsymbol{v}}, \hat{e}, e_{2}\right)=\left(\hat{\boldsymbol{v}} \cdot \nabla \hat{e}, e_{2}\right)+\left(\hat{\omega} \partial_{p} \hat{e}, e_{2}\right) .
$$


Therefore, as above,

$$
\left|\left(\hat{\boldsymbol{v}} \cdot \nabla \hat{e}, e_{2}\right)\right| \leq c\|\hat{\boldsymbol{v}}\|_{L^{6}}\|\hat{e}\|\left\|e_{2}\right\|_{L^{3}} \leq c\|\hat{\boldsymbol{v}}\|\|\hat{e}\|\left\|e_{2}\right\| \leq \frac{\kappa_{T}}{10}\|\hat{e}\|^{2}+c\left\|e_{2}\right\|^{2}\|\hat{\boldsymbol{v}}\|^{2},
$$

and by anisotropic estimates,

$$
\begin{aligned}
\left|\left(\hat{\omega} \partial_{p} \hat{e}, e_{2}\right)\right| & \leq c\|\hat{\omega}\|_{L_{x, y}^{4} L_{p}^{\infty}}\left\|\partial_{p} \hat{e}\right\|_{L_{x, y}^{2} L_{p}^{2}}\left\|e_{2}\right\|_{L_{x, y}^{4} L_{p}^{2}} \\
& \leq c\|\nabla \cdot \hat{\boldsymbol{v}}\|_{L_{x, y}^{4} L_{p}^{2}}\left\|\partial_{p} \hat{e}\right\|_{L_{x, y}^{2} L_{p}^{2}}\left\|e_{2}\right\|_{L_{x, y}^{4} L_{p}^{2}} \\
& \leq c\|\nabla \cdot \hat{\boldsymbol{v}}\|_{L_{x, y}^{4} L_{p}^{2}}\left|\partial_{p} \hat{e}\right|\left\|e_{2}\right\|_{L_{x, y}^{4} L_{p}^{2}} \\
& \leq c\|\hat{\boldsymbol{v}}\|^{1 / 2}\left|A_{\boldsymbol{v}} \hat{\boldsymbol{v}}\right|^{1 / 2}\left|e_{2}\right|^{1 / 2}\left\|e_{2}\right\|^{1 / 2}\|\hat{e}\| \\
& \leq \frac{\kappa_{T}}{10}\|\hat{e}\|^{2}+\frac{\delta}{8}\left|A_{\boldsymbol{v}} \hat{\boldsymbol{v}}\right|^{2}+c\left|e_{2}\right|^{2}\left\|e_{2}\right\|^{2}\|\hat{\boldsymbol{v}}\|^{2}
\end{aligned}
$$

Thanks to the continuity of the bilinear forms $a_{T}$ and $a_{q}$, we are able to estimate the last term as

$$
\left|L\left(a_{T}(\hat{q}, \hat{e})-a_{q}(\hat{q}, \hat{e})\right)\right| \leq c\|\hat{e}\|\|\hat{q}\| \leq \frac{\kappa_{T}}{10}\|\hat{e}\|^{2}+\frac{\kappa_{q}}{8 \delta}\|\hat{q}\|^{2} .
$$

Regarding the intermediate terms, we have

$$
\begin{aligned}
\left|\frac{R}{c_{p}}\left(\frac{1}{p} \omega_{1}(\hat{e}-L \hat{q}), \hat{e}\right)\right| & \leq c\left|\omega_{1}\right|\left(\|\hat{e}\|_{L^{4}}^{2}+\|\hat{e}\|_{L^{4}}\|\hat{q}\|_{L^{4}}\right) \\
& \leq c\left\|\boldsymbol{v}_{1}\right\|\left(|\hat{e}|^{1 / 2}\|\hat{e}\|^{3 / 2}+|\hat{e}|^{1 / 4}\|\hat{e}\|^{3 / 4}|\hat{q}|^{1 / 4}\|\hat{q}\|^{3 / 4}\right) \\
& \leq \frac{\kappa_{T}}{10}\|\hat{e}\|^{2}+c\left\|\boldsymbol{v}_{1}\right\|^{4}\left(|\hat{e}|^{2}+|\hat{q}|^{2}\right)+\frac{\kappa_{q}}{8 \delta}\|\hat{q}\|^{2},
\end{aligned}
$$

where we took advantage of the Sobolev embedding $H^{3 / 4} \subset L^{4}$ and interpolation inequalities. In a similar manner,

$$
\begin{aligned}
\left|\frac{R}{c_{p}}\left(\frac{1}{p} \hat{\omega}\left(e_{2}-L q_{2}\right), \hat{e}\right)\right| & \leq c\|\hat{\omega}\|_{L^{6}}\left(\left|e_{2}\right|+\mid q_{2} \|\right)\|\hat{e}\|_{L^{3}} \\
& \leq c\left|A_{\boldsymbol{v}} \hat{\boldsymbol{v}}\right|\left(\left|e_{2}\right|+\left|q_{2}\right|\right)|\hat{e}|^{1 / 2}\|\hat{e}\|^{1 / 2} \\
& \leq \frac{\delta}{8}\left|A_{\boldsymbol{v}} \hat{\boldsymbol{v}}\right|^{2}+\frac{\kappa_{T}}{10}\|\hat{e}\|^{2}+c\left(\left|e_{2}\right|^{4}+\left|q_{2}\right|^{4}\right)|\hat{e}|^{2} .
\end{aligned}
$$

Therefore, in view of (7.11)-(7.15), the energy equation (7.10) becomes

$$
\frac{\mathrm{d}}{\mathrm{d} t}|\hat{e}|^{2}+\kappa_{T}\|\hat{e}\|^{2} \leq \frac{\delta}{2}\left|A_{\boldsymbol{v}} \hat{\boldsymbol{v}}\right|^{2}+\frac{\kappa_{q}}{2 \delta}\|\hat{q}\|^{2}+c\left[1+\left|e_{2}\right|^{2}\right]\left\|e_{2}\right\|^{2}\|\hat{\boldsymbol{v}}\|^{2}+c\left\|\boldsymbol{v}_{1}\right\|^{4}\left(|\hat{e}|^{2}+|\hat{q}|^{2}\right) .
$$

It remains to deal with the specific humidity equation. The corresponding energy equality reads

$$
\begin{aligned}
\frac{1}{2} \frac{\mathrm{d}}{\mathrm{d} t}|\hat{q}|^{2}+a_{q}(\hat{q}, \hat{q})+\left(\frac{1}{p} \omega_{1}^{-} F^{+}\left(T_{2}\right)\left(h_{q_{1}}-h_{q_{2}}\right), \hat{q}\right)=-b\left(\hat{\boldsymbol{v}}, q_{2}, \hat{q}\right) \\
-\left(\frac{1}{p} \omega_{1}^{-} h_{q_{1}}\left(F^{+}\left(T_{1}\right)-F^{+}\left(T_{2}\right)\right), \hat{q}\right)-\left(\frac{1}{p} h_{q_{2}} F^{+}\left(T_{2}\right)\left(\omega_{1}^{-}-\omega_{2}^{-}\right), \hat{q}\right) .
\end{aligned}
$$

As a consequence of the monotonicity of the multivalued map $q \mapsto H\left(q-q_{s}\right)$, we find

$$
\left(\frac{1}{p} \omega_{1}^{-} F^{+}\left(T_{2}\right)\left(h_{q_{1}}-h_{q_{2}}\right), \hat{q}\right) \geq 0
$$


Also, arguing as in (7.11)-(7.12), we infer that

$$
\left|b\left(\hat{\boldsymbol{v}}, q_{2}, \hat{q}\right)\right| \leq \frac{\delta^{2}}{4}\left|A_{\boldsymbol{v}} \hat{\boldsymbol{v}}\right|^{2}+\frac{\kappa_{q}}{6}\|\hat{q}\|^{2}+c\left(1+\left|q_{2}\right|^{2}\right)\left\|q_{2}\right\|^{2}\|\hat{\boldsymbol{v}}\|^{2} .
$$

Moreover, since $\left\|h_{q_{1}}\right\|_{L^{\infty}} \leq 1$ and $F^{+}$is globally Lipschitz-continuous, we can write

$$
\begin{aligned}
\left|\left(\frac{1}{p} \omega_{1}^{-} h_{q_{1}}\left(F^{+}\left(T_{1}\right)-F^{+}\left(T_{2}\right)\right), \hat{q}\right)\right| & \leq c\left|\omega_{1}^{-}\right|\left\|F\left(T_{1}\right)-F\left(T_{2}\right)\right\|_{L^{6}}\|\hat{q}\|_{L^{3}} \\
& \leq c\left\|\boldsymbol{v}_{1}\right\|\|\widehat{T}\|_{L^{6}}|\hat{q}|^{1 / 2}\|\hat{q}\|^{1 / 2} \\
& \leq c\left\|\boldsymbol{v}_{1}\right\|(\|\hat{e}\|+\|\hat{q}\|)|\hat{q}|^{1 / 2}\|\hat{q}\|^{1 / 2} \\
& \leq \frac{\delta \kappa_{T}}{4}\|\hat{e}\|^{2}+\frac{\kappa_{q}}{6}\|\hat{q}\|^{2}+c\left\|\boldsymbol{v}_{1}\right\|^{4}|\hat{q}|^{2} .
\end{aligned}
$$

In a simpler way, recalling that $F$ is a bounded function, we obtain

$$
\left|\left(\frac{1}{p} h_{q_{2}} F^{+}\left(T_{2}\right)\left(\omega_{1}^{-}-\omega_{2}^{-}\right), \hat{q}\right)\right| \leq c\left|\hat{\omega}\|\hat{q} \mid \leq c\| \hat{\boldsymbol{v}}\left\|^{2}+\frac{\kappa_{q}}{6}\right\| \hat{q} \|^{2}\right.
$$

Collecting (7.18)-(7.21), the equation (7.17) turns into

$$
\frac{\mathrm{d}}{\mathrm{d} t}|\hat{q}|^{2}+\kappa_{q}\|\hat{q}\|^{2} \leq \frac{\delta^{2}}{2}\left|A_{\boldsymbol{v}} \hat{\boldsymbol{v}}\right|^{2}+\frac{\delta \kappa_{T}}{2}\|\hat{e}\|^{2}+c\left\|\boldsymbol{v}_{1}\right\|^{4}|\hat{q}|^{2}+c\left(1+\left\|q_{2}\right\|^{2}+\left|q_{2}\right|^{2}\left\|q_{2}\right\|^{2}\right)\|\hat{\boldsymbol{v}}\|^{2}
$$

We now use the fact that the norm $\left|A_{\boldsymbol{v}}^{1 / 2} \hat{\boldsymbol{v}}\right|$ is equivalent to the norm $\|\hat{\boldsymbol{v}}\|$ and put together (7.9), (7.16), and (7.22) in the following way. The energy functional

$$
\Psi(t)=\delta^{2}\left|A_{\boldsymbol{v}}^{1 / 2} \hat{\boldsymbol{v}}(t)\right|^{2}+\delta|\hat{e}(t)|^{2}+|\hat{q}(t)|^{2}
$$

satisfies the differential inequality

$$
\Psi^{\prime}(t) \leq g(t) \Psi(t), \quad t \in\left(0, t_{1}\right]
$$

where

$$
\begin{aligned}
g(t)=c[1 & +\left\|\boldsymbol{v}_{2}\right\|_{H^{2}}^{2}+\left\|\boldsymbol{v}_{1}\right\|^{2}\left\|\boldsymbol{v}_{1}\right\|_{H^{2}}^{2}+\left\|\boldsymbol{v}_{2}\right\|^{2}\left\|\boldsymbol{v}_{2}\right\|_{H^{2}}^{2} \\
& \left.+\left\|\boldsymbol{v}_{1}\right\|^{4}+\left\|e_{2}\right\|^{2}+\left|e_{2}\right|^{2}\left\|e_{2}\right\|^{2}+\left\|q_{2}\right\|^{2}+\left|q_{2}\right|^{2}\left\|q_{2}\right\|^{2}\right] .
\end{aligned}
$$

The fact that $\left(\boldsymbol{v}_{1}, e_{1}, q_{1}\right)$ and $\left(\boldsymbol{v}_{2}, e_{2}, q_{2}\right)$ are quasi-strong solutions ensures that $g \in$ $L^{1}\left(0, t_{1}\right)$. We can therefore apply the standard Gronwall inequality, to obtain the continuous dependence estimate

$$
\Psi(t) \leq \Psi(0) \exp \left(\int_{0}^{t_{1}} g(t) \mathrm{d} t\right)
$$

which is stated explicitly in the statement of Theorem 7.1, and whose proof is now achieved.

Remark 7.3. The only step in which the replacement of $F$ with its positive part $F^{+}$has been used is in (7.18), to exploit monotonicity properties of the set-valued map $H\left(q-q_{s}\right)$. This appears unavoidable at the moment, and it allows to circumvent the difficulty of dealing with non-Lipschitz nonlinearities, by using instead monotonicity arguments. 


\section{MAXIMUM PRINCIPLES}

In order to prove uniqueness of solutions, we replaced the nonlinear function $F$ by $F^{+}$. This amounts to requiring that the temperature distribution $T$ satisfies the bounds given by (2.22), namely

$$
0 \leq T \leq \frac{L R}{c_{p} R_{v}} \simeq 1548 K, \quad \text { a.e. in } \mathcal{M} \times\left[0, t_{1}\right] .
$$

Note in addition that the physical model (2.1)-(2.6) is probably not valid anymore if $T>1548 K$, for possibile utilization in extraterrestrial atmospheres.

In [5], physical bounds of the form (8.1) were derived for both the temperature and the specific humidity. Here, the picture is quite similar, despite the fact that the velocity vector field $\boldsymbol{v}$ is not a given datum anymore, but part of the unknowns. In particular, one can repeat word for word the arguments in [5, Proposition 4.1] to obtain a positivity result.

Proposition 8.1. With the hypotheses in Theorem 6.3, suppose $T_{0}, q_{0}$ and $S_{T}, S_{q}$ are positive functions. Then we have $T(\cdot, t), q(\cdot, t) \geq 0$ almost everywhere in $\mathcal{M}$ and for every $t \in\left[0, t_{1}\right]$.

The main difference compared to [5] concerns the upper bounds. Specifically, a key assumption there was that $\omega \in L^{\infty}\left(\mathcal{M} \times\left(0, t_{1}\right)\right)$, which is not compatible with our estimates above on strong solutions. Here, we will instead exploit a technique reminiscent of the famous nonlinear iteration of De Giorgi [7] in order to prove the following $L^{\infty}$ bound on the temperature.

Proposition 8.2. With the hypotheses in Theorem 6.3, we suppose that $T_{0} \in L^{\infty}$ is positive and we further assume that $S_{T} \in L^{2}\left(0, t_{1} ; H^{1}\right)$. Then there exists a positive constant $M_{1}$ such that

$$
0 \leq T \leq M_{1}, \quad \text { a.e. in } \mathcal{M} \times\left[0, t_{1}\right]
$$

Specifically, we have

$$
M_{1}=M_{1}\left(t_{1},\left\|\boldsymbol{v}_{0}\right\|,\left\|T_{0}\right\|,\left\|T_{0}\right\|_{L^{\infty}},\left\|q_{0}\right\|,\left\|S_{T}\right\|_{L^{2}\left(0, t_{1} ; H^{1}\right)},\left\|\left(\boldsymbol{S}_{\boldsymbol{v}}, S_{T}, S_{q}\right)\right\|_{L^{2}\left(0, t_{1} ; H\right)}\right) .
$$

The only extra requirement is $S_{T} \in L^{2}\left(0, t_{1} ; H^{1}\right)$. The above proposition will be proven in the subsequent Section 8.1. Moreover, as the equation for $q$ is similar to the equation for $T$, an analogous result holds for the specific humidity.

Proposition 8.3. With the hypotheses in Theorem 6.3, assume that $q_{0} \in L^{\infty}$ is positive and $S_{q} \in L^{2}\left(0, t_{1} ; H^{1}\right)$. Then there exist a positive constant $M_{2}$ such that

$$
0 \leq q \leq M_{2}, \quad \text { a.e. in } \mathcal{M} \times\left[0, t_{1}\right] .
$$

Specifically, we have

$$
M_{2}=M_{2}\left(t_{1},\left\|\boldsymbol{v}_{0}\right\|,\left\|T_{0}\right\|,\left\|q_{0}\right\|,\left\|q_{0}\right\|_{L^{\infty}},\left\|S_{q}\right\|_{L^{2}\left(0, t_{1} ; H^{1}\right)},\left\|\left(\boldsymbol{S}_{\boldsymbol{v}}, S_{T}, S_{q}\right)\right\|_{L^{2}\left(0, t_{1} ; H\right)}\right) .
$$

The proof of the above proposition will be left to the reader, as it is shorter and simpler than that of Proposition 8.2.

Remark 8.4. The main drawback of such bounds is the dependence on the final time $t_{1}$. However, this is not caused by a flaw in the proof, but rather from the non-uniformity of 
the basic $L^{2}$ energy estimate (5.8). This seems to be an intrinsic feature of the model, caused essentially by the presence of the anti-dissipative term

$$
-\frac{R}{c_{p} p} \omega T
$$

in the temperature equation.

The methods of [5] work here as well for the specific humidity, without further assumptions on $\omega$. Complementary to Proposition 8.3 in the (physical) case of zero forcing, we also have the following:

Proposition 8.5. With the hypotheses in Theorem 6.3, assume that $q$ is a strong solution to $(5.7)$ on $\left[0, t_{1}\right]$, with the nonlinear function $F$ replaced by its positive part $F^{+}$, and with positive initial data $q_{0} \in L^{\infty}$. If $S_{q}=0$, then

$$
0 \leq q \leq\left\|q_{0}\right\|_{L^{\infty}} \quad \text { a.e. in } \mathcal{M} \times\left[0, t_{1}\right] .
$$

Remark 8.6. Property (8.4) is what one should expect from the physical considerations. Indeed, the specific humidity is the (dimensionless) ratio of the mass of water vapor to the total mass of the system. One therefore expects that

$$
q \in[0,1], \quad \text { a.e. in } \mathcal{M} \times\left[0, t_{1}\right] .
$$

Moreover, (8.4) also implies that if the initial datum is smaller than the saturation concentration, then the under-saturated regime persists for all times. If, as it should be ([14, p. 163]), $q_{s}$ depends on $T$ (typically, $q_{s}=\frac{C}{p} \mathrm{e}^{-L / R_{v} T}$, as in [14]), then a decrease of $T$ produces a decrease of $q_{s}$, thus increasing the chances of supersaturation. The mathematical theory of the case where $q_{s}$ depends on $T$ has been developed in [3].

Remark 8.7. We observe once more that the truncation of $F$ is not required to prove the maximum principle neither for the temperature nor for the specific humidity, as opposed to what was proved in [5], where the truncation of $F$ was assumed to prove the upper bound for $q$. Indeed, it was there crucial an estimate of the type

$$
-\left\langle\frac{1}{p} \omega^{-} h_{q} F^{+}(T),\left[q-M_{2}\right]^{+}\right\rangle \leq 0
$$

when using the Stampacchia method on the specific humidity equation. However, a bound in space and time (independent of the final time $t_{1}$ ), like in (8.1) seems to be out of reach at the moment: the possibility of exponential growth of the $L^{2}$ norm of the temperature (see (5.8)) caused by the anti-dissipative term mentioned in Remark 8.4 prevents us to prove uniform $L^{\infty}$ bounds as well (see (8.9) and (8.16)).

8.1. Upper bounds for the temperature via De Giorgi iterations. We prove here Proposition 8.2. We consider the weak formulation of the temperature equation (5.6), and take the test function $\widetilde{T}$ to be $T_{\lambda}=[T-\lambda]^{+}$, where $\lambda$ is any positive constant such that

$$
\lambda \geq\left\|T_{0}\right\|_{L^{\infty}} .
$$

The corresponding energy equation reads

$$
\begin{aligned}
\frac{1}{2} \frac{\mathrm{d}}{\mathrm{d} t}\left|T_{\lambda}\right|^{2}+a_{T}\left(T_{\lambda}, T_{\lambda}\right)+\lambda \alpha_{T} \int_{\Gamma_{i}}\left(\frac{g p_{1}}{R \bar{T}}\right)^{2} T_{\lambda} \mathrm{d} \Gamma_{i}= & m_{T}\left(\omega, T_{\lambda}, T_{\lambda}\right)+m_{T}\left(\omega, \lambda, T_{\lambda}\right) \\
& +\frac{L}{c_{p}}\left(D\left(\omega, T, h_{q}\right), T_{\lambda}\right)+\left(S_{T}, T_{\lambda}\right),
\end{aligned}
$$


where we took advantage of the orthogonality property of the trilinear form $b$, namely

$$
b\left(\boldsymbol{v}, T, T_{\lambda}\right)=b\left(\boldsymbol{v}, T-\lambda, T_{\lambda}\right)=b\left(\boldsymbol{v}, T_{\lambda}, T_{\lambda}\right)=0 .
$$

As a consequence, we have

$$
\begin{aligned}
\frac{\mathrm{d}}{\mathrm{d} t}\left|T_{\lambda}\right|^{2}+2 \kappa_{T}\left\|T_{\lambda}\right\|^{2} \leq & c_{1} \int_{\mathcal{M}}|\omega|\left|T_{\lambda}\right|^{2} \mathrm{~d} \mathcal{M}+c_{1} \lambda \int_{\mathcal{M}}|\omega|\left|T_{\lambda}\right| \mathrm{d} \mathcal{M} \\
& +c_{2} \int_{\mathcal{M}}|\omega|\left|T_{\lambda}\right| \mathrm{d} \mathcal{M}+2 \int_{\mathcal{M}}\left|S_{T}\right|\left|T_{\lambda}\right| \mathrm{d} \mathcal{M},
\end{aligned}
$$

where

$$
c_{1}=\frac{R c_{p}}{p_{0}}, \quad c_{2}=\frac{L c_{p}}{p_{0}} C_{F}, \quad\left(C_{F}>0 \text { from }(2.19)\right) .
$$

Hence, integrating on $(0, t)$ and using the fact that $T_{\lambda}(0)=0$, we obtain

$$
\begin{aligned}
\sup _{t \in\left[0, t_{1}\right]}\left|T_{\lambda}(t)\right|^{2}+2 \kappa_{T} \int_{0}^{t_{1}}\left\|T_{\lambda}(t)\right\|^{2} \mathrm{~d} t \leq & c_{1} \int_{0}^{t_{1}} \int_{\mathcal{M}}|\omega|\left|T_{\lambda}\right|^{2} \mathrm{~d} \mathcal{M} \mathrm{d} t+c_{1} \lambda \int_{0}^{t_{1}} \int_{\mathcal{M}}|\omega|\left|T_{\lambda}\right| \mathrm{d} \mathcal{M} \mathrm{d} t \\
& +c_{2} \int_{0}^{t_{1}} \int_{\mathcal{M}}|\omega|\left|T_{\lambda}\right| \mathrm{d} \mathcal{M} \mathrm{d} t+2 \int_{0}^{t_{1}} \int_{\mathcal{M}}\left|S_{T}\right|\left|T_{\lambda}\right| \mathrm{d} \mathcal{M} \mathrm{d} t .
\end{aligned}
$$

For $M \geq 2\left\|T_{0}\right\|_{L^{\infty}}$ to be fixed later, we define the increasing sequence of positive numbers

$$
\lambda_{k}=M\left(1-2^{-k}\right), \quad k \geq 0,
$$

and set $T_{k}=T_{\lambda_{k}}$. Note that $T_{k} \leq T_{k-1}$ for every $k \geq 0$, and, moreover,

$$
T_{k-1} \geq 2^{-k} M \quad \text { on }\left\{(x, y, p, t): T_{k}(x, y, p, t)>0\right\} .
$$

In turn,

$$
\mathbb{1}_{\left\{T_{k}>0\right\}} \leq \frac{2^{k}}{M} T_{k-1}, \quad \forall k \geq 1
$$

Defining

$$
Q_{k}=\sup _{t \in\left[0, t_{1}\right]}\left|T_{k}(t)\right|^{2}+2 \kappa_{T} \int_{0}^{t_{1}}\left\|T_{k}(t)\right\|^{2} \mathrm{~d} t
$$

we infer from (8.5) that, for every $k \geq 1$,

$$
\begin{aligned}
Q_{k} \leq & c_{1} \int_{0}^{t_{1}} \int_{\mathcal{M}}|\omega|\left|T_{k}\right|^{2} \mathrm{~d} \mathcal{M} \mathrm{d} t+c_{1} M \int_{0}^{t_{1}} \int_{\mathcal{M}}|\omega|\left|T_{k}\right| \mathrm{d} \mathcal{M} \mathrm{d} t \\
& +c_{2} \int_{0}^{t_{1}} \int_{\mathcal{M}}|\omega|\left|T_{k}\right| \mathrm{d} \mathcal{M} \mathrm{d} t+2 \int_{0}^{t_{1}} \int_{\mathcal{M}}\left|S_{T}\right|\left|T_{k}\right| \mathrm{d} \mathcal{M} \mathrm{d} t
\end{aligned}
$$

where we used $\left\|T_{0}\right\|_{L^{\infty}} \leq \lambda_{k} \leq M$ for each $k \geq 1$ from our choice of $M$. Moreover, from the estimate (5.8), which can now be extended up to $t_{1}$ in view of the global existence result in Theorem 6.3, we have

$Q_{0}=\sup _{t \in\left[0, t_{1}\right]}|T(t)|^{2}+2 \kappa_{T} \int_{0}^{t_{1}}\|T(t)\|^{2} \mathrm{~d} t \leq C e^{C t_{1}}\left(\left|\left(\boldsymbol{v}_{0}, T_{0}, q_{0}\right)\right|^{2}+\left\|\left(\boldsymbol{S}_{\boldsymbol{v}}, S_{T}, S_{q}\right)\right\|_{L^{2}\left(0, t_{1} ; H\right)}^{2}\right)$. 
We now proceed with estimating the right hand side of (8.8). Note that it is enough to find upper bounds for the first two terms, as the third and fourth terms are very similar to the second one.

We have

$$
\begin{aligned}
\int_{0}^{t_{1}} \int_{\mathcal{M}}\left|\omega \| T_{k}\right|^{2} \mathrm{~d} \mathcal{M} \mathrm{d} t & \leq \int_{0}^{t_{1}}\|\omega\|_{L^{6}}\left(\int_{\mathcal{M}}\left|T_{k}\right|^{12 / 5} \mathrm{~d} \mathcal{M}\right)^{5 / 6} \mathrm{~d} t \\
& \leq\|\omega\|_{L^{2}\left(0, t_{1} ; H^{1}\right)}\left(\int_{0}^{t_{1}}\left(\int_{\mathcal{M}}\left|T_{k}\right|^{12 / 5} \mathrm{~d} \mathcal{M}\right)^{5 / 3} \mathrm{~d} t\right)^{1 / 2} .
\end{aligned}
$$

In view of (8.6) and the fact that $T_{k} \leq T_{k-1}$, we have

$$
\begin{aligned}
\int_{0}^{t_{1}}\left(\int_{\mathcal{M}}\left|T_{k}\right|^{12 / 5} \mathrm{~d} \mathcal{M}\right)^{5 / 3} \mathrm{~d} t & \leq \int_{0}^{t_{1}}\left(\int_{\mathcal{M}}\left|T_{k-1}\right|^{12 / 5} \mathbb{1}_{\left\{T_{k}>0\right\}}^{2 / 5} \mathrm{~d} \mathcal{M}\right)^{5 / 3} \mathrm{~d} t \\
& \leq \frac{2^{2 k / 3}}{M^{2 / 3}} \int_{0}^{t_{1}}\left(\int_{\mathcal{M}}\left|T_{k-1}\right|^{14 / 5} \mathrm{~d} \mathcal{M}\right)^{5 / 3} \mathrm{~d} t \\
& =\frac{2^{2 k / 3}}{M^{2 / 3}} \int_{0}^{t_{1}}\left\|T_{k-1}\right\|_{L^{14 / 5}}^{14 / 3} \mathrm{~d} t
\end{aligned}
$$

Taking advantage of the interpolation inequality

$$
\|\varphi\|_{L^{14 / 5}} \leq\|\varphi\|_{L^{2}}^{4 / 7}\|\varphi\|_{L^{6}}^{3 / 7}
$$

we find that

$$
\begin{aligned}
\int_{0}^{t_{1}}\left(\int_{\mathcal{M}}\left|T_{k}\right|^{12 / 5} \mathrm{~d} \mathcal{M}\right)^{5 / 3} \mathrm{~d} t & \leq \frac{2^{2 k / 3}}{M^{2 / 3}} \int_{0}^{t_{1}}\left\|T_{k-1}\right\|_{L^{2}}^{8 / 3}\left\|T_{k-1}\right\|_{L^{6}}^{2} \mathrm{~d} t \\
& \leq \frac{2^{2 k / 3}}{M^{2 / 3}} \sup _{t \in\left[0, t_{1}\right]}\left|T_{k-1}(t)\right|^{8 / 3} \int_{0}^{t_{1}}\left\|T_{k-1}(t)\right\|^{2} \mathrm{~d} t \\
& \leq \frac{2^{2 k / 3}}{2 \kappa_{T} M^{2 / 3}} Q_{k-1}^{7 / 3}
\end{aligned}
$$

Finally, we obtain the nonlinear estimate

$$
c_{1} \int_{0}^{t_{1}} \int_{\mathcal{M}}|\omega|\left|T_{k}\right|^{2} \mathrm{~d} \mathcal{M} \mathrm{d} t \leq \frac{c_{1}}{\sqrt{2 \kappa_{T}}}\|\boldsymbol{v}\|_{L^{2}\left(0, t_{1} ; H^{2}\right)} \frac{2^{k / 3}}{M^{1 / 3}} Q_{k-1}^{7 / 6}
$$

Now for the second term, we use similar arguments as before and obtain

$$
\int_{0}^{t_{1}} \int_{\mathcal{M}}|\omega|\left|T_{k}\right| \mathrm{d} \mathcal{M} \mathrm{d} t \leq\|\boldsymbol{v}\|_{L^{2}\left(0, t_{1} ; H^{2}\right)}\left[\int_{0}^{t_{1}}\left(\int_{\mathcal{M}}\left|T_{k}\right|^{6 / 5} \mathrm{~d} \mathcal{M}\right)^{5 / 3} \mathrm{~d} t\right]^{1 / 2} .
$$


Using again (8.6), we can estimate the above term as

$$
\begin{aligned}
\int_{0}^{t_{1}}\left(\int_{\mathcal{M}}\left|T_{k}\right|^{6 / 5} \mathrm{~d} \mathcal{M}\right)^{5 / 3} \mathrm{~d} t & \leq \int_{0}^{t_{1}}\left(\int_{\mathcal{M}}\left|T_{k-1}\right|^{6 / 5} \mathbb{1}_{\left\{T_{k}>0\right\}}^{8 / 5} \mathrm{~d} \mathcal{M}\right)^{5 / 3} \mathrm{~d} t \\
& \leq \frac{2^{8 k / 3}}{M^{8 / 3}} \int_{0}^{t_{1}}\left(\int_{\mathcal{M}}\left|T_{k-1}\right|^{14 / 5} \mathrm{~d} \mathcal{M}\right)^{5 / 3} \mathrm{~d} t \\
& =\frac{2^{8 k / 3}}{M^{8 / 3}} \int_{0}^{t_{1}}\left\|T_{k-1}\right\|_{L^{14 / 5}}^{14 / 3} \mathrm{~d} t .
\end{aligned}
$$

Up to the constant in front of the integral, we are in the same situation as above. Hence,

$$
\int_{0}^{t_{1}}\left(\int_{\mathcal{M}}\left|T_{k}\right|^{6 / 5} \mathrm{~d} \mathcal{M}\right)^{5 / 3} \mathrm{~d} t \leq \frac{2^{8 k / 3}}{2 \kappa_{T} M^{8 / 3}} Q_{k-1}^{7 / 3}
$$

and thus

$$
c_{1} M \int_{0}^{t_{1}} \int_{\mathcal{M}}|\omega|\left|T_{k}\right| \mathrm{d} \mathcal{M} \mathrm{d} t \leq \frac{c_{1}}{\sqrt{2 \kappa_{T}}}\|\boldsymbol{v}\|_{L^{2}\left(0, t_{1} ; H^{2}\right)} \frac{2^{4 k / 3}}{M^{1 / 3}} Q_{k-1}^{7 / 6} .
$$

The third and fourth terms are estimated in the exact same way as the second term. We therefore obtain

$$
c_{2} \int_{0}^{t_{1}} \int_{\mathcal{M}}|\omega|\left|T_{k}\right| \mathrm{d} \mathcal{M} \mathrm{d} t \leq \frac{c_{2}}{\sqrt{2 \kappa_{T}}}\|\boldsymbol{v}\|_{L^{2}\left(0, t_{1} ; H^{2}\right)} \frac{2^{4 k / 3}}{M^{4 / 3}} Q_{k-1}^{7 / 6}
$$

and

$$
2 \int_{0}^{t_{1}} \int_{\mathcal{M}}\left|S_{T}\left\|T_{k} \mid \mathrm{d} \mathcal{M} \mathrm{d} t \leq \frac{2}{\sqrt{2 \kappa_{T}}}\right\| S_{T} \|_{L^{2}\left(0, t_{1} ; H^{1}\right)} \frac{2^{4 k / 3}}{M^{4 / 3}} Q_{k-1}^{7 / 6} .\right.
$$

In light of (8.8) and by (8.10)-(8.13), the nonlinear iteration may be written as

$$
Q_{k} \leq \frac{C_{0}}{M^{1 / 3}} 4^{k} Q_{k-1}^{7 / 6}, \quad \forall k \geq 1
$$

where we have assumed $M \geq 1$, and we have set

$$
C_{0}:=C_{0}\left(t_{1},\left\|\boldsymbol{v}_{0}\right\|,\left\|T_{0}\right\|,\left\|q_{0}\right\|,\left\|S_{T}\right\|_{L^{2}\left(0, t_{1} ; H^{1}\right)},\left\|\left(\boldsymbol{S}_{\boldsymbol{v}}, S_{T}, S_{q}\right)\right\|_{L^{2}\left(0, t_{1} ; H\right)}\right)
$$

to be the bound on the velocity field given by Theorem 6.3. Thanks to the nonlinearity in $(8.14)$,

$$
\lim _{k \rightarrow \infty} Q_{k}=0,
$$

provided we choose $M$ sufficiently large. In fact, it is possible to find that $Q_{k}$ satisfies the explicit bound

$$
Q_{k} \leq\left(\frac{Q_{0} 4^{42} C_{0}^{6}}{M^{2}}\right)^{\left(\frac{7}{6}\right)^{k}}\left(\frac{M^{1 / 3}}{4^{7} C_{0}}\right)^{6} 4^{-6 k}, \quad \forall k \geq 1 .
$$

Hence, it suffices to impose the condition

$$
\frac{Q_{0} 4^{42} C_{0}^{6}}{M^{2}} \leq 1,
$$

i.e.,

$$
M \geq \sqrt{Q_{0}} 2^{42} C_{0}^{3} .
$$


In turn, since $Q_{0} \leq C_{0}$, we require that

$$
M \geq 2^{42} C_{0}^{7 / 2}
$$

As $k \rightarrow \infty$, we have that $\lambda_{k} \rightarrow M$, and from (8.7) we learn that

$$
[T-M]^{+}=\lim _{k \rightarrow \infty} T_{k}=0
$$

from which the upper bound on the temperature follows.

\section{Appendix A. Primitive equations of the Atmosphere}

In [20], the authors proved the global existence of strong solutions for the primitive equations of the ocean with Dirichlet boundary conditions for the side boundary. Adapting these techniques, we are able to prove a similar result for the primitive equations of the atmosphere with free-slip boundary conditions for the side boundary. The result in Theorem A.1 below is essential for deriving the time-uniform $H^{1}$-estimate for the velocity $\boldsymbol{v}$ and hence the global existence of strong solutions for the primitive equations with saturation in Section 6.

In this appendix, we decouple the primitive equations of the atmosphere from the temperature and the humidity, and consider the equation

$$
\begin{aligned}
& \frac{\partial \boldsymbol{v}}{\partial t}+\boldsymbol{v} \cdot \nabla \boldsymbol{v}+\omega \frac{\partial \boldsymbol{v}}{\partial p}+\nabla \Phi_{s}+\mathcal{A}_{\boldsymbol{v}} \boldsymbol{v}=\boldsymbol{S}_{\boldsymbol{v}} \\
& \operatorname{div} \boldsymbol{v}+\frac{\partial \omega}{\partial p}=0
\end{aligned}
$$

with initial condition

$$
\boldsymbol{v}(x, y, p, 0)=\boldsymbol{v}_{0}(x, y, p)
$$

and boundary conditions

$$
\left.\left(\nu_{\boldsymbol{v}} \frac{\partial \boldsymbol{v}}{\partial p}+\alpha_{\boldsymbol{v}} \boldsymbol{v}\right)\right|_{\Gamma_{i}}=\left.\omega\right|_{\Gamma_{i}}=0,\left.\quad \frac{\partial \boldsymbol{v}}{\partial p}\right|_{\Gamma_{u}}=\left.\omega\right|_{\Gamma_{u}}=0,\left.\quad(\boldsymbol{v} \cdot \boldsymbol{n})\right|_{\Gamma_{\ell}}=\left.\frac{\partial(\boldsymbol{v} \cdot \boldsymbol{\tau})}{\partial \boldsymbol{n}}\right|_{\Gamma_{\ell}}=0 .
$$

Our result resembles those in [20, Theorem 2.1] and [21, Theorem 2.1]. The proof of Theorem A.1 below actually follows the lines of [20, Theorem 2.1] and [21, Theorem 2.1], where the difference is that instead of the ordinary three-dimensional Laplace operator, we consider the more complicated operator $\mathcal{A}_{\boldsymbol{v}}$ in (A.1). This does not create additional difficulties for the energy estimates below. Nonetheless, we present the full details for the proof of Theorem A.1 below for the sake of completeness.

Theorem A.1. Let the space $\mathbf{V}$ be defined as in Section 3.3 and assume that $\boldsymbol{v}_{0} \in \mathbf{V}$ and $\boldsymbol{S}_{\boldsymbol{v}} \in L^{2}\left(0, t_{1} ; L^{2}\right)$. Then there exists a unique strong solution

$$
\boldsymbol{v} \in L^{\infty}\left(0, t_{1} ; \mathbf{V}\right) \cap L^{2}\left(0, t_{1} ; H^{2}\right)
$$

of the primitive equations (A.1)-(A.3).

Proof. In the following, we will write the norms with respect to the spaces explicitly, that is we write $\|\cdot\|_{\mathbf{V}}$ denoting the norm on the space $\mathbf{V}$ and $|\cdot|$ the absolute value. We also denote by $\|\cdot\|_{L^{p}}\left(\right.$ or $\left.\|\cdot\|_{L^{p}(\mathcal{M})}\right)$ the norm on the space $L^{p}(\mathcal{M})$ and by $\|\cdot\|_{L_{x, y}^{p}}\left(\right.$ or $\left.\|\cdot\|_{L^{p}\left(\mathcal{M}^{\prime}\right)}\right)$ the norm on the space $L^{p}\left(\mathcal{M}^{\prime}\right)$ for $1 \leq p \leq \infty$. 
Following $[20,21]$, we first write $(\mathrm{A} .1)_{1}$ in component form:

$$
\frac{\partial u_{k}}{\partial t}+\sum_{j=1}^{3} \partial_{j}\left(u_{j} u_{k}\right)+\partial_{k} \Phi_{s}+\mathcal{A}_{\boldsymbol{v}} u_{k}=S_{k}, \quad k=1,2,
$$

where

$$
\boldsymbol{u}=\left(u_{1}, u_{2}, u_{3}\right)=(\boldsymbol{v}, \omega)=\left(v_{1}, v_{2}, \omega\right), \quad \boldsymbol{S}_{\boldsymbol{v}}=\left(S_{1}, S_{2}\right) .
$$

Local existence of strong solutions to (A.1)-(A.3) is well-known (see e.g. [29]) and to prove Theorem A.1 it suffices to show the existence of a constant $\widetilde{M}>0$ such that $\|\boldsymbol{v}(\cdot, t)\|_{\mathbf{V}} \leq \widetilde{M}$ for all $t \in\left[0, t_{1}\right]$.

Let $0 \leq \tau_{1}<\tau_{2} \leq t_{1}$. In what follows, all the computations are understood for $t \in\left[\tau_{1}, \tau_{2}\right]$. Before entering into the estimates, we introduce the average operator $M$ in the vertical direction:

$$
M \boldsymbol{v}(x, y)=\frac{1}{h} \int_{p_{0}}^{p_{1}} \boldsymbol{v}(x, y, p) \mathrm{d} p, \quad(x, y) \in \mathcal{M}^{\prime},
$$

where $h:=p_{1}-p_{0}$.

We first obtain an $L^{6}$-estimate on $\boldsymbol{v}$. We multiply (A.4) by $u_{k}^{5}$, where $k=1,2$, integrate over $\mathcal{M}$, and sum the resulting equations. We arrive at

$$
\begin{array}{r}
\frac{1}{6} \sum_{k=1}^{2} \frac{\mathrm{d}}{\mathrm{d} t}\left\|u_{k}\right\|_{L^{6}}^{6}+\frac{5 \mu_{\boldsymbol{v}}}{9} \sum_{k=1}^{2} \int_{\mathcal{M}}\left|\nabla\left(u_{k}^{3}\right)\right|^{2} \mathrm{~d} \mathcal{M}+\frac{5 \nu_{\boldsymbol{v}}}{9} \sum_{k=1}^{2} \int_{\mathcal{M}}\left(\frac{g p}{R \bar{T}}\right)^{2}\left|\partial_{p}\left(u_{k}\right)\right|^{3} \mathrm{~d} \mathcal{M} \\
+\alpha_{\boldsymbol{v}} \sum_{k=1}^{2} \int_{\Gamma_{i}}\left(\frac{g p_{1}}{R \bar{T}}\right)^{2}\left|u_{k}\right|^{6} \mathrm{~d} \Gamma_{i}=-\sum_{k=1}^{2} \int_{\mathcal{M}} u_{k}^{5} \partial_{k} \Phi_{s} \mathrm{~d} \mathcal{M}+\sum_{k=1}^{2} \int_{\mathcal{M}} S_{k} u_{k}^{5} \mathrm{~d} \mathcal{M} \\
=-h \sum_{k=1}^{2} \int_{\mathcal{M}^{\prime}} M\left(u_{k}^{5}\right) \partial_{k} \Phi_{s} \mathrm{~d} \mathcal{M}^{\prime}+\sum_{k=1}^{2} \int_{\mathcal{M}} S_{k} u_{k}^{5} \mathrm{~d} \mathcal{M} .
\end{array}
$$

As in [20], the first term on the right-hand side of (A.5) can be bounded using Hölder's inequality and the Sobolev embedding $W^{1,6 / 5}\left(\mathcal{M}^{\prime}\right) \hookrightarrow L^{3}\left(\mathcal{M}^{\prime}\right)$ by

$$
C \sum_{k=1}^{2}\left\|M\left(u_{k}^{5}\right)\right\|_{L^{3}}\left\|\nabla \Phi_{s}\right\|_{L^{3 / 2}} \leq C \sum_{k=1}^{2}\left(\left\|\nabla M\left(u_{k}^{5}\right)\right\|_{L^{6 / 5}}+\left\|M\left(u_{k}^{5}\right)\right\|_{L^{6 / 5}}\right)\left\|\nabla \Phi_{s}\right\|_{L_{x, y}^{3 / 2}} .
$$

Now, we estimate

$\left\|\nabla M\left(u_{k}^{5}\right)\right\|_{L^{6 / 5}} \leq C\left\|u_{k}^{2} \nabla\left(u_{k}^{3}\right)\right\|_{L^{6 / 5}} \leq C J^{2} \bar{J}^{3}, \quad\left\|M\left(u_{k}^{5}\right)\right\|_{L^{6 / 5}} \leq C\left\|u_{k}\right\|_{L^{6}}^{5} \leq C J^{5} \leq C J^{2} \bar{J}^{3}$, where we denoted

$$
J(t)=\left(\left\|\left(u_{1}^{3}, u_{2}^{3}\right)\right\|_{L^{2}}^{2}\right)^{1 / 6}=\left(\sum_{k=1}^{2}\left\|u_{k}\right\|_{L^{6}}^{6}\right)^{1 / 6}
$$

and

$$
\begin{aligned}
\bar{J}(t)= & \left(\left\|\left(u_{1}^{3}, u_{2}^{3}\right)\right\|_{\mathbf{V}}^{2}\right)^{1 / 6}=\left(\sum_{k=1}^{2} \int_{\mathcal{M}}\left|\nabla\left(u_{k}^{3}\right)\right|^{2} \mathrm{~d} \mathcal{M}\right. \\
& \left.+\sum_{k=1}^{2} \int_{\mathcal{M}}\left(\frac{g p}{R \bar{T}}\right)^{2}\left|\partial_{p}\left(u_{k}^{3}\right)\right|^{2} \mathrm{~d} \mathcal{M}+\sum_{k=1}^{2} \int_{\Gamma_{i}}\left(\frac{g p_{1}}{R \bar{T}}\right)^{2}\left|u_{k}\right|^{6} \mathrm{~d} \Gamma_{i}\right)^{1 / 6},
\end{aligned}
$$


and used the Poincaré's inequality,

$$
J(t) \leq C \bar{J}(t)
$$

The second term on the right side of (A.5) can be estimated by

$$
\sum_{k=1}^{2}\left\|S_{k}\right\|_{L^{2}}\left\|u_{k}^{5}\right\|_{L^{2}}=\sum_{k=1}^{2}\left\|S_{k}\right\|_{L^{2}}\left\|u_{k}^{3}\right\|_{L^{10 / 3}}^{5 / 3} \leq \sum_{k=1}^{2}\left\|S_{k}\right\|_{L^{2}}\left\|u_{k}^{3}\right\|_{L^{2}}^{2 / 3}\left\|u_{k}^{3}\right\|_{L^{2}}
$$

which is further bounded using the Poincaré inequality by

$$
\sum_{k=1}^{2}\left\|S_{k}\right\|_{L^{2}}\left\|u_{k}\right\|_{L^{6}}^{2}\left\|\left(u_{1}^{3}, u_{2}^{3}\right)\right\|_{\mathbf{v}} \leq C \mathbf{S} J^{2} \bar{J}^{3}
$$

where

$$
\mathbf{S}(t)=\left(\sum_{k=1}^{2}\left\|S_{k}(t)\right\|_{L^{2}}^{2}\right)^{1 / 2}
$$

Now, we recall that $\kappa_{\boldsymbol{v}}=\min \left(\mu_{\boldsymbol{v}}, \nu_{\boldsymbol{v}}, \alpha_{\boldsymbol{v}}\right)$ and deduce from (A.5) that

$$
\frac{1}{6} \frac{\mathrm{d}}{\mathrm{d} t} J^{6}+\frac{5}{9} \kappa_{v} \bar{J}^{6} \leq C\left\|\nabla \Phi_{s}\right\|_{L_{x, y}^{3 / 2}} J^{2} \bar{J}^{3}+C \mathbf{S} J^{2} \bar{J}^{3},
$$

which, by Young's inequality, implies that

$$
\frac{\mathrm{d}}{\mathrm{d} t} J^{6}+\kappa_{\boldsymbol{v}} \bar{J}^{6} \leq C\left\|\nabla \Phi_{s}\right\|_{L_{x, y}^{3 / 2}}^{2} J^{4}+C \mathbf{S}^{2} J^{4}
$$

We now set

$$
K(t)=\left(\sum_{k=1}^{2}\left\|\partial_{p} u_{k}(\cdot, t)\right\|_{L^{2}}^{2}\right)^{1 / 2}
$$

and

$$
\begin{array}{r}
\bar{K}(t)=\left(\sum_{k=1}^{2} \int_{\mathcal{M}}\left|\nabla\left(\partial_{p} u_{k}\right)\right|^{2} \mathrm{~d} \mathcal{M}+\sum_{k=1}^{2} \int_{\mathcal{M}}\left(\frac{g p}{R \bar{T}}\right)^{2}\left|\partial_{p p}\left(u_{k}\right)\right|^{2} \mathrm{~d} \mathcal{M}\right. \\
\left.+\frac{\mu_{\boldsymbol{v}}}{\nu_{\boldsymbol{v}}} \sum_{k=1}^{2} \int_{\Gamma_{i}}\left|\nabla u_{k}\right|^{2} \mathrm{~d} \Gamma_{i}\right)^{1 / 2} .
\end{array}
$$

In order to obtain the estimates for $K$ and $\bar{K}$, we multiply (A.4) by $-\partial_{p p} u_{k}$, where $k=1,2$ integrate over $\mathcal{M}$, and sum the resulting equations together; we find

$$
\begin{array}{r}
\frac{1}{2} \frac{\mathrm{d}}{\mathrm{d} t} K^{2}+\kappa_{\boldsymbol{v}} \bar{K}^{2}=\sum_{j, k=1}^{2} \int_{\mathcal{M}} u_{j} \partial_{j} u_{k} \partial_{p p} u_{k} \mathrm{~d} \mathcal{M}+\sum_{k=1}^{2} \int_{\mathcal{M}} u_{3} \partial_{p} u_{k} \partial_{p p} u_{k} \mathrm{~d} \mathcal{M} \\
+\sum_{k=1}^{2} \int_{\mathcal{M}} \partial_{k} \Phi_{s} \partial_{p p} u_{k} \mathrm{~d} \mathcal{M}-\sum_{k=1}^{2} \int_{\mathcal{M}} S_{k} \partial_{p p} u_{k} \mathrm{~d} \mathcal{M} .
\end{array}
$$


Integrations by parts on the first two terms on the right-hand side of (A.8) yield

$$
\begin{aligned}
\sum_{j, k=1}^{2} \int_{\mathcal{M}} u_{j} \partial_{j} u_{k} \partial_{p p} u_{k} \mathrm{~d} \mathcal{M}= & -\sum_{j, k=1}^{2} \int_{\mathcal{M}} \partial_{p} u_{j} \partial_{j} u_{k} \partial_{p} u_{k} \mathrm{~d} \mathcal{M}-\frac{\alpha_{\boldsymbol{v}}}{\nu_{\boldsymbol{v}}} \sum_{j, k=1}^{2} \int_{\Gamma_{i}} u_{j} \partial_{j} u_{k} u_{k} \mathrm{~d} \Gamma_{i} \\
+\frac{1}{2} \sum_{j, k=1}^{2} \int_{\mathcal{M}} \partial_{j} u_{j} \partial_{p} u_{k} \partial_{p} u_{k} \mathrm{~d} \mathcal{M} & =\sum_{j, k=1}^{2} \int_{\mathcal{M}} \partial_{p j} u_{j} u_{k} \partial_{p} u_{k} \mathrm{~d} \mathcal{M}+\sum_{j, k=1}^{2} \int_{\mathcal{M}} \partial_{p} u_{j} u_{k} \partial_{p j} u_{k} \mathrm{~d} \mathcal{M} \\
& +\frac{\alpha_{\boldsymbol{v}}}{2 \nu_{\boldsymbol{v}}} \sum_{j, k=1}^{2} \int_{\Gamma_{i}} \partial_{j} u_{j} u_{k} u_{k} \mathrm{~d} \Gamma_{i}-\sum_{j, k=1}^{2} \int_{\mathcal{M}} u_{j} \partial_{p j} u_{k} \partial_{p} u_{k} \mathrm{~d} \mathcal{M},
\end{aligned}
$$

and with the divergence free condition on $\boldsymbol{u}$, we have

$$
\begin{array}{r}
\sum_{k=1}^{2} \int_{\mathcal{M}} u_{3} \partial_{p} u_{k} \partial_{p p} u_{k} \mathrm{~d} \mathcal{M}=-\frac{1}{2} \sum_{k=1}^{2} \int_{\mathcal{M}} \partial_{p} u_{3} \partial_{p} u_{k} \partial_{p} u_{k} \mathrm{~d} \mathcal{M} \\
=\frac{1}{2} \sum_{j, k=1}^{2} \int_{\mathcal{M}} \partial_{j} u_{j} \partial_{p} u_{k} \partial_{p} u_{k} \mathrm{~d} \mathcal{M}=-\sum_{j, k=1}^{2} \int_{\mathcal{M}} u_{j} \partial_{p j} u_{k} \partial_{p} u_{k} \mathrm{~d} \mathcal{M} .
\end{array}
$$

The right-hand side of (A.8) is now less than or equal to

$$
\begin{array}{r}
C \sum_{j, k=1}^{2}\left\|u_{k}\right\|_{L^{6}}\left\|\partial_{p} u_{k}\right\|_{L^{3}}\left\|\nabla\left(\partial_{p} u_{j}\right)\right\|_{L^{2}}+C \sum_{j, k=1}^{2}\left\|u_{k}\right\|_{L^{6}}\left\|\partial_{p} u_{j}\right\|_{L^{3}}\left\|\nabla\left(\partial_{p} u_{k}\right)\right\|_{L^{2}} \\
+C \sum_{j, k=1}^{2}\left\|u_{j}\right\|_{L^{6}}\left\|\partial_{p} u_{k}\right\|_{L^{3}}\left\|\nabla\left(\partial_{p} u_{k}\right)\right\|_{L^{2}}+\sum_{k=1}^{2}\left\|S_{k}\right\|_{L^{2}}\left\|\partial_{p p} u_{k}\right\|_{L^{2}} \\
+\sum_{k=1}^{2}\left|\int_{\mathcal{M}} \partial_{k} \Phi_{s} \partial_{p p} u_{k} \mathrm{~d} \mathcal{M}\right|+\frac{\alpha_{\boldsymbol{v}}}{2 \nu_{\boldsymbol{v}}} \sum_{j, k=1}^{2}\left|\int_{\Gamma_{i}} \partial_{j} u_{j} u_{k} u_{k} \mathrm{~d} \Gamma_{i}\right| .
\end{array}
$$

The first three terms in (A.9) are bounded by $C J K^{1 / 2} \bar{K}^{3 / 2}$ and the fourth term is bounded by $C \mathbf{S} \bar{K}$. We rewrite the fifth term in (A.9) as

$$
\begin{aligned}
& \sum_{k=1}^{2}\left|\int_{\mathcal{M}^{\prime}} \partial_{k} \Phi_{s} \int_{p_{0}}^{p_{1}} \partial_{p p} u_{k} \mathrm{~d} p \mathrm{~d} \mathcal{M}^{\prime}\right|=\sum_{k=1}^{2}\left|\int_{\mathcal{M}^{\prime}} \partial_{k} \Phi_{s}\left(\partial_{p} u_{k}\right)\right|_{p=p_{0}}^{p=p_{1}} \mathrm{~d} \mathcal{M}^{\prime} \mid \\
= & \frac{\alpha_{\boldsymbol{v}}}{\nu_{\boldsymbol{v}}} \sum_{k=1}^{2}\left|\int_{\mathcal{M}^{\prime}} \partial_{k} \Phi_{s} u_{k}\right|_{p=p_{1}} \mathrm{~d} \mathcal{M}^{\prime}\left|\leq \frac{\alpha_{\boldsymbol{v}}}{\nu_{\boldsymbol{v}}} \sum_{k=1}^{2}\left\|\partial_{k} \Phi_{s}\right\|_{L_{x, y}^{3 / 2}}\left\|\left.u_{k}\right|_{p=p_{1}}\right\|_{L_{x, y}^{3}},\right.
\end{aligned}
$$

and we have by the trace theorem

$$
\left.\left.\left\|\left.u_{k}\right|_{p=p_{1}}\right\|\left\|_{L_{x, y}^{3}} \leq C\right\| u_{k}\right|_{p=p_{1}}\left\|_{L_{x, y}^{4}} \leq C\right\| u_{k}\right|_{p=p_{1}}\left\|_{H_{x, y}^{1 / 2}} \leq C\right\| u_{k} \|_{H^{1}(\mathcal{M})} \leq C \bar{E} .
$$

where

$$
\bar{E}(t)=\|\boldsymbol{v}(\cdot, t)\|_{H^{1}(\mathcal{M})}=\left(\sum_{k=1}^{2}\left\|u_{k}(\cdot, t)\right\|_{H^{1}(\mathcal{M})}^{2}\right)^{1 / 2}
$$


By Hölder's and Young's inequalities, the last term in (A.9) is estimated by

$$
C \sum_{j, k=1}^{2}\left\|\nabla u_{j}\right\|_{L^{2}\left(\Gamma_{i}\right)}\left\|u_{k}^{2}\right\|_{L^{3}\left(\Gamma_{i}\right)} \leq \frac{\kappa_{\boldsymbol{v}}}{8} \bar{K}^{2}+\frac{\kappa_{\boldsymbol{v}}}{8} \bar{J}^{6}+C .
$$

Now, we can deduce from (A.8) that

$$
\frac{1}{2} \frac{\mathrm{d}}{\mathrm{d} t} K^{2}+\kappa_{\boldsymbol{v}} \bar{K}^{2} \leq C J K^{1 / 2} \bar{K}^{3 / 2}+C\left\|\nabla \Phi_{s}\right\|_{L_{x, y}^{3 / 2}} \bar{E}+C \mathbf{S} \bar{K}+\frac{\kappa_{\boldsymbol{v}}}{8} \bar{K}^{2}+\frac{\kappa_{\boldsymbol{v}}}{8} \bar{J}^{6}+C,
$$

whence by noticing that $K(t) \leq \bar{E}(t)$,

$$
\frac{\mathrm{d}}{\mathrm{d} t} K^{2}+\kappa_{\boldsymbol{v}} \bar{K}^{2} \leq C \bar{E}^{2} J^{4}+C\left\|\nabla \Phi_{s}\right\|_{L_{x, y}^{3 / 2}}^{2}+\bar{E}^{2}+C \mathbf{S}^{2}+\frac{\kappa_{\boldsymbol{v}}}{8} \bar{J}^{6}+C .
$$

Next, we need an estimate on $\left\|\nabla \Phi_{s}\right\|_{L_{t}^{2} L_{x, y}^{3 / 2}}=\left\|\nabla \Phi_{s}\right\|_{L_{t}^{2} L_{x, y}^{3 / 2}\left(\left[\tau_{1}, \tau_{2}\right] \times \mathcal{M}^{\prime}\right)}$. For this purpose, we average the primitive equations in the vertical direction to obtain

$$
\begin{aligned}
\frac{\partial M u_{k}}{\partial t}-\mu_{\boldsymbol{v}} \Delta M u_{k}+\partial_{k} \Phi_{s}=- & \left.\frac{\alpha_{\boldsymbol{v}}}{p_{1}-p_{0}}\left(\frac{g p_{1}}{R \bar{T}}\right)^{2} u_{k}\right|_{p=p_{1}}, \\
& -\sum_{k=1}^{2} M\left(\partial_{j}\left(u_{j} u_{k}\right)\right)+M S_{k}, \quad k=1,2, \\
\partial_{1} M u_{1}+\partial_{2} M u_{2}=0 . &
\end{aligned}
$$

This is a linear two-dimensional Stokes problem for $\left(M \boldsymbol{v}, \Phi_{s}\right)$ with initial datum

$$
\left.M \boldsymbol{v}(\cdot, t)\right|_{t=\tau_{1}}=M \boldsymbol{v}\left(\cdot, \tau_{1}\right) .
$$

By the $L_{t}^{q} L_{x, y}^{p}$ estimates for the Stokes problem due to Sohr and von Wahl [34], we have

$$
\begin{array}{r}
\left\|\nabla \Phi_{s}\right\|_{L_{t}^{2} L_{x, y}^{3 / 2}} \leq C\left\|\left.u_{k}\right|_{p=p_{1}}\right\|_{L_{t}^{2} L_{x, y}^{3 / 2}}+C \sum_{j, k=1}^{2}\left\|M\left(\partial_{j}\left(u_{j} u_{k}\right)\right)\right\|_{L_{t}^{2} L_{x, y}^{3 / 2}} \\
+C \sum_{k=1}^{2}\left\|M S_{k}\right\|_{L_{t}^{2} L_{x, y}^{3 / 2}}+C \sum_{k=1}^{2}\left\|\nabla u_{k}\left(\cdot, \tau_{1}\right)\right\|_{L^{2}} \\
\leq C\left\|\left.u_{k}\right|_{p=p_{1}}\right\|_{L_{t}^{2} L_{x, y}^{3 / 2}}+C \sum_{j, k=1}^{2}\left\|u_{j} \partial_{j} u_{k}\right\|_{L_{t}^{2} L^{3 / 2}(\mathcal{M})} \\
+C \sum_{k=1}^{2}\left\|S_{k}\right\|_{L_{t}^{2} L^{2}(\mathcal{M})}+C\left\|\nabla \boldsymbol{v}\left(\cdot, \tau_{1}\right)\right\|_{L^{2}(\mathcal{M})}=: I_{1}+I_{2}+I_{3}+I_{4} .
\end{array}
$$

As before, by the trace theorem, the first term $I_{1}$ is estimated as

$$
I_{1} \leq C\left\|\left.\boldsymbol{v}\right|_{p=p_{1}}\right\|_{L_{t}^{2} L_{x, y}^{4}} \leq C\|\| \boldsymbol{v}\left\|_{H^{1}(\mathcal{M})}\right\|_{L_{t}^{2}} \leq C\|\bar{E}\|_{L_{t}^{2}}
$$

The second term $I_{2}$ is estimated as

$$
I_{2} \leq C \sum_{j, k=1}^{2}\|\| u_{j}\left\|_{L^{6}}\right\| \partial_{j} u_{k}\left\|_{L^{2}}\right\|_{L_{t}^{2}} \leq C\|J \bar{E}\|_{L_{t}^{2}}
$$


Collecting the estimates for $I_{1}$ and $I_{2}$, we obtain

$$
\left\|\nabla \Phi_{s}\right\|_{L_{t}^{2} L_{x, y}^{3 / 2}}^{2} \leq C\|\bar{E}\|_{L_{t}^{2}}^{2}+C\|J \bar{E}\|_{L_{t}^{2}}^{2}+C\|\mathbf{S}\|_{L_{t}^{2}}^{2}+C\left\|\nabla \boldsymbol{v}\left(\cdot, \tau_{1}\right)\right\|_{L^{2}}^{2} .
$$

Now, considering $0 \leq \tau_{1}<\tau_{2}<\tau_{3}$ and integrating (A.7) on $\left[\tau_{1}, \tau_{2}\right]$ yields

$$
J\left(\tau_{2}\right)^{6}+\kappa_{\boldsymbol{v}}\left\|\bar{J}^{3}\right\|_{L_{t}^{2}\left(\tau_{1}, \tau_{2}\right)}^{2} \leq J\left(\tau_{1}\right)^{6}+\left\|\nabla \Phi_{s}\right\|_{L_{t}^{2} L_{x, y}^{3 / 2}}^{2} \sup _{\tau_{1} \leq \tau \leq \tau_{3}} J(\tau)^{4}+C\|\mathbf{S}\|_{L_{t}^{2}}^{2} \sup _{\tau_{1} \leq \tau \leq \tau_{3}} J(\tau)^{4},
$$

where the $L_{t}^{2}$-norms are taken over $\left[\tau_{1}, \tau_{3}\right]$. Implementing the pressure estimate (A.13), we obtain

$$
\begin{array}{r}
J\left(\tau_{2}\right)^{6}+\kappa_{\boldsymbol{v}}\left\|\bar{J}^{3}\right\|_{L_{t}^{2}\left(\tau_{1}, \tau_{2}\right)}^{2} \leq J\left(\tau_{1}\right)^{6}+C\|\bar{E}\|_{L_{t}^{2}}^{2} \sup _{\tau_{1} \leq \tau \leq \tau_{3}} J(\tau)^{4}+C\|\bar{E}\|_{L_{t}^{2}}^{2} \sup _{\tau_{1} \leq \tau \leq \tau_{3}} J(\tau)^{6} \\
+C\left(\|\mathbf{S}\|_{L_{t}^{2}}^{2}+\left\|\nabla \boldsymbol{v}\left(\cdot, \tau_{1}\right)\right\|_{L^{2}}^{2}\right) \sup _{\tau_{1} \leq \tau \leq \tau_{3}} J(\tau)^{4},
\end{array}
$$

which is valid for all $\tau_{2} \in\left[\tau_{1}, \tau_{3}\right]$. Hence, taking $\sup _{\tau_{1} \leq \tau \leq \tau_{3}}$ on both sides and using the Cauchy-Schwarz inequality to absorb the terms involving $\sup _{\tau_{1} \leq \tau \leq \tau_{3}} J(\tau)^{4}$ into the left-hand side, we obtain

$$
\begin{array}{r}
\sup _{\tau_{1} \leq \tau \leq \tau_{3}} J(\tau)^{6}+\kappa_{\boldsymbol{v}}\left\|\bar{J}^{3}\right\|_{L_{t}^{2}\left(\tau_{1}, \tau_{3}\right)}^{2} \leq 2 J\left(\tau_{1}\right)^{6}+C\|\bar{E}\|_{L_{t}^{2}}^{4}+C\|\bar{E}\|_{L_{t}^{2}}^{2} \sup _{\tau_{1} \leq \tau \leq \tau_{3}} J(\tau)^{6} \\
+C\left(\|\mathbf{S}\|_{L_{t}^{2}}^{4}+\left\|\nabla \boldsymbol{v}\left(\cdot, \tau_{1}\right)\right\|_{L^{2}}^{4}\right) .
\end{array}
$$

Similarly, we integrate (A.11) on $\left[\tau_{1}, \tau_{2}\right]$ and use the pressure estimate (A.13) to find

$$
\begin{aligned}
K\left(\tau_{2}\right)^{2} & +\kappa_{\boldsymbol{v}}\|\bar{K}\|_{L_{t}^{2}\left(\tau_{1}, \tau_{2}\right)}^{2} \leq K\left(\tau_{1}\right)^{2}+C\|\bar{E}\|_{L_{t}^{2}}^{2} \sup _{\tau_{1} \leq \tau \leq \tau_{3}} J(\tau)^{4}+C\|\bar{E}\|_{L_{t}^{2}}^{2} \\
& +C\|\bar{E}\|_{L_{t}^{2}}^{2} \sup _{\tau_{1} \leq \tau \leq \tau_{3}} J(\tau)^{2}+\frac{\kappa_{\boldsymbol{v}}}{8}\left\|\bar{J}^{3}\right\|_{L_{t}^{2}}^{2}+C\left(\|\mathbf{S}\|_{L_{t}^{2}}^{2}+\left\|\nabla \boldsymbol{v}\left(\cdot, \tau_{1}\right)\right\|_{L^{2}}^{2}+1\right) .
\end{aligned}
$$

Therefore,

$$
\begin{array}{rl}
\sup _{\tau_{1} \leq \tau \leq \tau_{3}} & K(\tau)^{2}+\kappa_{\boldsymbol{v}}\|\bar{K}\|_{L_{t}^{2}\left(\tau_{1}, \tau_{3}\right)}^{2} \leq K\left(\tau_{1}\right)^{2}+C\|\bar{E}\|_{L_{t}^{2}}^{2} \sup _{\tau_{1} \leq \tau \leq \tau_{3}} J(\tau)^{4}+C\|\bar{E}\|_{L_{t}^{2}}^{2} \\
& +C\|\bar{E}\|_{L_{t}^{2}}^{2} \sup _{\tau_{1} \leq \tau \leq \tau_{3}} J(\tau)^{2}+\frac{\kappa_{\boldsymbol{v}}}{8}\left\|\bar{J}^{3}\right\|_{L_{t}^{2}}^{2}+C\left(\|\mathbf{S}\|_{L_{t}^{2}}^{2}+\left\|\nabla \boldsymbol{v}\left(\cdot, \tau_{1}\right)\right\|_{L^{2}}^{2}+1\right) .
\end{array}
$$

Now, choose $\delta>0$ such that

$$
\|\bar{E}\|_{L_{t}^{2}(t, t+2 \delta)}^{2} \leq \frac{1}{\gamma}, \quad \forall t \in\left[0, t_{1}\right],
$$

where $\gamma$ is a sufficiently large constant to be determined later. Let $t^{0}=0$ and then for $j=1, \cdots, l$, choose $t^{j} \in(j \delta,(j+1) \delta)$ such that

$$
\left\|\boldsymbol{v}\left(\cdot, t^{j}\right)\right\|_{H^{1}(\mathcal{M})}^{2} \leq \frac{1}{\delta} \int_{j \delta}^{(j+1) \delta}\|\boldsymbol{v}(\cdot, \tau)\|_{H^{1}(\mathcal{M})}^{2} \mathrm{~d} \tau \leq \frac{1}{\delta \gamma},
$$

where $l$ is the largest integer such that $(l+1) \delta \leq t_{1}$. Let $t^{l+1}=t_{1}$. Note that

$$
t^{j+1}-t^{j} \leq 2 \delta, \quad \forall j=0, \ldots, l .
$$


Summing the inequalities (A.14) and (A.15) with $\tau_{1}=t^{j}$ and $\tau_{3}=t^{j+1}$ implies that

$$
\begin{array}{r}
\sup _{t^{j} \leq t \leq t^{j+1}} J(t)^{6}+\frac{7 \kappa_{\boldsymbol{v}}}{8}\left\|\bar{J}^{3}\right\|_{L_{t}^{2}}^{2}+\sup _{t^{j} \leq t \leq t^{j+1}} K(t)^{2}+\kappa_{\boldsymbol{v}}\|\bar{K}\|_{L_{t}^{2}}^{2} \leq 2 J\left(t^{j}\right)^{6}+K\left(t^{j}\right)^{2}+C\|\bar{E}\|_{L_{t}^{2}}^{4} \\
+C\|\bar{E}\|_{L_{t}^{2}}^{2} \sup _{\tau_{1} \leq \tau \leq \tau_{3}} J(\tau)^{6}+C\|\bar{E}\|_{L_{t}^{2}}^{2} \sup _{\tau_{1} \leq \tau \leq \tau_{3}} J(\tau)^{4}+C\|\bar{E}\|_{L_{t}^{2}}^{2}+C\|\bar{E}\|_{L_{t}^{2}}^{2} \sup _{\tau_{1} \leq \tau \leq \tau_{3}} J(\tau)^{2} \\
+C\left(\|\mathbf{S}\|_{L_{t}^{2}}^{4}+\left\|\nabla \boldsymbol{v}\left(\cdot, t^{j}\right)\right\|_{L^{2}}^{4}\right)+C\left(\|\mathbf{S}\|_{L_{t}^{2}}^{2}+\left\|\nabla \boldsymbol{v}\left(\cdot, t^{j}\right)\right\|_{L^{2}}^{2}+1\right),
\end{array}
$$

which, by using Young's inequality and absorbing $\sup _{\tau_{1} \leq \tau \leq \tau_{3}} J(\tau)^{4}$ and $\sup _{\tau_{1} \leq \tau \leq \tau_{3}} J(\tau)^{2}$ into the left-hand side, yields

$$
\begin{aligned}
\sup _{t^{j} \leq t \leq t^{j+1}} J(t)^{6}+ & \sup _{t^{j} \leq t \leq t^{j+1}} K(t)^{2}+\kappa_{\boldsymbol{v}}\|\bar{K}\|_{L_{t}^{2}}^{2} \leq 4 J\left(t^{j}\right)^{6}+2 K\left(t^{j}\right)^{2}+C\|\bar{E}\|_{L_{t}^{2}}^{4} \\
& +C\|\bar{E}\|_{L_{t}^{2}}^{2} \sup _{\tau_{1} \leq \tau \leq \tau_{3}} J(\tau)^{6}+C\left(\|\mathbf{S}\|_{L_{t}^{2}}^{4}+\left\|\nabla \boldsymbol{v}\left(\cdot, t^{j}\right)\right\|_{L^{2}}^{4}+1\right) .
\end{aligned}
$$

Using (A.16) and (A.17) and

$$
\max \left\{J\left(t^{j}\right)^{2}, K\left(t^{j}\right)^{2}\right\} \leq C \bar{E}\left(t^{j}\right)^{2}=C\left\|\boldsymbol{v}\left(\cdot, t^{j}\right)\right\|_{H^{1}(\mathcal{M})}^{2} \leq \frac{C}{\delta \gamma}
$$

we obtain

$$
\begin{array}{rl}
\sup _{t^{j} \leq t \leq t^{j+1}} & J(t)^{6}+\sup _{t^{j} \leq t \leq t^{j+1}} K(t)^{2}+\kappa_{\boldsymbol{v}}\|\bar{K}\|_{L_{t}^{2}}^{2} \leq \frac{C}{\delta^{3} \gamma^{3}}+\frac{C}{\delta \gamma} \\
+\frac{C}{\gamma^{2}}+\frac{C}{\gamma} \sup _{\tau_{1} \leq \tau \leq \tau_{3}} J(\tau)^{6}+C\left(\|\mathbf{S}\|_{L_{t}^{2}}^{4}+\frac{C}{\delta^{2} \gamma^{2}}+1\right) .
\end{array}
$$

Therefore, choosing $\gamma>0$ large enough to absorb the fourth term in in the right-hand side of (A.19), we eventually find that

$$
J(t), K(t),\|\bar{K}\|_{L^{2}\left(0, t_{1}\right)} \leq \widetilde{M}, \quad t \in\left[0, t_{1}\right]
$$

where

$$
\widetilde{M}:=\left(\frac{C}{\delta^{3} \gamma^{3}}+\frac{C}{\delta \gamma}+\frac{C}{\gamma^{2}}+C\left(\|\mathbf{S}\|_{L_{t}^{2}}^{4}+\frac{C}{\delta^{2} \gamma^{2}}+1\right)\right)\left(\frac{1}{\kappa_{\boldsymbol{v}}}+1\right) .
$$

We are now in position to bound $\|\boldsymbol{v}(\cdot, t)\|_{\mathbf{v}}$ for all $t \in\left[0, t_{1}\right]$ with a constant independent of $t_{1}$. Taking the inner product of each side of (A.1) with $A_{\boldsymbol{v}} \boldsymbol{v}$ leads to

$$
\begin{aligned}
\frac{1}{2} \frac{\mathrm{d}}{\mathrm{d} t}\left\|A_{\boldsymbol{v}}^{1 / 2} \boldsymbol{v}\right\|_{L^{2}}^{2}+\left\|A_{\boldsymbol{v}} \boldsymbol{v}\right\|_{L^{2}}^{2} \leq & \sum_{k=1}^{2}\left\|u_{j} \partial_{j} \boldsymbol{v}\right\|_{L^{2}}\left\|A_{\boldsymbol{v}} \boldsymbol{v}\right\|_{L^{2}}+\left\|u_{3} \partial_{p} \boldsymbol{v}\right\|_{L^{2}}\left\|A_{\boldsymbol{v}} \boldsymbol{v}\right\|_{L^{2}} \\
& +\left\|S_{\boldsymbol{v}}\right\|_{L^{2}}\left\|A_{\boldsymbol{v}} \boldsymbol{v}\right\|_{L^{2}}
\end{aligned}
$$

which implies that

$$
\begin{aligned}
\frac{\mathrm{d}}{\mathrm{d} t}\left\|A_{\boldsymbol{v}}^{1 / 2} \boldsymbol{v}\right\|_{L^{2}}^{2} & +\left\|A_{\boldsymbol{v}} \boldsymbol{v}\right\|_{L^{2}}^{2} \leq \sum_{k=1}^{2}\left\|u_{j} \partial_{j} \boldsymbol{v}\right\|_{L^{2}}^{2}+\left\|u_{3} \partial_{p} \boldsymbol{v}\right\|_{L^{2}}^{2}+C\left\|S_{\boldsymbol{v}}\right\|_{L^{2}}^{2} \\
& \leq C J^{2}\left\|A_{\boldsymbol{v}}^{1 / 2} \boldsymbol{v}\right\|_{L^{2}}\left\|A_{\boldsymbol{v}} \boldsymbol{v}\right\|_{L^{2}}+C K \bar{K}\left\|A_{\boldsymbol{v}}^{1 / 2} \boldsymbol{v}\right\|_{L^{2}}\left\|A_{\boldsymbol{v}} \boldsymbol{v}\right\|_{L^{2}}+C\left\|S_{\boldsymbol{v}}\right\|_{L^{2}}^{2},
\end{aligned}
$$

where we used the anisotropic estimates for the second term in the right-hand side. Therefore,

$$
\frac{\mathrm{d}}{\mathrm{d} t}\left\|A_{\boldsymbol{v}}^{1 / 2} \boldsymbol{v}\right\|_{L^{2}}^{2}+\left\|A_{\boldsymbol{v}} \boldsymbol{v}\right\|_{L^{2}}^{2} \leq C J^{4}\left\|A_{\boldsymbol{v}}^{1 / 2} \boldsymbol{v}\right\|_{L^{2}}^{2}+C K^{2} \bar{K}^{2}\left\|A_{\boldsymbol{v}}^{1 / 2} \boldsymbol{v}\right\|_{L^{2}}^{2}+C\left\|S_{\boldsymbol{v}}\right\|_{L^{2}}^{2},
$$


and the uniform bound of $\|\boldsymbol{v}(t)\|_{\mathbf{V}}$ follows from the Gronwall lemma and the estimate (A.20). Hence, we proved the global existence of Theorem A.1. The uniqueness follows similarly as in [20, pp. 2748] and since it is not important for our goal here, we thus omit the details. This ends the proof of Theorem A.1.

\section{ACKNOWLEDGMENTS}

MCZ thanks Professor C.G. Gal for suggesting the use of the De Giorgi technique for the maximum principle results in Section 8. MCZ, AH, and RT were partially supported by the National Science Foundation under the grant NSF DMS-1206438 and by the Research Fund of Indiana University, IK was supported in part by the NSF grant DMS-1311943, while MZ was supported in part by the NSF grant DMS-1109562.

\section{REFERENCES}

[1] P. Azérad and F. Guillén, Mathematical justification of the hydrostatic approximation in the primitive equations of geophysical fluid dynamics, SIAM J. Math. Anal. 33 (2001), 847-859.

[2] V. Barbu, Nonlinear differential equations of monotone types in Banach spaces, Springer, New York, 2010 .

[3] A. Bousquet, M. Coti Zelati, and R. Temam, Phase transition models in atmospheric dynamics, Milan Journal of Mathematics 82 (2014), 99-128.

[4] C. Cao and E. S. Titi, Global well-posedness of the three-dimensional viscous primitive equations of large scale ocean and atmosphere dynamics, Ann. of Math. (2) 166 (2007), 245-267.

[5] M. Coti Zelati, M. Frémond, R. Temam, and J. Tribbia, The equations of the atmosphere with humidity and saturation: uniqueness and physical bounds, Phys. D 264 (2013), 49-65.

[6] M. Coti Zelati and R. Temam, The atmospheric equation of water vapor with saturation, Boll. Unione Mat. Ital. (9) 5 (2012), 309-336.

[7] E. De Giorgi, Sulla differenziabilità e l'analiticità delle estremali degli integrali multipli regolari, Mem. Accad. Sci. Torino. Cl. Sci. Fis. Mat. Nat. (3) 3 (1957), 25-43 (Italian).

[8] I. Ekeland and R. Temam, Convex analysis and variational problems, North-Holland Publishing Co., Amsterdam, 1976. Translated from the French; Studies in Mathematics and its Applications, Vol. 1.

[9] B. D. Ewald and R. Temam, Maximum principles for the primitive equations of the atmosphere, Discrete Contin. Dynam. Systems 7 (2001), 343-362.

[10] A. E. Gill, Atmosphere-ocean dynamics, International Geophysics Series, Vol. 30, Academic Press, San Diego, 1982.

[11] F. Guillén-González, N. Masmoudi, and M. A. Rodríguez-Bellido, Anisotropic estimates and strong solutions of the primitive equations, Differential Integral Equations 14 (2001), 1381-1408.

[12] B. Guo and D. Huang, Existence of weak solutions and trajectory attractors for the moist atmospheric equations in geophysics, J. Math. Phys. 47 (2006), 083508, 23.

[13] Existence of the universal attractor for the 3-D viscous primitive equations of large-scale moist atmosphere, J. Differential Equations 251 (2011), 457-491.

[14] G. J. Haltiner, Numerical weather prediction, John Wiley \& Sons, New York, 1971.

[15] G. J. Haltiner and R. T. Williams, Numerical prediction and dynamic meteorology, John Wiley \& Sons, New York, 1980.

[16] D. Kinderlehrer and G. Stampacchia, An introduction to variational inequalities and their applications, Academic Press Inc., New York, 1980.

[17] G. M. Kobelkov, Existence of a solution 'in the large' for the 3D large-scale ocean dynamics equations, C. R. Math. Acad. Sci. Paris 343 (2006), 283-286 (English, with English and French summaries).

[18] G. M. Kobelkov, Existence of a solution "in the large" for ocean dynamics equations, J. Math. Fluid Mech. 9 (2007), 588-610.

[19] I. Kukavica, Y. Pei, W. Rusin, and M. Ziane, Primitive equations with continuous initial data, Nonlinearity 27 (2014), 1135-1155.

[20] I. Kukavica and M. Ziane, On the regularity of the primitive equations of the ocean, Nonlinearity 20 (2007), 2739-2753. 
[21] Uniform gradient bounds for the primitive equations of the ocean, Differential Integral Equations 21 (2008), 837-849.

[22] J.-L. Lions, Quelques méthodes de résolution des problèmes aux limites non linéaires, Dunod, 1969.

[23] J.-L. Lions, R. Temam, and S. Wang, New formulations of the primitive equations of atmosphere and applications, Nonlinearity 5 (1992), 237-288.

[24] J.-L. Lions, R. Temam, and S. H. Wang, On the equations of the large-scale ocean, Nonlinearity 5 (1992), 1007-1053.

[25] J.-L. Lions, R. Temam, and S. Wang, Models for the coupled atmosphere and ocean. (CAO I,II), Comput. Mech. Adv. 1 (1993), 5-119.

[26] J.-L. Lions, R. Temam, and S. H. Wang, Mathematical theory for the coupled atmosphere-ocean models. (CAO III), J. Math. Pures Appl. (9) 74 (1995), 105-163.

[27] A. Miranville and R. Temam, Mathematical modeling in continuum mechanics, Cambridge University Press, Cambridge, 2005.

[28] J. Pedlosky, Geophysical Fluid Dynamics, Springer-Verlag, New York, 1987.

[29] M. Petcu, R. M. Temam, and M. Ziane, Some mathematical problems in geophysical fluid dynamics, Handbook of numerical analysis. Vol. XIV. Special volume: computational methods for the atmosphere and the oceans, Vol. 14, Elsevier/North-Holland, Amsterdam, 2009, pp. 577-750.

[30] L. F. Richardson, Weather prediction by numerical process, Cambridge Mathematical Library, Cambridge University Press, Cambridge, 2007.

[31] R. T. Rockafellar, Convex analysis, Princeton University Press, Princeton, NJ, 1997.

[32] R. R. Rogers and M. K. Yau, A Short Course in Cloud Physics, Butterworth-Heinemann, 1989.

[33] H. Sohr, The Navier-Stokes equations, Birkhäuser Verlag, Basel, 2001.

[34] H. Sohr and W. von Wahl, On the regularity of the pressure of weak solutions of Navier-Stokes equations, Arch. Math. (Basel) 46 (1986), 428-439.

[35] R. Temam, Navier-Stokes equations and nonlinear functional analysis, SIAM, Philadelphia, 1995.

[36] _ Infinite-dimensional dynamical systems in mechanics and physics, Springer-Verlag, New York, 1997.

[37] Navier-Stokes equations, theory and numerical analysis, AMS Chelsea Publishing, Providence, 2001.

[38] R. Temam and M. Ziane, Some mathematical problems in geophysical fluid dynamics, Handbook of mathematical fluid dynamics. Vol. III, North-Holland, Amsterdam, 2004, pp. 535-657.

[39] D. L. Turcotte and G. Schubert, Geodynamics: Applications of Continuum Physics to Geological Problems, John Wiley and Sons, New York, 1982.

Indiana University - Institute for Scientific Computing and Applied Mathematics

Rawles Hall, Bloomington, IN 47405, USA

E-mail address: micotize@indiana.edu (M. Coti Zelati)

E-mail address: aimhuang@indiana.edu (A. Huang)

E-mail address: temam@indiana.edu (R. Temam)

University of Southern California - Department of Mathematics

3620 S. Vermont Ave., KAP 108, Los Angeles, California 90089, USA

E-mail address: kukavica@usc.edu (I. Kukavica)

E-mail address: ziane@math.usc.edu (M. Ziane) 